\title{
A study of the large voids in the spatial distribution of galaxy clusters in the Northern Galactic Hemisphere
}

\author{
K.Y. Stavrev \\ Institute of Astronomy, Bulgarian Academy of Sciences, 72 Tsarigradsko Shosse Blvd., BG-1784 Sofia, Bulgaria \\ e-mail: kstavrev@skyarchive.org
}

Received July 22, 1999; accepted March 23, 2000

\begin{abstract}
We present a mapping of the large voids $(D \geq$ $\left.50 h^{-1} \mathrm{Mpc}\right)$ in the spatial distribution of clusters of galaxies in the Northern Galactic Hemisphere in a volume defined by galactic latitude $b \geq+30^{\circ}$ and redshift $z \leq 0.14$. Rich clusters with spectroscopically measured or photometrically estimated redshifts are used as tracers of the large-scale structure of the Universe. An automated void-search and analysis procedure which identifies a void as a system of intersecting empty spheres is applied, allowing for a description of the void dimensions, volumes and shapes more complete than in previous investigations. A number of void catalogues corresponding to different tracer types, different sources of redshift data, and different versions of the search method have been generated and analysed. Visualizations of the 2-D and 3-D distributions of voids are presented. The estimated mean dimensions of the voids of $R \geq 1$ and $R \geq 0$ Abell/ACO clusters are $D_{\mathrm{e}}=105.0 \pm 5.6 \bar{h}^{-1} \mathrm{Mpc}$ and $D_{\mathrm{e}}=87.2 \pm 4.1 h^{-1} \mathrm{Mpc}$, respectively, where $D_{\mathrm{e}}$ is the equivalent void diameter. We have identified the poor clusters, groups, and galaxies populating the voids of rich clusters and constructed radial density profiles of the voids, which show the presence of a void hierarchy.
\end{abstract}

Key words: cosmology: observations - large-scale structure of Universe - galaxies: clusters: general methods: data analysis

\section{Introduction}

The voids in the spatial distribution of galaxies and clusters of galaxies, discovered more than two decades ago (Tifft \& Gregory 1976; Gregory \& Thompson 1978; Jõeveer \& Einasto 1978; Kirshner et al. 1981; Bahcall \& Soneira 1982a, 1982b), are a dominant feature of the largescale structure (LSS) of the Universe. The observational

Send offprint requests to: K.Y. Stavrev studies of their properties (geometrical parameters, content, environment) are of great importance for testing basic assumptions in the contemporary theoretical models and scenarios of the formation and evolution of the LSS.

One major complication in void studies arises from the difficulty to obtain spatial distributions of the tracers of the LSS with spectroscopically measured redshifts which are fairly complete in volumes both wide and deep. From this point of view the use of rich clusters of galaxies as tracers allows to study the largest spatial volumes.

Wide-angle studies of the voids in the distribution of clusters of galaxies containing lists (catalogues) of the identified voids have been carried out so far by Batuski \& Burns (1985), Tully (1986), Stavrev (1990a, 1990b), and Einasto et al. (1994). All of them are based on Abell/ACO clusters (Abell 1958; Abell et al. 1989) as tracers. Since then, new redshift data for the clusters have been accumulated. An important step in this direction is the MX Northern Abell Cluster Redshift Survey (Slinglend et al. 1998), not only because of the increased number of clusters with measured redshifts, but also because of the reliable determination of the cluster redshifts excluding to a great extent the foreground/background contamination. The newly accumulated redshift data allows now for a more complete and correct mapping of the voids in the distribution of galaxy clusters.

Recent observational studies confirm a hierarchical structure of the voids, suggested from theoretical considerations for the void evolution by Dubinski et al. (1993), and van de Weygaert \& van Kampen (1993). According to Einasto et al. (1994) the voids determined by rich clusters of galaxies are divided into smaller voids by filaments of very poor clusters and galaxies. Lindner et al. (1995, 1996) find a void hierarchy in the Northern Local Supervoid (Einasto et al. 1983): voids defined by poor (Zwicky) clusters are divided into smaller voids by latetype galaxies. These voids contain fainter galaxies which define still smaller voids. The presence of the void hierarchy makes it reasonable to combine the observations of 
different types of tracers of the LSS - from rich clusters of galaxies down to poor clusters, groups, and single galaxies. The usefulness of this approach, however, is limited by the incompleteness of the samples of poor clusters, groups and galaxies with measured redshifts in volumes in which the rich clusters of galaxies form complete samples.

The choice of appropriate numerical techniques for void search and analysis is an important aspect of the void studies. The method of the largest empty sphere, nested in the void, has been most often used in searches for voids in the distribution of clusters. It has been first applied by Tully (1986), and later by Einasto et al. (1989) and Stavrev (1990a, 1990b). Tifft \& Gregory (1988) and Stavrev (1991) have proposed an improvement of this method through approximation of a void by a system of intersecting spheres (spheroids). Such an approach allows for a more complete description of the void dimensions, volumes and shapes. An application of this version of the method is given in Stavrev (1998). Recently similar multisphere techniques were developed by El-Ad et al. (1996), as well as by Aikio \& Mähönen (1998), and applied to the study of voids in the distribution of IRAS galaxies (ElAd et al. 1997) and southern sky galaxies (El-Ad \& Piran 1997).

The goals of this paper are (1) to map as completely as possible the large voids in the distribution of clusters of galaxies in the Northern Galactic Hemisphere out to medium-large distances $\left(\sim 400 h^{-1} \mathrm{Mpc}\right)$ combining redshift data from many sources, and using estimated redshifts, (2) to define void parameters on the basis of a more elaborate procedure for void search and analysis, (3) to obtain estimates of the mean characteristics of voids from a statistical analysis of homogeneous samples of voids, and (4) to identify the void population and study its distribution in the light of the concept of a void hierarchy. To achieve these goals we have developed and applied an automated system for void search and analysis.

The observational data used in this study are described in Sect. 2. The Automated Void Search and Analysis System is presented in Sect. 3, including the void-search algorithm and the void parameterization. Some functions of the system are explained also in Sects. 5 and 6. Section 4 presents the generated void catalogues, and Sect. 5 contains the results from their analysis, including an automated comparison of the catalogues obtained with different tracers, a comparison with voids known from previous investigations, and a statistical analysis. The void population and void shell are discussed in Sect. 6. Finally, Sect. 7 gives a summary of the results.

\section{Composition of the observational samples}

\subsection{Combining redshift data from different sources}

The redshift data used in the present study which is limited to the Northern Galactic Hemisphere (NGH) have been compiled from the following sources:

(1) A Compilation of Clusters of Galaxies with Published Redshifts, version 1996.0, by V.S. Lebedev and I.A. Lebedeva (a description of an earlier version is given in Lebedev \& Lebedeva 1991), with redshifts for 1500 clusters and groups (with at least 20 member galaxies) in the NGH, 859 of which are Abell/ACO (A/ACO) clusters.

(2) The NASA/IPAC Extragalactic Database (NED), version 1997 (see description in Madore et al. 1992), with redshifts for 968 clusters and 190 groups in the NGH, 759 of which are A/ACO clusters.

(3) A Catalogue of Measured Redshifts of Abell Clusters of Galaxies, version 1997 (Andernach \& Tago 1998; Andernach 1997), with 944 A/ACO clusters in the NGH.

(4) The MX Northern Abell Cluster Redshift Survey (Slinglend et al. 1998; Batuski et al. 1998), with redshifts for 62 clusters and 801 galaxies in the NGH.

(5) The Center for Astrophysics (CfA) Redshift Catalogue (ZCAT), version March 3, 1997 (see description in Huchra et al. 1992), containing redshifts for 21919 galaxies in the NGH.

For convenience, we shall use the following abbreviations for sources 1-5, respectively: LEB, NED, AND, MXS, CfA.

As can be seen, we use four compilations, based on hundreds of original sources, and one new redshift survey, not yet included in any compilation at the time of the present study. They reflect the status of the available redshift data for the clusters, groups, and galaxies in the beginning of 1998 .

The numbers of the A/ACO clusters given above include the supplementary southern clusters (ACO S) as well. Let us note that because of the limitation to the NGH (galactic latitude $b \geq 0^{\circ}$ ) the great majority $(>90 \%$ ) of the compiled $\mathrm{A} / \mathrm{ACO}$ clusters are northern Abell clusters (Abell 1958).

Besides A/ACO clusters the compilations LEB and NED contain also Zwicky clusters (Zwicky et al. 1961), other types of clusters like poor clusters (Morgan et al. 1975; Albert et al. 1977), clusters from the Shane-Wirtanen counts (Shectman 1985), distant clusters (Gunn et al. 1986), clusters from the Einstein Observatory Medium Sensitivity Survey (Gioia et al. 1990) and the ROSAT all-sky survey (Voges 1992). The groups of galaxies in LEB and NED are mainly nearby groups (Huchra \& Geller 1982), compact groups (Hickson 1982), groups in the two CfA redshift surveys (Geller \& Huchra 1983; Ramella et al. 1989), and groups in individual clusters and superclusters (e.g. Ciardullo et al. 1983; Hopp \& Materne 1985).

No indication which object is a group is given in LEB. We have found from the original data sources referenced in LEB that the number of groups in this compilation (in the NGH) is $\lesssim 100$. 
The preliminary examination of the spatial distribution of the different tracers from data sources 1-5 shows that only the A/ACO clusters can form complete enough samples in large volumes suitable for large void studies. The completeness limits for all other tracers (nonA/ACO clusters, groups, galaxies) do not go beyond $z \sim$ $0.03-0.04$. Therefore, we use in this study for void identification only distributions of A/ACO clusters. A limited application of the other tracers has been done only for investigating the substructure of voids in the distribution of rich A/ACO clusters (Sect. 6). (Processing of samples containing galaxies, as well as groups and poor clusters, in smaller and nearer spatial volumes in which the completeness of these objects is higher will be done in a separate study.)

The compilations LEB, NED, and AND are maintained independently by their authors. Furthermore, the compilation versions we use refer to somewhat different times of last update. Therefore, they do not overlap completely and each one of them contains objects which are not present in the other compilations. It follows that by merging them we can increase the total number of objects with spectroscopically measured redshifts.

We have first combined the data from LEB, NED, and MXS. The data from the new unpublished version of $\mathrm{AND}$, only for $\mathrm{A} / \mathrm{ACO}$ clusters missing in the other three sources, became available to us and was added later as a final enlargement of the data.

After a preliminary check of the data in LEB and NED we have removed from them several dozens of objects which are predominantly subcomponents of clusters with the same coordinates and redshift as the main component, or objects which are erroneously included in the compilations as spectroscopically observed, while, in fact, their redshifts are photometric estimates (Andernach 1997).

Before combining the data, the redshifts, when heliocentric $\left(z_{\mathrm{h}}\right)$, have been transformed to galactocentric $\left(z_{\mathrm{g}}\right)$ following

$z_{\mathrm{g}}=z_{\mathrm{h}}+\frac{300}{c} \sin l \cos b$,

where $l$ and $b$ are the galactic coordinates, and $c$ is the speed of light. The distance $r$ to the objects has been derived from the Hubble law assuming a Hubble constant $H_{0}=100 \mathrm{~km} \mathrm{~s}^{-1} \mathrm{Mpc}^{-1}$. The Cartesian coordinates $x, y, z$ of the objects have been calculated from their spherical coordinates $r, l, b$ with the transformation

$x=r \cos l \cos b$,

$y=r \sin l \cos b$,

$z=r \sin b$

in a coordinate system centred in the Galaxy with axes $x$ and $y$ directed to $l=0^{\circ}, b=0^{\circ}$ and $l=90^{\circ}, b=0^{\circ}$, respectively, and axis $z$ directed to the North Galactic Pole (NGP). In order to be able to construct the 2-D distribution of objects on the sky we have calculated also their

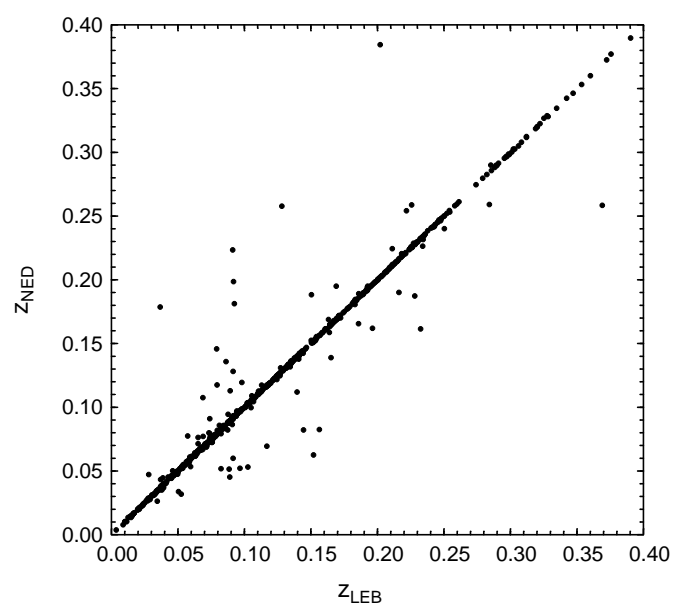

Fig. 1. Comparison of the redshifts of clusters and groups in the compilation of Lebedev and Lebedeva $\left(z_{\mathrm{LEB}}\right)$ with the redshifts in NED $\left(z_{\mathrm{NED}}\right)$ for galactic latitude $b \geq 0^{\circ}$

rectangular coordinates $x_{\mathrm{L}}, y_{\mathrm{L}}$ in an azimuthal equal-area projection (Lambert projection) from

$$
\begin{aligned}
A & =2 \sin \left(45^{\circ}-\frac{b}{2}\right), \\
x_{\mathrm{L}} & =A \cos l, \\
y_{\mathrm{L}} & =-A \sin l,
\end{aligned}
$$

where $b \geq 0^{\circ}$, for a coordinate system centred on the NGP and axes $x$ and $y$ directed respectively to $l=0^{\circ}, b=0^{\circ}$ and $l=270^{\circ}, b=0^{\circ}$.

The combination of the data from the different sources is complicated by the presence of discrepant redshifts for a part of the objects, as well as by the necessity to cross-identify correctly (automatically) the identical objects whose designations, coordinates, redshifts, etc. may not exactly coincide in the different sources.

The comparison of the LEB and NED compilations gives 800 coinciding objects whose redshifts are compared in Fig. 1 (for $z \leq 0.4$ ). As it is seen, most of the clusters and groups are situated along the line $z_{\mathrm{LEB}}=z_{\mathrm{NED}}$. However, for several dozens of objects $(\sim 10 \%)$ the differences $\Delta z=z_{\mathrm{LEB}}-z_{\mathrm{NED}}$ are significant. We have found that in many cases the large $\Delta z$ are an effect of the background contamination which splits clusters into two or more components at different distances along the line-ofsight.

The MX Survey contains the most reliable data for the cluster redshifts in comparison with the other three sources of data. This is due to the large mean number $\bar{N}_{z}=9$ of cluster members with measured redshifts per cluster, allowing for the elimination of the background contamination. At the same time, only less than half of the clusters in LEB, NED and AND have $N_{z} \geq 3$.

We can use MXS as a control sample to evaluate the quality of the redshift data in LEB and NED. The comparisons LEB - MXS and NED - MXS for 44 and 42 common 
objects, respectively, show large redshift discrepancies for about $10 \%$ of the compared clusters in both cases. We conclude that (1) the compilations LEB and NED contain similar amounts of large redshift errors, and (2) such errors strongly affect only a comparatively small part of the clusters. Therefore, we accept that both compilations are equally suitable for studies of the large $\left(\geq 50 h^{-1} \mathrm{Mpc}\right)$ voids in the spatial distribution of clusters of galaxies.

The combination of the data from LEB, NED, and MXS has been done by assigning different priorities to them. If an object is present in more than one source it obtains the redshift from that source with the higher priority. We give the highest priority to MXS, and lower, but equal priorities to LEB and NED. Because of this equality we merge the data into two parallel compilations: in the first one the objects which are both in LEB and NED have the redshifts from LEB (compilation CL), and in the second one the redshifts from NED (compilation CN). After adding the data from AND, the two versions of the final compilation contain 1868 objects each, 1014 of which are A/ACO clusters $(\sim 45 \%$ of all A/ACO clusters in the $\mathrm{NGH}$ ). The different data sources contribute to compilations CL and CN as follows: MXS - $62 \mathrm{~A} / \mathrm{ACO}$ clusters both in CL and CN, LEB - 1436 objects (812 A/ACO clusters) in CL and 679 (150) in CN, NED - 267 (37) in CL and 1024 (699) in CN, AND - 103 A/ACO clusters both in CL and CN.

The combination of several sources has led to a $25 \%$ increase in comparison with the largest compilation LEB (1 500 clusters and groups). For the A/ACO clusters alone, the increase is about 10\% compared to AND (944 clusters). Consequently, our compilations offer the possibility of a more complete mapping of the large voids in the spatial distribution of concentrations of galaxies in the NGH.

Besides the two compilations CL and CN we have composed also a compilation of galaxies, hereafter referred to as CG, by merging the data from CfA (ZCAT) and MXS. CG contains 22720 galaxies with $b \geq 0^{\circ}$. It is used in Sect. 6 to examine how galaxies populate the voids in the cluster distribution.

In order to define a volume suitable for void investigations we have first studied the variations of the surface and spatial number densities with decreasing galactic latitude and with increasing redshift, respectively, for the A/ACO clusters from the compilations CL and CN.

The surface number density as a function of galactic latitude for $5^{\circ}$ bins in $b$ is shown in Fig. 2 for samples from CL separately for all A/ACO clusters (richness class $R \geq 0$, including the ACO $\mathrm{S}$ clusters) and for the $R \geq 1$ clusters. It is well seen that the effect of galactic obscuration is not strong down to $b=+40^{\circ}$.

The spatial number density as a function of redshift (Fig. 3) is computed for concentric shells of thickness $\Delta z=0.01$. Only objects with $b \geq+30^{\circ}$ have been considered in order to reduce the effect of galactic obscuration. Figure 3 a shows the variation of the number density with

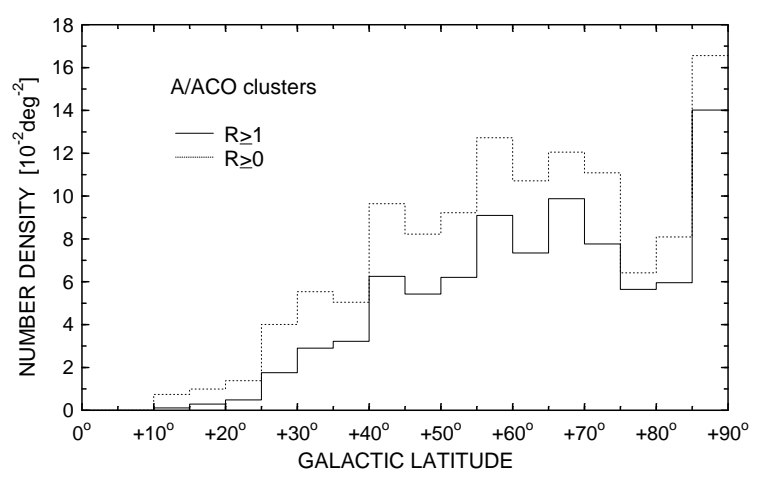

Fig. 2. Surface number density as a function of galactic latitude for A/ACO clusters of richness classes $R \geq 1$ and $R \geq 0$

$z$ for the $R \geq 1$ and $R \geq 0 \mathrm{~A} / \mathrm{ACO}$ clusters from CL. As can be seen, the spatial density of the $R \geq 1$ clusters remains comparatively high up to $z \approx 0.09$. In this range the average density is in good agreement with the estimate by Bahcall \& Cen (1993) $\bar{n}=610^{-6} h^{3} \mathrm{Mpc}^{-3}$ for the mean spatial density of the $R \geq 1 \mathrm{~A} / \mathrm{ACO}$ clusters. In the redshift range $0.09-0.14$ the density of the $R \geq 1$ clusters with measured redshifts drops by more than $50 \%$, remaining, however, comparatively constant in this range. For $z>0.14$ the density is below $25 \%$ of the mean estimate of Bahcall \& Cen.

Figure $3 \mathrm{a}$ shows that the spatial distribution of the $R \geq 0 \mathrm{~A} / \mathrm{ACO}$ clusters is affected by the observational selection even in the redshift range $z<0.09$. This is expected since the $R=0$ clusters represent an incomplete class of objects in Abell's catalogue. While the density of the $R \geq 0$ clusters for $z=0.04-0.09$ agrees well with the estimate by Bahcall \& Cen (1993) $\bar{n}=$ $13.510^{-6} h^{3} \mathrm{Mpc}^{-3}$ for this class of objects, in the nearest volume for $z<0.04$ the density agrees better with the more recent estimate $\bar{n}=2610^{-6} h^{3} \mathrm{Mpc}^{-3}$ of Einasto et al. (1997). For $z>0.09$ the relative decrease of the density is stronger than for the $R \geq 1$ clusters.

We conclude from the examination of Figs. 2 and $3 \mathrm{a}$ that (1) both the $R \geq 1$ and $R \geq 0 \mathrm{~A} / \mathrm{ACO}$ clusters show a weak effect of the galactic obscuration for $b \geq+40^{\circ}$, and (2) the sample of $R \geq 1 \mathrm{~A} / \mathrm{ACO}$ clusters seems to be complete up to $z \sim 0.09$ while the sample of $R \geq 0$ clusters is incomplete even in this closer range.

\subsection{Clusters with estimated redshifts}

One possibility to compensate for the growing incompleteness with distance of the clusters is the usage of estimated distances to the clusters with unmeasured redshifts. A disadvantage of this approach are the large errors $(20-30 \%)$ of the estimated redshifts (Peacock \& West 1992). The unfavourable effect from the large errors may be reduced by keeping small the fraction of clusters with estimated 

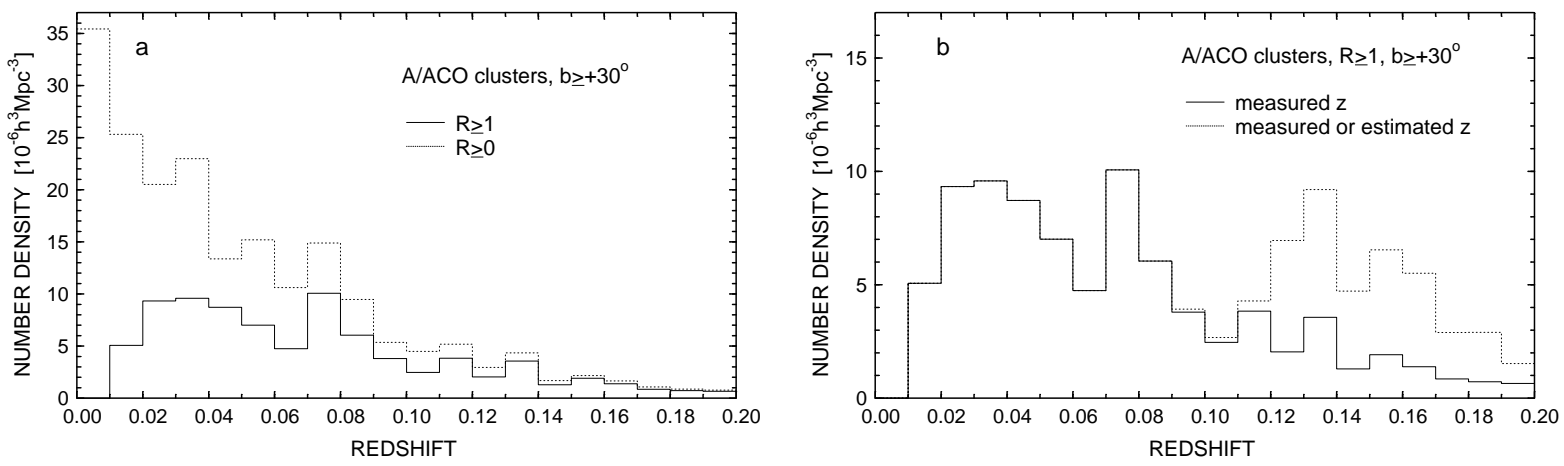

Fig. 3. Spatial number density as a function of redshift for: a) A/ACO clusters of richness classes $R \geq 1$ and $R \geq 0$, b) $R \geq 1$ A/ACO clusters with measured redshifts, compared with that for the $R \geq 1 \mathrm{~A} / \mathrm{ACO}$ clusters with measured or estimated redshifts

redshifts when combining them with clusters with measured redshifts.

To estimate the distances to the $\mathrm{A} / \mathrm{ACO}$ clusters we use the calibration equation (Kalinkov et al. 1985)

$\log z=-4.5372+0.2132 m_{10}$,
$\pm 829 \quad \pm 50$

obtained for a sample of 529 Abell clusters with measured redshifts, where $m_{10}$ is the magnitude of the 10th brightest cluster member from Abell (1958).

We have applied this calibration for 1223 A/ACO clusters in the NGH $\left(b \geq 0^{\circ}\right)$ for which no measured redshifts are available. The clusters with photometrically estimated redshifts have been mixed with the clusters with measured redshifts from compilations CL and CN. It is seen from Fig. $3 \mathrm{~b}$, where the spatial number density of the $R \geq 1$ A/ACO clusters with measured or estimated redshifts is compared with that of the same type of clusters with measured redshifts (from CL), that after the addition of the clusters with estimated $z$ a deep minimum in the number density distribution is formed at $z=0.10-0.11$, followed by a strong enhancement for $z=0.12-0.14$. The latter feature is probably due to systematic effects in Abell's catalogue (Abell 1958). A similar density enhancement in the distance range $300-450 h^{-1} \mathrm{Mpc}$ has been found by Scaramella et al. (1991) for a sample of Abell clusters, about $45 \%$ of which had estimated redshifts, and has been explained as an effect of a systematic overestimate of $m_{10}$ for the distant clusters in Abell's catalogue. However, in spite of the large errors of the estimated distances, the reality of the maximum in this range cannot be definitely ruled out. It is interesting that the density enhancement in the range $0.12-0.14$ coincides well with the third of the peaks in the number of galaxies towards the NGP discovered in the one-dimensional deep survey of Broadhurst et al. (1990).

If the density distribution is cut at $z=0.14$ the corresponding selection function for the $R \geq 1 \mathrm{~A} / \mathrm{ACO}$ clusters with measured or estimated redshifts becomes flat at a density level of $610^{-6} h^{3} \mathrm{Mpc}^{-3}$, in good agreement
Table 1. Cluster samples for $b \geq+20^{\circ}$ and $z \leq 0.16$

\begin{tabular}{clc}
\hline Tracer type & Sample & $\begin{array}{c}\text { Number } \\
\text { of objects }\end{array}$ \\
\hline A/ACO clusters, $R \geq 1$ & AR/L & 415 \\
& ARE/L & 813 \\
& AR/N & 415 \\
& ARE/N & 813 \\
A/ACO clusters, $R \geq 0$ & A/L & 709 \\
& AE/L & 1455 \\
& A/N & 706 \\
& AE/N & 1452 \\
\hline
\end{tabular}

with the Bahcall \& Cen estimate. Therefore, we conclude that this redshift limit is appropriate for the samples containing clusters with measured or estimated redshifts.

Let us note that if the deep minimum at $z=0.10-0.11$ in Fig. $3 \mathrm{~b}$ is real then the sample of $R \geq 1 \mathrm{~A} / \mathrm{ACO}$ clusters with measured redshifts may be complete to $z \approx 0.12$, i.e. nearly $100 h^{-1} \mathrm{Mpc}$ more than the adopted completeness limit $z=0.09$.

\subsection{The cluster samples}

Taking into account the analysis of the completeness of the compilations $\mathrm{CL}$ and $\mathrm{CN}$, we define a volume $V 2$ in the NGH with $b \geq+30^{\circ}$ and $z \leq 0.14$ in which the $R \geq 1 \mathrm{~A} / \mathrm{ACO}$ clusters with measured or estimated redshifts form a complete sample and delimit in it a subvolume $V 1$ with $b \geq+40^{\circ}$ and $z \leq 0.09$ in which the sample of $R \geq 1 \mathrm{~A} / \mathrm{ACO}$ clusters with measured redshifts is complete. To reduce the boundary effects we introduce additionally an enlargement of volume $V 2$ down to $b=+20^{\circ}$ and up to $z=0.16$. While the void search is limited to volume $V 2$ (i.e. void centres must lie in $V 2$ ), the objects outside of volume $V 2$ are used only to constrain the voids which intersect the volume boundaries. 

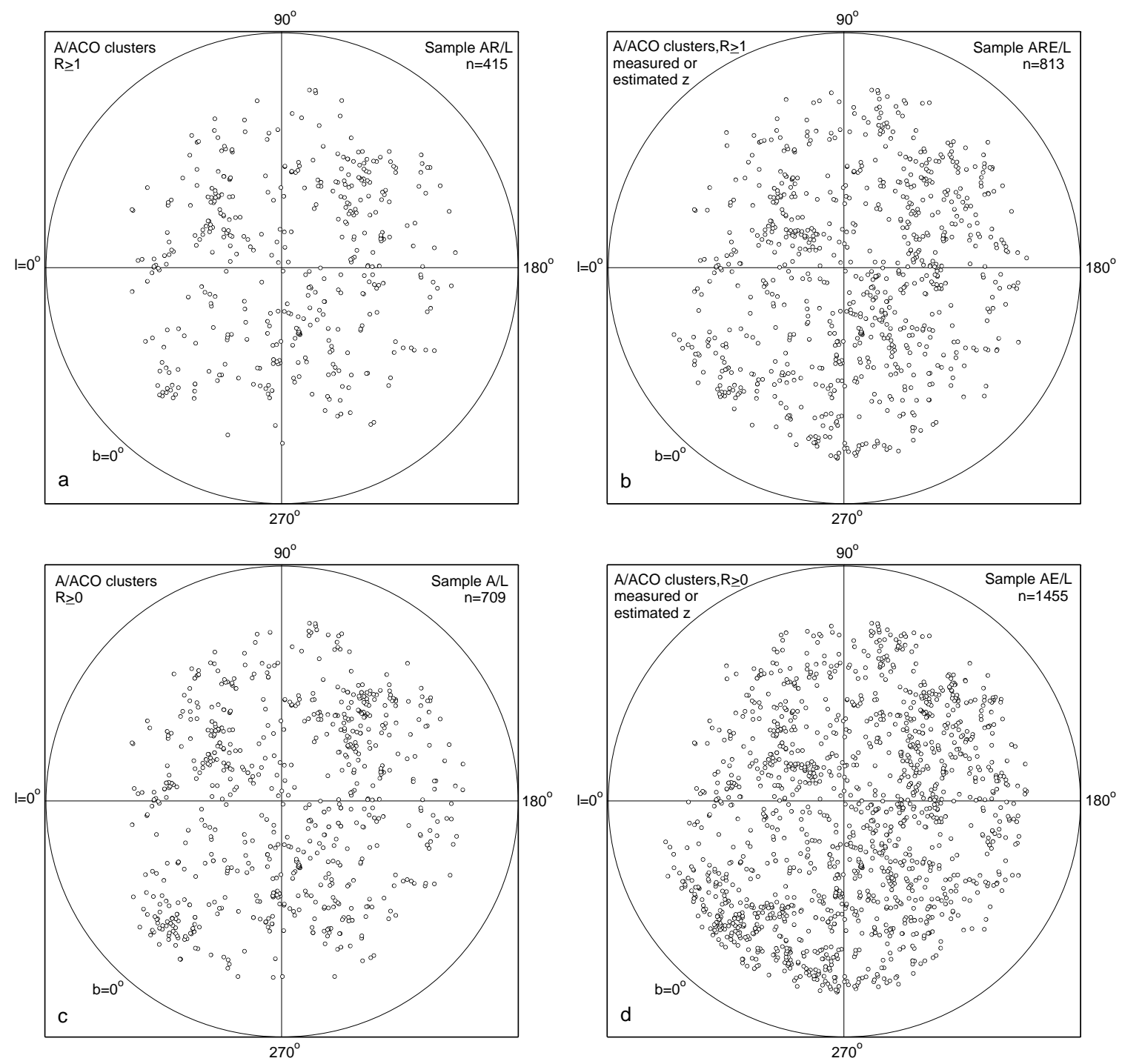

Fig. 4. Surface distributions in galactic coordinates for samples of A/ACO clusters with $b \geq+20^{\circ}$ and $z \leq 0.16$ (see Table 1 ) in Lambert equal-area projection centred on the NGP: a) $R \geq 1$ clusters with measured redshifts, b) $R \geq 1$ clusters with measured or estimated redshifts, $\mathbf{c}$ and $\mathbf{d}$ ) same as a and b) but for the $R \geq 0$ clusters

The samples used in the present study to search for voids are formed by extracting from the compilations CL and $\mathrm{CN}$ all $\mathrm{A} / \mathrm{ACO}$ clusters with $b \geq+20^{\circ}$ and $z \leq 0.16$ separately for (1) the $R \geq 1$ clusters, and (2) all $(R \geq 0)$ $\mathrm{A} / \mathrm{ACO}$ clusters, and for each of these two tracer types separately for the clusters (1) with measured redshifts, and (2) with measured or estimated redshifts. Thus, a total of 8 samples are composed. Table 1 contains the adopted sample designations and the number of objects in each sample. Letter " $\mathrm{L}$ " or "N" in the sample designation means extraction from CL or CN, respectively, and "E" means that the sample contains clusters with estimated redshifts.

For $z \leq 0.14$ the fraction of clusters with estimated redshifts is about $1 / 3$ of the total number $(33 \%$ for the $R \geq 1$ clusters and $36 \%$ for the $R \geq 0$ clusters). However, for $z>0.12$ the number of clusters with estimated redshifts is already larger than that of the measured clusters (see Fig. 3b). There are almost no clusters with only estimated redshifts in the closeby subvolume $V 1$.

Figures $4 \mathrm{a}-\mathrm{d}$ and $5 \mathrm{a}-\mathrm{d}$ show $2-\mathrm{D}$ and 3 -D visualizations, respectively, of the samples from Table 1 (only the samples extracted from CL are shown). The 2-D distributions are Lambert equal-area projections, centred on the NGP, while the $3-\mathrm{D}$ visualizations are perspective graphs.

The 2-D distributions (Fig. 4) show that the NGH is covered satisfactorily by the observations with the exception of the sparsely populated region at low galactic latitudes around $l=0^{\circ}$ affected strongly by the galactic obscuration (Abell 1958; Bahcall \& Soneira 1982b). The inclusion of clusters with estimated redshifts (Figs. 5b and d) increases the completeness but some stratification in 


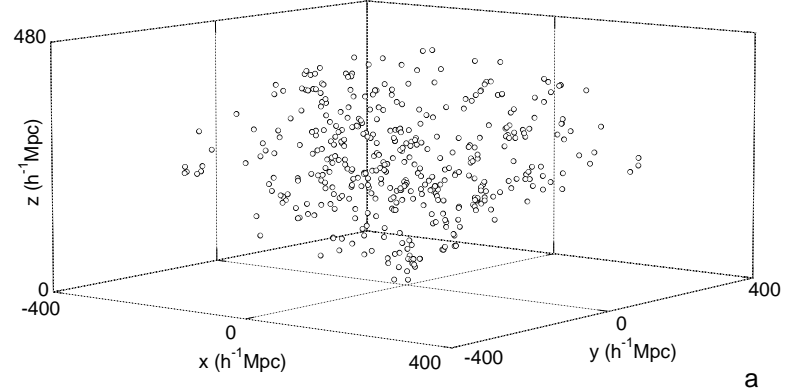

A/ACO clusters, $R \geq 1$, Sample AR/L ( $=415)$

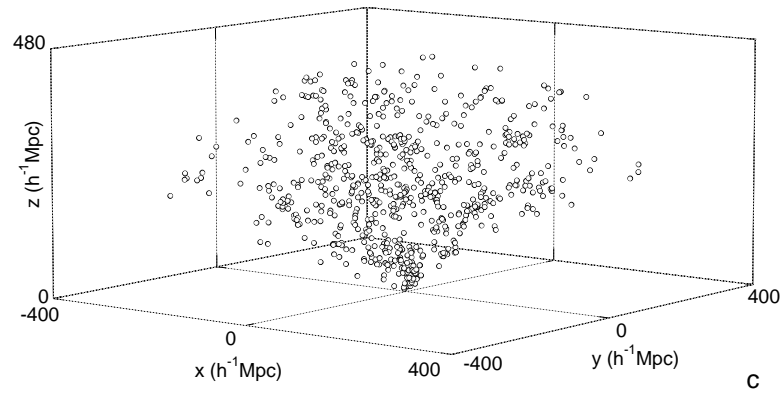

A/ACO clusters, $R \geq 0$, Sample A/L ( $n=709)$

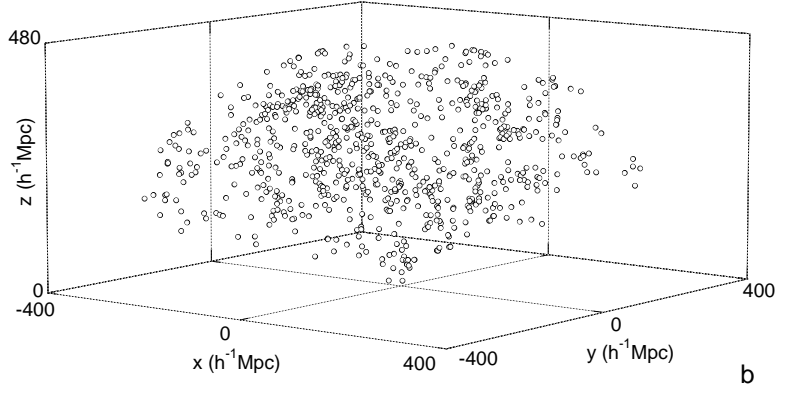

A/ACO clusters, $R \geq 1$, measured or estimated $z$, Sample ARE/L $(n=813)$

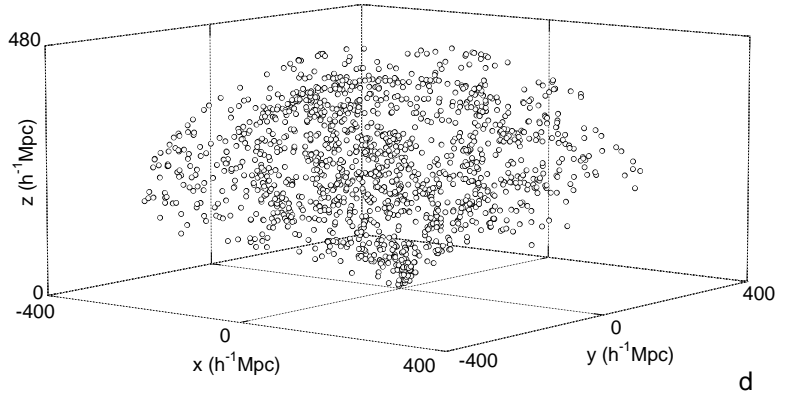

A/ACO clusters, $R \geq 0$, measured or estimated $z$, Sample $A E / L(n=1455)$

Fig. 5. Spatial distributions for samples of A/ACO clusters with $b \geq+20^{\circ}$ and $z \leq 0.16$ (see Table 1) shown as perspective graphs with the coordinate system centred on the Galaxy, axes $x$ and $y$ directed towards $l=0^{\circ}, b=0^{\circ}$, and $l=90^{\circ}, b=0^{\circ}$, respectively, axis $z$ directed towards the NGP, and location of the view point at $b \approx+18^{\circ}, l \approx 320^{\circ}$ : a) $R \geq 1$ clusters with measured redshifts, b) $R \geq 1$ clusters with measured or estimated redshifts, $\mathbf{c}$ and $\mathbf{d}$ ) same as a and $\mathbf{b}$ ) but for the $R \geq 0$ clusters

the spatial distribution appears at larger distances, most probably due to the systematic overestimate of $m_{10}$ in Abell's catalogue.

\section{Automated void search and analysis}

In order to facilitate the quantitative studies of voids we have developed an Automated Void Search and Analysis System (AVSAS). Applications of earlier versions of this system are given in Stavrev (1990a, 1990b, 1990c, 1991, 1998).

AVSAS is a programme package of about 50 modules executing the following functions: (1) preliminary data analysis - homogenization, comparison and combination of data from different sources; (2) local analysis of galaxy and cluster samples - construction and visualization of the nearest-neighbour distance field, search for and parameterization of voids (generation of void catalogues), visualization of the $2-\mathrm{D}$ and $3-\mathrm{D}$ distributions of voids, comparison of void catalogues, identification of the void population and the shell population, construction of void profiles; (3) statistical analysis - statistical comparison of observed samples with random samples, statistical analysis of void catalogues.

\subsection{The void-search algorithm}

The void-search algorithm in AVSAS is based on a definition of the voids as space regions completely devoid of a certain type of objects (clusters, groups, galaxies).

To construct the search algorithm we introduce the concept of a distance field (Stavrev 1990a). Such an approach has been applied also by Frisch et al. (1995), Lindner et al. (1995), and recently by Aikio \& Mähönen (1998).

Let $S$ be a sample of $n$ objects $\mathrm{A}_{i}, i=1, \ldots, n$, with Cartesian coordinates $x_{i}, y_{i}, z_{i}$ in a 3 -D coordinate system as described in Sect. 2.1. We construct in the spatial volume $V_{S}$ of sample $S$ a cubic grid $G$, oriented along the axes $x, y, z$, with grid constant $k\left[h^{-1} \mathrm{Mpc}\right]$ and grid nodes $g_{j}$ with coordinates $x_{j}, y_{j}, z_{j}$, where $j=1, \ldots, m$. For each node the distance to its nearest neighbouring object is computed from

$d_{j}=\min _{i}\left\{\left[\left(x_{i}-x_{j}\right)^{2}+\left(y_{i}-y_{j}\right)^{2}+\left(z_{i}-z_{j}\right)^{2}\right]^{1 / 2}\right\}$, where $i=1, \ldots, n$. The set of distances $d_{j}$ in the nodes $g_{j}$ forms a 3 -D discrete scalar field $d(x, y, z)$, which we shall call nearest-neighbour distance field, or $d$-field for brevity.

The local maxima of the $d$-field indicate regions devoid of objects, while the local minima indicate regions populated by objects. Thus, the task of identification of voids is 
reduced to the task of determination of the local maxima of a 3-D field.

The local maxima (LM) of the $d$-field correspond in real space to the centres of the largest empty spheres embedded in the voids. A better approximation of a void (its position, dimension, shape) can be achieved by a system of crossing (overlapping) empty spheres, i.e. by a group of grid nodes defining a local enhancement of the $d$-field (Stavrev 1991; see also Stavrev 1990c). Similar methods for void search have been developed by El-Ad et al. (1996) and Aikio \& Mähönen (1998). The approximation of a void by more than one sphere is shown schematically in Fig. 6 .

To determine the LM we introduce first a threshold value $d_{\min }$ corresponding to the radius of the smallest voids which are to be identified, i.e. the search for the LM is restricted over the points of the $d$-field with $d \geq d_{\min }$. The neighbourhood of a point of the $d$-field with value $d_{i}$ in which a LM is searched is a sphere with radius $r_{i}$ just equal to $d_{i}$. This means that the search procedure uses a variable neighbourhood which depends on the local properties of the distribution of objects: the neighbourhood is larger in the more sparsely populated regions and smaller in the denser regions. This feature of the procedure makes it suitable for processing spatial distributions of incomplete samples of objects when the number density varies strongly depending on the distance or the galactic latitude.

AVSAS offers two different methods for determining the LM: (1) by direct comparison of the $d$-field values in the neighbourhood of the $d$-field points, and (2) by a criterion $\nu \sigma$ for the standard deviation of the $d$-field values in the neighbourhood of the $d$-field points.

Let $d_{i}^{0} \geq d_{\min }$ be the value of the $d$-field in the running node $g_{i}^{0}$ which has neighbourhood $V_{i}^{0}$ with radius $d_{i}^{0}$, where $i=1, \ldots, m_{1}, m_{1} \leq m\left(m_{1}\right.$ is the number of nodes with $\left.d \geq d_{\text {min }}\right)$. Let $V_{i}^{0}$ contain $N$ nodes with values $d_{j}$, $j=1, \ldots, N$. We define $d_{i}^{0}$ as a local maximum point (i.e. a point in the region of a local maximum of the $d$-field) in $V_{i}^{0}$ if for all $j$

$d_{i}^{0} \geq \frac{d_{j}}{1+p}$,

where $p \geq 0$.

If the parameter $p=0$ the above condition reduces to $d_{i}^{0} \geq d_{j}$ for all $j$. In this case $d_{i}^{0}$ is an absolute maximum in $V_{i}^{0}$ and corresponds to the largest empty sphere embedded in the void.

If $p>0$ the algorithm identifies $d_{i}^{0}$ as a local maximum point not only when it is an absolute maximum in $V_{i}^{0}$ but also when it is smaller by less than $p \times 100 \%$ than the maximum value of the $d$-field in $V_{i}^{0}$. In this case the algorithm describes the LM of the $d$-field by a larger number of points than for $p=0$. Thus, a void is identified (in the next stages of the procedure) as a group of LM points, i.e. the void is approximated by a system of crossing empty spheres.

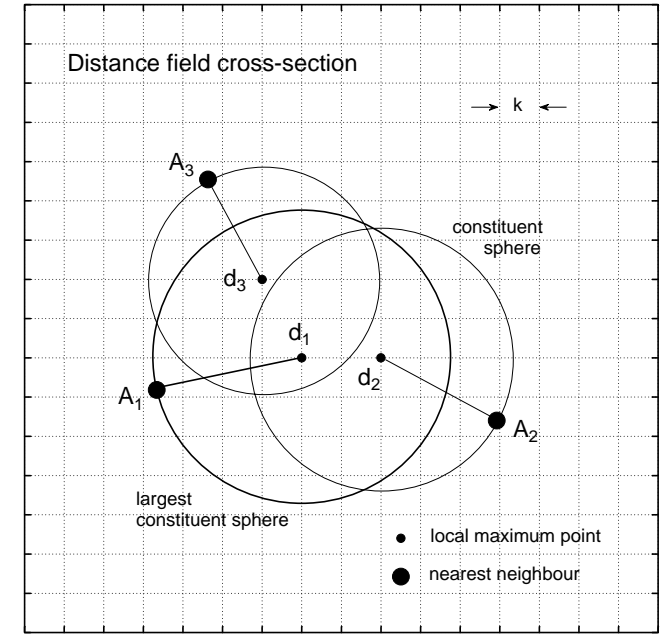

Fig. 6. A schematic representation of the multi-sphere method for the void search. A cross-section of the distance field defined on a grid with constant $k$ is shown with three local maximum points $d_{1}, d_{2}, d_{3}$ and their nearest neighbours $A_{1}, A_{2}, A_{3}$, defining three constituent spheres of a void, one of which is the largest empty sphere

We shall call hereafter the empty spheres constituting a void constituent spheres (CS). For convenience, the method for the determination of the LM points by direct comparison using the parameter $p$ will be called $p$-method.

The second method optionally used in AVSAS for the determination of the LM points consists of the following. First, we calculate the mean of the $d$-field in the neighbourhood $V_{i}^{0}$ of the running node $g_{i}^{0}$

${\overline{d_{i}}}^{0}=\frac{1}{N} \sum_{j=1}^{N} d_{j}$

and the standard deviation

$\sigma_{i}^{0}=\left\{\frac{1}{N-1}\left[\sum_{j=1}^{N} d_{j}^{2}-\frac{1}{N}\left(\sum_{j=1}^{N} d_{j}\right)^{2}\right]\right\}^{1 / 2}$.

Then, $d_{i}^{0}$ is a LM point in $V_{i}^{0}$ if

$d_{i}^{0} \geq \bar{d}_{i}^{0}+\nu \sigma_{i}^{0}$,

where $\nu \geq 0$. Choosing higher or lower values for the parameter $\nu$ we can tune the search algorithm to select more or less statistically significant voids. We shall call this method $\nu$-method.

Applying any of the two described methods on all $d_{i} \geq d_{\text {min }}$, the search procedure produces a set of LM points (or CS). Part of them lie at the boundaries of the examined space volume. These peripheral LM points can optionally be removed from further analysis if it is judged that they are of low significance because of boundary effects or/and sample incompleteness.

In the next stage, the search algorithm groups the LM points on the basis of criteria for their mutual positions. 
Each defined group of LM points constitutes a separate void in the spatial distribution of the sampled objects. The process of grouping of the LM points is complicated by the percolation of the neighbouring voids, especially when samples of rare tracers of the LSS, such as the rich clusters of galaxies, are used. Often the percolation is strengthened by the sample incompleteness.

Because of the uncertainty in delimiting neighbouring voids the search algorithm allows for a certain degree of overlap among them. It offers a choice between three options for grouping the LM points: (1) compact grouping, (2) medium-compact grouping, and (3) loose grouping. According to the first option two LM points affiliate to the same group if the distance between their positions $\Delta r=k$, where $k$ is the grid constant. This option leads to compact groups of a comparatively small number of LM points, and consequently a large number of voids with a high degree of percolation of neighbouring voids. The criterion for the medium-compact grouping is $\Delta r \leq \sqrt{3} k$ (i.e. a distance equal to the diagonal of the elementary grid cube). This option produces voids with more complicated configurations of CS and less overlap with neighbouring voids than the first option. The criterion for the third option - loose grouping -, unlike the first two options, does not depend on $k$ : two LM points with values $d_{1}$ and $d_{2}$ are grouped together if $\Delta r<\max \left(d_{1}, d_{2}\right)$. This criterion assures that the center of at least one of the two CS lies within the other CS. The voids produced by loose grouping of LM points may have complicated (irregular) shapes and a large number of CS, i.e. large sizes. However, the overlap of neighbouring voids is less (relative to void size) compared with the first two options.

The voids identified with option 1 can be considered as substructures of the voids identified with options 2 and 3 , and vice versa - the voids identified with option 3 can be considered as unifications of the voids identified with options 1 and 2 .

The choice of the grouping option from 1 to 3 acts parallel to the choice of a higher value of the parameter $p$ and a lower value of the parameter $\nu$, i.e. towards larger voids with more complicated shapes.

As is seen from the above the void-search algorithm needs choices of suitable values for the following free parameters: $k, d_{\min }, p / \nu$, and the grouping option. The question arises: given the other values, what values of $p$ and $\nu$, respectively, are most suitable for the void search? To answer this question a number of tests of the $p$ method and the $\nu$-method have been carried out over two test samples $(\mathrm{AR} / \mathrm{L}$ and $\mathrm{AR} / \mathrm{N}$, limited to volume $V 2$, see Sect. 2.3) using two values for $k$ (10 and $20 h^{-1} \mathrm{Mpc}$ ), two values for the minimum void diameter $D_{\min }=2 d_{\text {min }}$ (50 and $80 h^{-1} \mathrm{Mpc}$ ), and two grouping options (mediumcompact and loose grouping). Then, for a certain combination of $k, D_{\min }$, and the grouping option we check how the number of identified voids and CS changes as a function of $p$ or $\nu$.
The values of $D_{\min }$ have been chosen to be roughly equal to or larger than the mean separation of clusters which is $\sim 53 h^{-1} \mathrm{Mpc}$ and $\sim 42 h^{-1} \mathrm{Mpc}$ for the $R \geq 1$ and $R \geq 0$ Abell clusters, respectively (Batuski et al. 1991). With these $D_{\min }$ the values of $k$ are chosen to be sufficiently small, so that the LM corresponding to the smallest identified voids can be determined from statistics over at least several dozens of $d$-field points. The only combination for which this requirement is not fulfilled is the one with $D_{\min }=50 h^{-1} \mathrm{Mpc}, k=20 h^{-1} \mathrm{Mpc}$.

The choice of a sufficiently small value of $k$ is also of importance for the accuracy of the determined void parameters. From elementary considerations follows that the error of the positions of the CS centres due to the discreteness of the $d$-field can be estimated as $\frac{\sqrt{3}}{4} k$. For the two chosen values of $k\left(20\right.$ and $\left.10 h^{-1} \mathrm{Mpc}\right)$ the error is 8.66 and $4.33 h^{-1} \mathrm{Mpc}$, respectively. In the latter case the error is comparable to the diameter of Abell clusters $\left(3 h^{-1} \mathrm{Mpc}\right)$, and it is less than $10 \%$ of the diameter of the smallest identified voids $\left(50 h^{-1} \mathrm{Mpc}\right)$. Let us also note that the background contamination, the large peculiar velocities of the cluster members, and the use of estimated redshifts may lead to positional errors larger than both chosen values for $k$.

The results from the tests are shown in Fig. 7. Meaningful values of $p$ and $\nu$ lie in the ranges $p \lesssim 0.20$ and $\nu \gtrsim 1.0$. Outside these ranges the search procedure produces a too large number of LM points and this causes difficulties in delimiting neighbouring voids because of increased overlap among them. For the case of the medium-compact grouping (Fig. 7, left panel) the number of voids increases continuously with increasing $p$ and decreasing $\nu$, while for the loose grouping (Fig. 7, right panel) the curves show well defined maxima in the number of voids. For values of $p \approx 0$ and $\nu \approx 2.6-2.7$ the search algorithm identifies only the most significant voids - deep, spherical and well isolated regions, approximated by single empty spheres. The increase of $p$, respectively the decrease of $\nu$, relaxes the criterion for the selection of LM points, hence the number of voids grows. At the same time, voids enlarge and start to overlap more due to the increased number of CS. This causes the merging of neighbouring voids and consequently a decrease of the number of voids. In the case of the loose grouping of the LM points, for certain values of the parameters $p$ and $\nu$ the process of merging overtakes the increase of the number of LM points, hence the number of voids decreases. If $p$ is left to grow very large or $\nu$ to become very small the number of voids tends to 1: a single, continuous, network structure is formed. For the medium-compact option the process of merging is not strong enough to stop the growth of the number of voids with the growth of $p$, respectively with the decrease of $\nu$. (This is true for the case of compact grouping of the LM points, too.) 

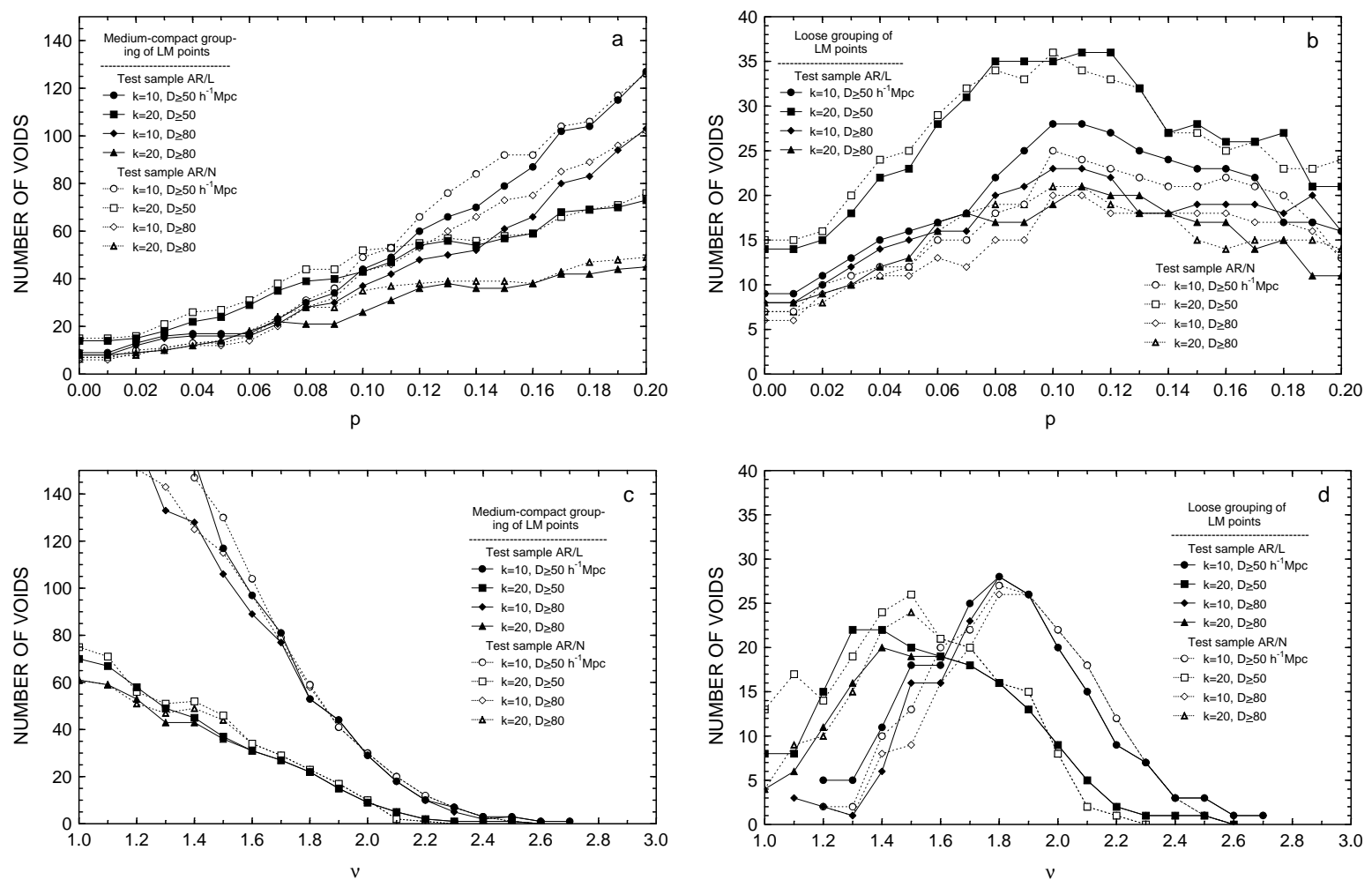

Fig. 7. Number of voids as functions of the parameters $p$ a and b) and $\nu \mathbf{c}$ and $\mathbf{d}$ ) for medium-compact (left panel) and loose (right panel) grouping of the local maximum points, for two test samples (AR/L and AR/N) and four combinations of the grid constant $k$ and the minimum void dimension $D_{\text {min }}$

The test curves for the $p$-method, loose grouping (Fig. 7b) show maxima approximately for the same value $p_{\mathrm{m}}=0.10-0.12$. The $\nu$-method, however, shows two distinct maxima depending on the value of $k: \nu_{\mathrm{m}}=1.8$ for $k=10 h^{-1} \mathrm{Mpc}$ and $\nu_{\mathrm{m}}=1.4-1.5$ for $k=20 h^{-1}$ Mpc. This stronger dependence of the $\nu$-method on $k$ in comparison with the $p$-method is well outlined in Fig. 7c. On the other hand, it is seen from Figs. $7 \mathrm{a}-\mathrm{d}$ that the $\nu$-method is less dependent on $D_{\min }$ than the $p$-method.

Figure $7 \mathrm{~b}$ shows that for the $p$-method the combination $k=20 h^{-1} \mathrm{Mpc}, D_{\min }=50 h^{-1} \mathrm{Mpc}$ leads to a substantially higher number of voids than all other combinations. This case differs from the other three combinations by its very low ratio $D_{\min } / k=2.5$. As a result part of the LM points are defined in neighbourhoods $V_{i}^{0}$ containing a very small number of grid points. This probably has the effect of relaxing the criterion for the selection of voids, hence increasing the number of voids. The $\nu$-method is less sensitive to this effect. We conclude that values of $D_{\min } / k$ lower than $4-5$ should be avoided, especially when the $p$ method is used.

If we exclude this peculiar case and compare the number of voids for all other cases at $p_{\mathrm{m}}$ (Fig. $7 \mathrm{~b}$ ) with the number of voids at $\nu_{\mathrm{m}}$ (Fig. $7 \mathrm{~d}$ ) we see that for both methods the number of voids is in the same range of about
20 - 30 depending slightly on the combination $\left(k, D_{\min }\right)$, i.e. the agreement between the two methods is good.

In order to check how $p_{\mathrm{m}}$ and $\nu_{\mathrm{m}}$ depend on the number density we have used test samples with growing numbers of objects in the same volume. These tests show that $p_{\mathrm{m}}$ decreases slightly with increasing number density, while $\nu_{\mathrm{m}}$ is almost independent of it. The reason for this difference between the two methods is that the $\nu$ method is more efficient than the $p$-method at rejecting the smaller voids (near $D_{\min }$ ) in the denser regions as insignificant fluctuations of the $d$-field. For the $p$-method the increase of the sample density leads to an increase of the number and the overlap of the CS, hence the number of voids decreases. This effect is compensated by a smaller value of $p_{\mathrm{m}}$ which tightens the criterion for selection of LM points.

The tests of the void-search procedure suggest that it can be optimized by choosing values of $p=p_{\mathrm{m}}$ and $\nu=\nu_{\mathrm{m}}$. That leads to the detection of the maximum number of voids at a low degree of overlapping of the neighbouring voids in the case of the loose grouping of the LM points. These values of $p$ and $\nu$ can be applied as well in the other two options - compact and mediumcompact grouping, in spite of the fact that the number of detected voids is not maximized, but for the sake of 
keeping a low degree of overlapping of the neighbouring voids, hence better delimited voids.

\subsection{Determination of the void parameters}

After voids are identified, AVSAS analyses their properties and defines the following parameters:

(1) Cartesian, equatorial and galactic coordinates of the CS centres and of the void centre. The latter is defined as the centre of the largest CS of the void, and alternatively, as the centroid of all CS of the void. The centroid is determined as the centre of gravity of the system of CS. If a void is composed of $n_{\mathrm{cs}}>1 \mathrm{CS}$, each one with volume $V_{i}, i=1, \ldots, n_{\mathrm{cs}}$, and weight

$w_{i}=V_{i} / \sum_{j=1}^{n_{\mathrm{cs}}} V_{j}$,

then the Cartesian coordinates of the centroid of CS are computed from

$x_{\mathrm{c}}=\sum_{i=1}^{n_{\mathrm{cs}}} w_{i} x_{i}, \quad y_{\mathrm{c}}=\sum_{i=1}^{n_{\mathrm{cs}}} w_{i} y_{i}, \quad z_{\mathrm{c}}=\sum_{i=1}^{n_{\mathrm{cs}}} w_{i} z_{i}$,

where $x_{i}, y_{i}, z_{i}$ are the Cartesian coordinates of the $i$-th CS. For convenience, the centroids are slightly shifted in order to coincide with the nearest grid node.

The Cartesian coordinates $x_{\mathrm{L}}, y_{\mathrm{L}}$ in a Lambert equalarea projection of the CS centres and of the centroid are computed, too, as described in Sect. 2.1.

(2) Distances are computed to the centres of all CS, as well as to the CS centroid from the Cartesian coordinates: $r_{i}=\sqrt{x_{i}^{2}+y_{i}^{2}+z_{i}^{2}} \quad\left[h^{-1} \mathrm{Mpc}\right]$,

and

$r_{\mathrm{c}}=\sqrt{x_{\mathrm{c}}^{2}+y_{\mathrm{c}}^{2}+z_{\mathrm{c}}^{2}} \quad\left[h^{-1} \mathrm{Mpc}\right]$.

(3) Dimensions:

(a) Diameters of the CS are computed directly from the values of the $d$-field as $D_{i}=2 d_{i} \quad\left[h^{-1} \mathrm{Mpc}\right]$.

(b) Void dimensions along $x, y, z$ axes are determined from the projected distances between the centres of those two CS of the void, which are the farthest apart along each axis, plus their radii. Thus, for the $x$ axis

$D_{x}=\Delta_{x}+d_{\mathrm{A}}+d_{\mathrm{B}}\left[h^{-1} \mathrm{Mpc}\right]$.

(c) The maximum void dimension is determined from the distance between the centres of the two most widely separated CS of the void plus the radii of these two CS:

$D_{\max }=\Delta_{\max }+d_{\mathrm{A}}+d_{\mathrm{B}}\left[h^{-1} \mathrm{Mpc}\right]$.

(d) The equivalent void diameter is the diameter of the sphere whose volume is equal to the total volume $V_{\mathrm{T}}$ of the void (see below):

$D_{\mathrm{e}}=\left(\frac{6}{\pi} V_{\mathrm{T}}\right)^{1 / 3} \quad\left[h^{-1} \mathrm{Mpc}\right]$.
(4) Sphericity s. This parameter is introduced for a characterization of the void shape. It is defined as $s=D / D_{\max }$,

where $D$ is the diameter of the largest CS of the void.

(5) Volume:

(a) The volume of each CS is computed from $V_{i}=\frac{4}{3} \pi d_{i}^{3} \quad\left[h^{-3} \mathrm{Mpc}^{3}\right]$

(b) The total void volume $V_{\mathrm{T}}$ is computed numerically because of the complicated void shape (especially when the void is composed of a large number of CS), by counting the number of elementary grid cubes $n_{\mathrm{g}}$ in the void (strictly speaking, the cubes whose centres are inside the void). Then

$V_{\mathrm{T}}=n_{\mathrm{g}} k^{3} \quad\left[h^{-3} \mathrm{Mpc}^{3}\right]$,

where $k$ is the grid constant.

(6) The objects surrounding the void are defined as the nearest neighbours to the centres of the CS of the void. (See Sect. 6 for a more complete identification of the objects surrounding a void.)

(7) The neighbouring voids of the void are determined from a condition for overlapping of at least one CS of the void with at least one CS of the neighbouring void. If $d_{1}$ and $d_{2}$ are the radii of two CS belonging to two different voids, they overlap if $\Delta r<d_{1}+d_{2}$, where $\Delta r$ is the distance between the centres of the two CS. (Note the difference between this criterion and the criterion for loose grouping of the LM points in Sect. 3.1.)

\section{Generation of void catalogues}

We have applied AVSAS over the 8 observational samples described in Sect. 2 (see Table 1) which occupy the same volume. The generated void catalogues contain the voids whose CS have centres lying in volume $V 2$.

All catalogues are generated with the same grid constant $k=10 h^{-1} \mathrm{Mpc}$ (see discussion in Sect. 3.1), producing 77603 grid nodes in volume $V 2$, on which the $d$-field is calculated. The semi-cube containing the conical volume $V 2$ has dimensions $85 k \times 85 k \times 43 k$ along the $x, y, z$ axes, respectively.

The void selection criterion with which a void catalogue is generated is a combination of five conditions for (1) the minimum void diameter $D_{\min }$, (2) the search method $p$ or $\nu,(3)$ the value of the search parameter $p$ or $\nu$, (4) the option for the grouping of the LM points (compact, medium-compact, loose), and (5) the treatment of the peripheral LM points (acceptance or rejection).

Since we are interested in large voids we have chosen $D_{\min }=50 h^{-1} \mathrm{Mpc}$ (see discussion in Sect. 3.1). This dimension is comparable with the characteristic size of the superclusters of galaxies.

We have applied for each sample both search methods, setting $p=p_{\mathrm{m}}$ and $\nu=\nu_{\mathrm{m}}$ to optimize the search. 
Table 2. Void catalogues: number of local maximum points and voids in volumes $V 1$ and $V 2$

\begin{tabular}{|c|c|c|c|c|c|c|c|c|c|c|c|}
\hline \multirow[b]{4}{*}{ No. } & \multirow[b]{4}{*}{ Sample } & \multirow[b]{4}{*}{ Method } & \multirow[b]{4}{*}{$p_{\mathrm{m}} / v_{\mathrm{m}}$} & \multicolumn{2}{|c|}{ LM POINTS } & \multicolumn{6}{|c|}{ VOIDS } \\
\hline & & & & \multirow[b]{3}{*}{$V 1$} & \multirow[b]{3}{*}{$V 2$} & \multicolumn{6}{|c|}{ Grouping of LM points: } \\
\hline & & & & & & \multicolumn{2}{|c|}{ compact } & \multicolumn{2}{|c|}{ medium-compact } & \multicolumn{2}{|c|}{ loose } \\
\hline & & & & & & $V 1$ & $\sqrt{ } 2$ & $V 1$ & $V 2$ & $V 1$ & $V 2$ \\
\hline 1 & AR/L & $p$ & 0.10 & 114 & 402 & 39 & 119 & 20 & 60 & 13 & 36 \\
\hline 2 & ARE/L & $p$ & 0.10 & 115 & 480 & 40 & 160 & 21 & 90 & 12 & 44 \\
\hline 3 & $\mathrm{AR} / \mathrm{L}$ & $v$ & 1.8 & 89 & 450 & 30 & 133 & 17 & 69 & 9 & 26 \\
\hline 4 & $\mathrm{ARE} / \mathrm{L}$ & $v$ & 1.8 & 90 & 511 & 31 & 163 & 18 & 89 & 9 & 36 \\
\hline 5 & $\mathrm{AR} / \mathrm{N}$ & $p$ & 0.10 & 92 & 351 & 38 & 118 & 22 & 65 & 12 & 34 \\
\hline 6 & ARE/N & $p$ & 0.10 & 93 & 454 & 38 & 155 & 22 & 87 & 10 & 42 \\
\hline 7 & $\mathrm{AR} / \mathrm{N}$ & $v$ & 1.8 & 78 & 436 & 29 & 121 & 17 & 72 & 7 & 28 \\
\hline 8 & $\mathrm{ARE} / \mathrm{N}$ & $v$ & 1.8 & 83 & 514 & 31 & 155 & 17 & 88 & 7 & 34 \\
\hline 9 & $\mathrm{~A} / \mathrm{L}$ & $p$ & 0.10 & 128 & 435 & 57 & 162 & 35 & 95 & 20 & 54 \\
\hline 10 & $\mathrm{AE} / \mathrm{L}$ & $p$ & 0.09 & 125 & 512 & 56 & 219 & 35 & 130 & 21 & 70 \\
\hline 11 & $\mathrm{~A} / \mathrm{L}$ & $v$ & 1.8 & 100 & 416 & 46 & 158 & 31 & 91 & 16 & 46 \\
\hline 12 & $\mathrm{AE} / \mathrm{L}$ & $v$ & 1.8 & 108 & 497 & 49 & 202 & 33 & 129 & 16 & 63 \\
\hline 13 & $\mathrm{~A} / \mathrm{N}$ & $p$ & 0.10 & 120 & 442 & 52 & 160 & 34 & 98 & 16 & 42 \\
\hline 14 & $\mathrm{AE} / \mathrm{N}$ & $p$ & 0.09 & 122 & 529 & 57 & 230 & 35 & 128 & 17 & 63 \\
\hline 15 & $\mathrm{~A} / \mathrm{N}$ & $v$ & 1.8 & 106 & 439 & 48 & 159 & 29 & 92 & 14 & 45 \\
\hline 16 & $\mathrm{AE} / \mathrm{N}$ & $v$ & 1.8 & 114 & 502 & 51 & 211 & 30 & 121 & 13 & 60 \\
\hline
\end{tabular}

The values of $p_{\mathrm{m}}$ or $\nu_{\mathrm{m}}$ for each generated catalogue are given in Table 2.

All three options for the grouping of the LM points have been used for each sample and each method to generate void catalogues. Thus, 6 catalogues -3 for the $p$-method and 3 for the $\nu$-method - have been produced for each sample. However, for further void analysis in this paper we have chosen only the voids generated by the medium-compact grouping of the LM points assuming that the compact grouping is more suitable for the study of void substructures, while the loose grouping is suitable for the study of void superstructures (see Sect. 3.1).

All void catalogues have been generated rejecting the peripheral LM points.

The total number of generated void catalogues is 48 . The numbers of voids and LM points in each catalogue are given in Table 2. Hereafter, we shall designate the void catalogues for the medium-compact option by the corresponding sample designation (Table 2, Col. 2) supplemented by the suffix $p$ or $\nu$ for the method applied (Table 2, Col. 3), or we shall simply use the number given in the first column of Table 2 .

Two of the void catalogues listed in Table 2 corresponding to samples $\mathrm{AR} / \mathrm{L}$ and $\mathrm{A} / \mathrm{L}$ are presented in Tables 3 and 4 . They are generated with the $p$-method and the medium-compact grouping of the LM points. Because of the large number of void parameters and void CS the catalogues are given in a concise form, with one or two lines of data for each void: the first line contains (after Col. 2) the parameters of the largest CS of the void, and the second line contains the parameters of the whole void. Only one line appears for voids consisting only of one CS. If the void centre coincides with the centre of the largest $\mathrm{CS}$, the positional parameters are given only in the first line. Voids are ordered by increasing right ascension of the centre of the largest CS.

Tables 3 and 4 have the following contents: Col. (1) void serial number; Col. (2) - number of CS of the void; Cols. (3) and (4) - equatorial coordinates $\alpha, \delta$ for B1950.0 of the centre of the largest CS of the void (line 1), and $\alpha_{\mathrm{c}}, \delta_{\mathrm{c}}$ of the centroid of all CS (line 2); Cols. (5) and (6) - galactic coordinates $l, b$ of the centre of the largest CS (line 1), and $l_{\mathrm{c}}, b_{\mathrm{c}}$ of the centroid of all CS (line 2); Cols. (7)-(9) - Cartesian coordinates $x, y, z$ of the centre of the largest CS (line 1), and $x_{\mathrm{c}}, y_{\mathrm{c}}, z_{\mathrm{c}}$ of the centroid of all CS (line 2); Col. (10) - distance $r$ to the centre of the largest CS (line 1), and $r_{\mathrm{c}}$ to the centroid of all CS (line 2); Col. (11) - position of the centre of the largest CS (line 1) and of the centroid of all CS (line 2) with respect to volumes $V 1$ and $V 2$ : "1" - in volume $V 1$, "2" in volume $V 2$, outside volume $V 1$; Col. (12) - diameter $D$ of the largest CS (line 1), and equivalent void diameter $D_{\text {e }}$ (line 2); Cols. (13)-(15) - void dimensions along axes $x, y, z$; Col. (16) - largest void dimension $D_{\max }$; Col. (17) - volume $V$ of the largest CS (line 1), and total volume of the void $V_{\mathrm{T}}$ (line 2); Col. (18) - sphericity $s$.

The void catalogues listed in Table 2, two of which are given in Tables 3 and 4 , represent the currently most complete mapping of the large voids in the distribution of galaxy clusters in the NGH to a limiting distance of $420 h^{-1}$ Mpc. Compared to similar wide-angle studies (Batuski \& Burns 1985; Tully 1986; Einasto et al. 1994) they contain larger numbers of voids to larger distances. They can be used as identification lists of the voids in the NGH, in studies of individual voids, as well as for statistical investigations of the void properties.

The spatial and surface distributions of the voids in Tables 3 and 4 are presented in Figs. $8-10$ by different types of visualizations allowing for a visual examination and comparison of the void catalogues. The spatial 
Table 3. Voids of $R \geq 1 \mathrm{~A} / \mathrm{ACO}$ clusters (sample AR/L, $p$-method)

\begin{tabular}{|c|c|c|c|c|c|c|c|c|c|c|c|c|c|c|c|c|c|}
\hline No. & $\mathrm{n}_{\mathrm{cs}}$ & $\begin{array}{r}\alpha \\
\alpha_{c} \\
{[h]}\end{array}$ & $\begin{array}{c}\delta \\
\delta_{c} \\
{\left[^{\circ}\right]} \\
\end{array}$ & $\begin{array}{l}1 \\
1 \mathrm{c} \\
{\left[{ }^{\circ}\right]} \\
\end{array}$ & $\begin{array}{c}b \\
b_{c} \\
{\left[{ }^{\circ}\right]}\end{array}$ & $\begin{array}{l}\boldsymbol{x} \\
\boldsymbol{x}_{\mathrm{c}} \\
\\
\end{array}$ & $\begin{array}{c}\bar{Y} \\
y_{\mathrm{c}} \\
\left.h^{-1} \mathrm{Mpc}\right] \\
\end{array}$ & $\begin{array}{l}z \\
z_{\mathrm{c}} \\
\end{array}$ & $\begin{array}{c}r \\
r_{c} \\
{\left[h^{-1} \mathrm{Mpc}\right]}\end{array}$ & $\begin{array}{l}\mathrm{SV} \\
S V_{c}\end{array}$ & $\begin{array}{c}D \\
D_{e} \\
{\left[h^{-1} \mathrm{Mpc}\right]}\end{array}$ & $\begin{array}{l}D_{x} \\
\quad[h\end{array}$ & $\begin{array}{c}D_{y} \\
\left.{ }^{-1} \mathrm{Mpc}\right]\end{array}$ & $D_{z}$ & $D_{\max }$ & $\begin{array}{c}V \\
V_{T} \\
{\left[10^{6} h^{-3} \mathrm{Mpc}^{3}\right]}\end{array}$ & $\begin{array}{l}s \\
s\end{array}$ \\
\hline 1 & 7 & $\begin{array}{l}8.4 \\
8.5\end{array}$ & $\begin{array}{l}30 \\
29\end{array}$ & $\begin{array}{l}193 \\
195\end{array}$ & 33 & -300 & -70 & 200 & 367 & 2 & 204 & & & & & $\begin{array}{r}4.445 \\
5.593\end{array}$ & \\
\hline 2 & 1 & 8.9 & 35 & $\begin{array}{l}195 \\
188\end{array}$ & $\begin{array}{l}34 \\
40\end{array}$ & $\begin{array}{l}.00 \\
70\end{array}$ & $\begin{array}{l}-80 \\
-10\end{array}$ & $\begin{array}{r}210 \\
60\end{array}$ & $\begin{array}{r}375 \\
93\end{array}$ & $\begin{array}{l}2 \\
1\end{array}$ & $\begin{array}{r}216 \\
96\end{array}$ & 218 & 212 & 219 & 223 & $\begin{array}{l}5.293 \\
0.463\end{array}$ & $\begin{array}{l}0.91 \\
1.00\end{array}$ \\
\hline 3 & 31 & $\begin{array}{l}8.9 \\
8.9\end{array}$ & $\begin{array}{l}55 \\
56\end{array}$ & $\begin{array}{l}162 \\
161\end{array}$ & $\begin{array}{l}40 \\
40\end{array}$ & $\begin{array}{l}-180 \\
-170\end{array}$ & $\begin{array}{l}60 \\
60\end{array}$ & $\begin{array}{l}160 \\
150\end{array}$ & 248 & 1 & 164 & & & & 246 & $\begin{array}{l}2.310 \\
3720\end{array}$ & 0.6 \\
\hline 4 & 2 & 9.4 & 13 & 219 & $\begin{array}{l}40 \\
41\end{array}$ & $\begin{array}{l}-170 \\
-100\end{array}$ & $\begin{array}{r}60 \\
-80\end{array}$ & $\begin{array}{l}150 \\
110\end{array}$ & $\begin{array}{l}235 \\
169\end{array}$ & $\begin{array}{l}1 \\
1\end{array}$ & $\begin{array}{l}203 \\
106\end{array}$ & 213 & 190 & 231 & 246 & $\begin{array}{l}4.372 \\
0.624\end{array}$ & 0.67 \\
\hline 5 & 7 & 9.6 & -5 & 241 & 33 & -150 & -270 & & 368 & 2 & $\begin{array}{l}111 \\
182\end{array}$ & 106 & 114 & 114 & 118 & $\begin{array}{l}0.724 \\
3.157\end{array}$ & 0.90 \\
\hline 6 & 4 & $\begin{array}{l}9.7 \\
9.6\end{array}$ & $\begin{array}{l}-5 \\
34\end{array}$ & 243 & 35 & -140 & -270 & 210 & 370 & 2 & 196 & 202 & 192 & 197 & 207 & $\begin{array}{l}3.945 \\
0.624\end{array}$ & 0.88 \\
\hline 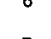 & 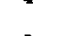 & 9.6 & 34 & 191 & 48 & -160 & -30 & 180 & 243 & & $\begin{array}{l}106 \\
114\end{array}$ & 113 & 106 & 122 & 124 & $\begin{array}{l}0.624 \\
0.778\end{array}$ & 0.85 \\
\hline 7 & 9 & $\begin{array}{l}9.7 \\
9.6\end{array}$ & $\begin{array}{l}25 \\
25\end{array}$ & $\begin{array}{l}204 \\
204\end{array}$ & $\begin{array}{l}49 \\
47\end{array}$ & $\begin{array}{l}-110 \\
-110\end{array}$ & $\begin{array}{l}-50 \\
-50\end{array}$ & $\begin{array}{l}140 \\
130\end{array}$ & $\begin{array}{l}185 \\
177\end{array}$ & $\frac{1}{1}$ & $\begin{array}{l}116 \\
132\end{array}$ & 126 & 132 & 139 & 140 & $\begin{array}{l}0.817 \\
1.206\end{array}$ & 0.83 \\
\hline 8 & 2 & $\begin{array}{l}9.8 \\
9.8\end{array}$ & $\begin{array}{l}15 \\
14\end{array}$ & $\begin{array}{r}220 \\
220\end{array}$ & $\begin{array}{l}47 \\
47\end{array}$ & $\begin{array}{l}-120 \\
-130\end{array}$ & $\begin{array}{l}-100 \\
-110\end{array}$ & $\begin{array}{l}170 \\
180\end{array}$ & 231 & 1 & $\begin{array}{l}82 \\
87\end{array}$ & 92 & 92 & 92 & 99 & $\begin{array}{l}0.289 \\
0.342\end{array}$ & 0.83 \\
\hline 9 & 5 & $\begin{array}{l}9.8 \\
9.7\end{array}$ & $\begin{array}{l}14 \\
42\end{array}$ & $\begin{array}{l}220 \\
178\end{array}$ & $\begin{array}{l}41 \\
50\end{array}$ & $\begin{array}{l}-130 \\
-260\end{array}$ & $\begin{array}{r}-110 \\
10\end{array}$ & 1 & 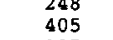 & $\frac{1}{2}$ & $\begin{array}{l}87 \\
134\end{array}$ & 92 & 92 & 32 & 35 & 1.260 & 0.03 \\
\hline 10 & 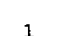 & $\begin{array}{r}9.6 \\
10.2\end{array}$ & 44 & $\begin{array}{l}176 \\
232\end{array}$ & $\begin{array}{l}49 \\
49\end{array}$ & $\begin{array}{l}-260 \\
-100\end{array}$ & $\begin{array}{r}20 \\
-130\end{array}$ & 300 & 397 & 2 & 145 & 146 & 149 & 141 & 155 & 1.589 & 0.86 \\
\hline 11 & 1 & $\begin{array}{l}10.2 \\
10.4\end{array}$ & $\begin{array}{r}8 \\
-18\end{array}$ & $\begin{array}{l}232 \\
262\end{array}$ & $\begin{array}{l}49 \\
33\end{array}$ & $\begin{array}{r}-100 \\
-40\end{array}$ & $\begin{array}{l}-130 \\
-290\end{array}$ & $\begin{array}{l}190 \\
190\end{array}$ & $\begin{array}{l}251 \\
349\end{array}$ & $\begin{array}{l}1 \\
2\end{array}$ & $\begin{array}{r}74 \\
178\end{array}$ & & & & & $\begin{array}{l}0.212 \\
2.953\end{array}$ & $\begin{array}{l}1.00 \\
1.00\end{array}$ \\
\hline 12 & 2 & 10.4 & 15 & 225 & 56 & -160 & -160 & 330 & 400 & 2 & $\begin{array}{l}184 \\
190\end{array}$ & 192 & $10 ?$ & 107 & 109 & $\begin{array}{r}3.262 \\
3.606\end{array}$ & \\
\hline 13 & 1 & 10.7 & 26 & 207 & 62 & -80 & -40 & 170 & 192 & 1 & 106 & 192 & 192 & 192 & 199 & 0.624 & 1.00 \\
\hline 14 & 8 & 10.9 & $\begin{array}{l}-5 \\
-4\end{array}$ & $\begin{array}{l}260 \\
260\end{array}$ & 48 & $\begin{array}{l}-40 \\
-40\end{array}$ & $\begin{array}{l}-220 \\
-220\end{array}$ & $\begin{array}{r}250 \\
250\end{array}$ & $\begin{array}{l}335 \\
335\end{array}$ & 2 & ${ }_{143}^{128}$ & & & & & $\begin{array}{l}1.098 \\
1.545\end{array}$ & \\
\hline 15 & 1 & 10.9 & 1 & 252 & 53 & $\begin{array}{l}-40 \\
-60\end{array}$ & $\begin{array}{l}-220 \\
-180\end{array}$ & $\begin{array}{l}260 \\
250\end{array}$ & $\begin{array}{l}\begin{array}{r}343 \\
314\end{array} \\
3\end{array}$ & $\begin{array}{l}2 \\
2\end{array}$ & $\begin{array}{l}143 \\
124\end{array}$ & 144 & 144 & 145 & 152 & $\begin{array}{l}1.545 \\
0.998\end{array}$ & $\begin{array}{l}0.84 \\
1.00\end{array}$ \\
\hline 16 & 1 & .0 & 68 & 137 & 46 & -190 & 180 & 270 & 376 & 2 & 64 & & & & & 0.137 & 1.00 \\
\hline 17 & $\frac{1}{2}$ & .2 & 4 & 254 & 57 & -60 & -210 & 340 & 404 & 2 & $\begin{array}{c}132 \\
76\end{array}$ & & & & & 1.204 & 1.00 \\
\hline 18 & 2 & 11.2 & 73 & 132 & 42 & -200 & 220 & 270 & 402 & & $\begin{array}{r}10 \\
80\end{array}$ & 84 & 76 & 76 & 84 & 0.264 & 0.90 \\
\hline $\begin{array}{l}19 \\
20\end{array}$ & $\begin{array}{l}1 \\
2\end{array}$ & $\begin{array}{l}11.3 \\
11.3\end{array}$ & $\begin{array}{r}-20 \\
62\end{array}$ & $\begin{array}{l}276 \\
140\end{array}$ & $\begin{array}{l}38 \\
52\end{array}$ & $\begin{array}{r}10 \\
-180\end{array}$ & $\begin{array}{l}-90 \\
150\end{array}$ & $\begin{array}{r}70 \\
300\end{array}$ & $\begin{array}{l}114 \\
381\end{array}$ & $\begin{array}{l}2 \\
2\end{array}$ & $\begin{array}{r}112 \\
88\end{array}$ & & & & & $\begin{array}{l}0.736 \\
0.357\end{array}$ & 1.00 \\
\hline 21 & 2 & 11.4 & 34 & 185 & 71 & & & & & & $\begin{array}{r}92 \\
156\end{array}$ & 97 & 88 & 88 & 97 & 0.414 & 0.91 \\
\hline & & 11.4 & 34 & 180 & 11 & -120 & -10 & 350 & 370 & 2 & $\begin{array}{l}156 \\
158\end{array}$ & 156 & 156 & 164 & 164 & $\begin{array}{l}1.078 \\
2.076\end{array}$ & 0.95 \\
\hline 22 & 2 & 11.5 & 1 & 266 & 58 & -10 & -130 & 210 & 247 & 1 & $\begin{array}{l}62 \\
67\end{array}$ & 70 & 70 & 70 & 77 & $\begin{array}{l}0.125 \\
0.157\end{array}$ & 0.81 \\
\hline 23 & 8 & 11.6 & 15 & 246 & 70 & -40 & -90 & 270 & 87 & 2 & 74 & 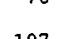 & 1 & 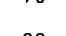 & r & 0. & 0.01 \\
\hline & & & $\begin{array}{r}14 \\
-28\end{array}$ & $\begin{array}{l}252 \\
289\end{array}$ & 71 & $\begin{array}{l}-30 \\
110\end{array}$ & $\begin{array}{r}-90 \\
-320\end{array}$ & $\begin{array}{l}270 \\
220\end{array}$ & 6 & 2 & $\begin{array}{r}92 \\
204\end{array}$ & 107 & 91 & 98 & 112 & $\begin{array}{l}0.406 \\
4.445\end{array}$ & $\begin{array}{l}0.66 \\
1.00\end{array}$ \\
\hline $\begin{array}{l}24 \\
25\end{array}$ & 1 & .9 .9 & $\begin{array}{r}-28 \\
67\end{array}$ & $\begin{array}{l}289 \\
131\end{array}$ & $\begin{array}{l}33 \\
49\end{array}$ & $\begin{array}{r}110 \\
-120\end{array}$ & $\begin{array}{r}320 \\
140\end{array}$ & 210 & $\begin{array}{l}404 \\
279\end{array}$ & 2 & $\begin{array}{r}204 \\
68\end{array}$ & & & & & $\begin{array}{l}.4445 \\
0.165\end{array}$ & $\begin{array}{l}1.00 \\
1.00\end{array}$ \\
\hline 26 & 7 & & $\begin{array}{l}45 \\
39\end{array}$ & 146 & 70 & -60 & 40 & 200 & 13 & 1 & 88 & & & & & 0.357 & 0.62 \\
\hline 27 & 1 & $\begin{array}{l}12.2 \\
12.3\end{array}$ & $\begin{array}{l}39 \\
20\end{array}$ & $\begin{array}{l}153 \\
256\end{array}$ & $\begin{array}{l}76 \\
81\end{array}$ & $\begin{array}{l}-40 \\
-10\end{array}$ & $\begin{array}{r}20 \\
-40\end{array}$ & $\begin{array}{l}180 \\
250\end{array}$ & $\begin{array}{l}185 \\
253\end{array}$ & $\begin{array}{l}1 \\
1\end{array}$ & $\begin{array}{r}113 \\
62\end{array}$ & 117 & 127 & 117 & 141 & 0. & $\begin{array}{l}0.02 \\
1.00\end{array}$ \\
\hline 28 & 1 & 12.3 & $\begin{array}{r}63 \\
-12\end{array}$ & $\begin{array}{l}129 \\
1290\end{array}$ & 53 & -90 & 110 & 190 & 237 & 1 & 86 & & & & & 0 & 1.00 \\
\hline 29 & & 12.6 & -12 & 299 & 50 & 60 & -110 & 150 & 195 & 1 & $\begin{array}{l}116 \\
129\end{array}$ & 128 & 129 & 130 & 133 & $\begin{array}{l}0.817 \\
1.133\end{array}$ & 0.87 \\
\hline 30 & 2 & 12.5 & 2 & 295 & 65 & 60 & -130 & 310 & 341 & 2 & $\begin{array}{l}156 \\
\end{array}$ & & & & & 1.988 & \\
\hline 31 & 9 & 12.8 & 10 & 304 & 73 & 20 & -30 & 120 & 125 & 1 & $\begin{array}{l}157 \\
122\end{array}$ & 156 & 160 & 160 & 164 & $\begin{array}{l}2.026 \\
0.951\end{array}$ & 0.95 \\
\hline 32 & 23 & 12.8 & 19 & 307 & 83 & 30 & -40 & 380 & 383 & 2 & 134 & 143 & 128 & 137 & 146 & $\begin{array}{l}1.262 \\
4.316\end{array}$ & 0.84 \\
\hline$J 2$ & & 12.0 & 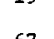 & & 83 & 30 & -40 & 300 & 300 & 2 & 226 & 223 & 230 & 227 & 248 & 6.074 & .81 \\
\hline $\begin{array}{l}33 \\
34\end{array}$ & $\frac{1}{9}$ & $\begin{array}{l}12.8 \\
13.0\end{array}$ & $\begin{array}{l}67 \\
26\end{array}$ & $\begin{array}{r}122 \\
27\end{array}$ & $\begin{array}{l}50 \\
86\end{array}$ & $\begin{array}{r}-120 \\
20\end{array}$ & $\begin{array}{r}190 \\
10\end{array}$ & $\begin{array}{l}270 \\
360\end{array}$ & 1 & $\begin{array}{l}2 \\
2\end{array}$ & $\begin{array}{r}66 \\
194\end{array}$ & & & & & $\begin{array}{l}0.1 \\
3.8\end{array}$ & 1. \\
\hline & & 13.0 & 25 & & 87 & 20 & 0 & 360 & 361 & 2 & 217 & 209 & 239 & 194 & 243 & 5.315 & 0.80 \\
\hline 35 & 3 & 13.1 & 39 & 106 & 78 & -20 & 70 & 330 & 338 & 2 & $\begin{array}{l}196 \\
202\end{array}$ & 210 & 196 & 196 & 210 & $\begin{array}{l}3.9 \\
4.2\end{array}$ & 0.93 \\
\hline 36 & 48 & 12 & $\begin{array}{l}39 \\
35\end{array}$ & 106 & 78 & -20 & 70 & 340 & 348 & 2 & 208 & 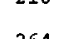 & 200 & & 270 & 4.7 & . \\
\hline 37 & 1 & & $\begin{array}{l}35 \\
77\end{array}$ & $\begin{array}{r}90 \\
122\end{array}$ & 80 & & 6 & $\begin{array}{r}350 \\
80\end{array}$ & & 2 & $\begin{array}{l}247 \\
120\end{array}$ & 264 & 262 & 219 & 275 & & \\
\hline 38 & 1 & 13.3 & 5 & 325 & $\begin{array}{l}67 \\
67\end{array}$ & 130 & $\begin{array}{l}-90 \\
-90\end{array}$ & 370 & $\begin{array}{l}124 \\
402\end{array}$ & 1 & $\begin{array}{l}120 \\
164\end{array}$ & & & & & 2.3 & $\begin{array}{l}1.00 \\
1.00\end{array}$ \\
\hline 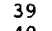 & 1 & 13.6 & -10 & 321 & 50 & 200 & -160 & 310 & 402 & 2 & 158 & & & & & 2.0 & 1.0 \\
\hline 40 & 2 & 14.0 & 58 & 106 & 57 & -40 & 140 & 220 & 264 & 1 & $\begin{array}{l}108 \\
113\end{array}$ & 116 & 108 & 108 & 116 & $\begin{array}{l}0.6 \\
0.7\end{array}$ & 0.93 \\
\hline 41 & 8 & 14.2 & -8 & 336 & 49 & 200 & -90 & 250 & 333 & 2 & $\begin{array}{l}162 \\
162\end{array}$ & 110 & 108 & 100 & & & ד. \\
\hline 42 & 12 & & & & & & -90 & & & & 178 & 184 & 184 & 174 & 198 & 2. & 0.82 \\
\hline & & & -7 & 6 & 50 & 200 & -90 & 26 & & 2 & $\begin{array}{l}166 \\
192\end{array}$ & 198 & 213 & 174 & 221 & & 0.75 \\
\hline 43 & 32 & & 5 & 354 & 58 & 18 & -20 & 29 & 3 & & 160 & & & & & & \\
\hline & & & 7 & 357 & 59 & & -1 & & & & 194 & 199 & 202 & 200 & 221 & & 0.72 \\
\hline 44 & 11 & & $\begin{array}{l}6 \\
6\end{array}$ & 355 & $\begin{array}{l}59 \\
58\end{array}$ & & -1 & & & & 94 & 112 & 138 & 124 & 145 & & 0.65 \\
\hline 45 & 1 & & -1 & $\begin{array}{l}355 \\
348\end{array}$ & $\begin{array}{l}58 \\
52\end{array}$ & $\begin{array}{l}1 \\
1\end{array}$ & $\begin{array}{l}-1 \\
-3\end{array}$ & & & & $\begin{array}{r}120 \\
94\end{array}$ & 112 & 200 & 14 & 145 & & 1.00 \\
\hline 4 & 8 & & $\begin{array}{l}-12 \\
-11\end{array}$ & $\begin{array}{l}340 \\
339\end{array}$ & $\begin{array}{l}42 \\
44\end{array}$ & $\begin{array}{l}280 \\
260\end{array}$ & $\begin{array}{l}-100 \\
-100\end{array}$ & 27 & $4 c$ & 2 & $\begin{array}{l}16 \\
18\end{array}$ & 201 & 169 & 172 & 201 & & \\
\hline 47 & 1 & & -12 & 339 & 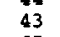 & 260 & -100 & 2 & & & 18 & 201 & 107 & 172 & 200 & & 1.00 \\
\hline 48 & 10 & & 38 & 66 & 65 & 0 & 9 & & & & 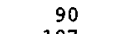 & & & & & & \\
\hline & & & 37 & & 66 & & & & & 1 & 15 & 98 & 117 & 109 & 122 & & 0.74 \\
\hline 49 & 1 & & 70 & 110 & 44 & -100 & & & & & & & & & & & 1. \\
\hline 50 & 11 & & $\begin{array}{l}43 \\
44\end{array}$ & $\begin{array}{l}74 \\
75\end{array}$ & $\begin{array}{l}60 \\
58\end{array}$ & $\begin{array}{l}50 \\
50\end{array}$ & $\begin{array}{l}18 \\
19\end{array}$ & $\begin{array}{l}32 \\
32\end{array}$ & $\begin{array}{l}37 \\
37\end{array}$ & & $\begin{array}{l}17 \\
15\end{array}$ & 181 & 209 & 181 & 211 & & 0.82 \\
\hline 51 & 20 & 15 & 44 & 73 & 57 & 60 & 200 & 320 & 382 & 2 & $\begin{array}{l}178 \\
205\end{array}$ & 199 & 210 & 213 & 221 & & \\
\hline $\begin{array}{l}52 \\
53\end{array}$ & $\begin{array}{l}1 \\
1\end{array}$ & & $\begin{array}{l}66 \\
44\end{array}$ & $\begin{array}{r}103 \\
71\end{array}$ & $\begin{array}{l}44 \\
47\end{array}$ & & & & & 2 & 12 & & & & & & \\
\hline 54 & 3 & & $\begin{array}{l}44 \\
24\end{array}$ & 42 & 42 & & $\begin{array}{l}26 \\
2 C\end{array}$ & & & & $\begin{array}{l}15 \\
20\end{array}$ & & & & & $\begin{array}{l}1.9 \\
4.4\end{array}$ & 1. \\
\hline 55 & 3 & & $\begin{array}{r}25 \\
5\end{array}$ & $\begin{array}{l}44 \\
21\end{array}$ & $\begin{array}{l}42 \\
33\end{array}$ & 260 & 21 & 2 & & & 21 & 214 & 214 & 213 & 218 & 2 & 0.94 \\
\hline ה נת & J & & & & & & 100 & $18 \mathrm{C}$ & 332 & & $\begin{array}{l}21 \\
22\end{array}$ & 224 & 225 & 216 & 227 & 5 & 0.95 \\
\hline 56 & 5 & 16.6 & 49 & 76 & 41 & 50 & 200 & 180 & 274 & 2 & & 150 & 162 & 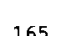 & . & & \\
\hline 57 & 1 & 1 & 75 & 108 & 34 & -60 & 180 & 130 & & 2 & 102 & 200 & 200 & & & 6 & 1. \\
\hline 5 & 18 & 16.8 & 63 & 94 & 37 & -20 & 260 & 200 & 329 & 2 & $\begin{array}{l}16 \\
18\end{array}$ & 181 & 182 & 181 & 197 & & 0.82 \\
\hline 59 & 33 & 1 & 56 & $\begin{array}{l}85 \\
90\end{array}$ & 37 & 10 & $\begin{array}{l}120 \\
120\end{array}$ & & & 2 & $\begin{array}{l}134 \\
171\end{array}$ & 158 & 182 & 186 & 197 & 2.598 & \\
\hline 60 & 1 & $\begin{array}{l}16.2 \\
17.4\end{array}$ & $\begin{array}{l}39 \\
48\end{array}$ & $\begin{array}{l}90 \\
75\end{array}$ & $\begin{array}{l}43 \\
33\end{array}$ & 60 & $\begin{array}{l}120 \\
220\end{array}$ & $\begin{array}{l}110 \\
150\end{array}$ & $\begin{array}{l}163 \\
273 \\
\end{array}$ & & 146 & & & & & 1.630 & 1.00 \\
\hline
\end{tabular}


Table 4. Voids of $R \geq 0 \mathrm{~A} / \mathrm{ACO}$ clusters (sample A/L, $p$-method)

\begin{tabular}{|c|c|c|c|c|c|c|c|c|c|c|c|c|c|c|c|c|c|}
\hline No. & $\mathrm{n}_{\mathrm{cg}}$ & $\begin{array}{r}\alpha \\
\alpha_{c} \\
{[h]}\end{array}$ & $\begin{array}{r}\delta \\
\delta_{c} \\
{\left[{ }^{\circ}\right]}\end{array}$ & $\begin{array}{r}1 \\
10 \\
{\left[{ }_{0}^{\circ}\right]} \\
\end{array}$ & $\begin{array}{l}b \\
b \circ \\
\left.b_{0}^{\circ}\right]\end{array}$ & $\begin{array}{l}x \\
x_{e}\end{array}$ & $\begin{array}{c}y \\
y_{c} \\
{\left[h^{-1} \mathrm{Mpc}\right]} \\
\end{array}$ & $\begin{array}{l}z \\
z_{c}\end{array}$ & $\begin{array}{c}r \\
r_{\mathrm{c}} \\
{\left[h^{-1} \mathrm{Mpc}\right]} \\
\end{array}$ & $\begin{array}{l}\text { SV } \\
\mathrm{SV}_{\mathrm{c}}\end{array}$ & $\begin{array}{c}D \\
D \\
{\left[h^{-1} \mathrm{Mpc}\right]}\end{array}$ & $\begin{array}{c}D_{x} \\
\quad[h \\
\end{array}$ & $\begin{array}{c}D_{y} \\
-1 \\
-1 \\
\end{array}$ & $D_{z}$ & $D_{\max }$ & $\begin{array}{c}V \\
V_{\mathrm{T}} \\
{\left[10^{6} h^{-3} \mathrm{Mpc}^{3}\right]}\end{array}$ & $\begin{array}{l}s \\
s\end{array}$ \\
\hline 1 & 7 & $\begin{array}{l}8.0 \\
8.3\end{array}$ & 52 & 166 & 33 & $\begin{array}{l}-240 \\
\end{array}$ & 60 & 160 & 295 & 2 & 128 & 145 & & & 140 & 1.098 & \\
\hline 2 & 1 & $\begin{array}{l}8.3 \\
8.3\end{array}$ & $\begin{array}{l}50 \\
50\end{array}$ & $\begin{array}{l}168 \\
169\end{array}$ & $\begin{array}{l}\begin{array}{l}35 \\
35\end{array} \\
\text { - }\end{array}$ & $\begin{array}{l}-240 \\
-210\end{array}$ & $\begin{array}{l}50 \\
40\end{array}$ & $\begin{array}{l}170 \\
150\end{array}$ & $\begin{array}{l}298 \\
261 \\
261\end{array}$ & $\begin{array}{l}2 \\
2\end{array}$ & $\begin{array}{l}143 \\
122\end{array}$ & 145 & 137 & 145 & 149 & $\begin{array}{l}1.519 \\
0.951\end{array}$ & $\begin{array}{l}0.86 \\
1.00\end{array}$ \\
\hline $\begin{array}{l}3 \\
4\end{array}$ & $\frac{1}{7}$ & $\begin{array}{l}8.6 \\
8.9\end{array}$ & $\begin{array}{c}65 \\
8\end{array}$ & $\begin{array}{l}150 \\
220\end{array}$ & $\begin{array}{l}36 \\
33\end{array}$ & $\begin{array}{l}-120 \\
-200\end{array}$ & $\begin{array}{r}70 \\
-170\end{array}$ & 100 & $\begin{array}{l}171 \\
313\end{array}$ & $\frac{2}{2}$ & 94 & & & & & 0.435 & 1.00 \\
\hline & & 9.0 & 8 & 222 & 34 & -190 & -170 & 170 & 306 & 2 & 145 & 147 & 154 & 138 & 160 & 1.586 & 0.81 \\
\hline 5 & 12 & $\begin{array}{l}8.9 \\
8.9\end{array}$ & $\begin{array}{l}65 \\
67\end{array}$ & $\begin{array}{l}149 \\
148\end{array}$ & $\begin{array}{l}38 \\
37\end{array}$ & $\begin{array}{l}-220 \\
-220\end{array}$ & $\begin{array}{l}130 \\
140\end{array}$ & $\begin{array}{l}200 \\
200\end{array}$ & $\begin{array}{l}324 \\
329\end{array}$ & $\begin{array}{l}2 \\
2\end{array}$ & $\begin{array}{l}110 \\
130\end{array}$ & 126 & 133 & 126 & 140 & $\begin{array}{l}0.697 \\
1.155\end{array}$ & 0.79 \\
\hline 6 & 4 & 9.1 & 30 & 196 & 43 & -280 & -80 & 270 & 397 & 2 & 182 & 190 & 190 & 199 & 203 & 3.157 & 09 \\
\hline 7 & 3 & 9.1 & 68 & 145 & 38 & -160 & 110 & 150 & 245 & 2 & $\begin{array}{r}92 \\
64\end{array}$ & 190 & 190 & 199 & 203 & $\begin{array}{l}3.730 \\
0.137\end{array}$ & 0.90 \\
\hline 8 & 4 & $\begin{array}{l}9.2 \\
9.5\end{array}$ & $\begin{array}{l}36 \\
36\end{array}$ & $\begin{array}{l}187 \\
187\end{array}$ & $\begin{array}{l}45 \\
48\end{array}$ & $\begin{array}{l}-80 \\
-80\end{array}$ & $\begin{array}{l}-10 \\
-10\end{array}$ & $\begin{array}{l}80 \\
90\end{array}$ & $\begin{array}{l}114 \\
121\end{array}$ & 1 & $\begin{array}{l}73 \\
88 \\
97\end{array}$ & $\begin{array}{l}73 \\
88\end{array}$ & $\begin{array}{l}73 \\
98\end{array}$ & $\begin{array}{r}72 \\
105\end{array}$ & $\begin{array}{r}78 \\
707\end{array}$ & $\begin{array}{l}0.201 \\
0.357 \\
0.487\end{array}$ & 0.82 \\
\hline 9 & 14 & 9.4 & 13 & 219 & 41 & -100 & -80 & 110 & 169 & 1 & 106 & & & 100 & 107 & 0.624 & 0.82 \\
\hline & 9 & 9.7 & 21 & 211 & 48 & -100 & -60 & 130 & 175 & 1 & 136 & 113 & 161 & 146 & 168 & 1.323 & 0.63 \\
\hline 10 & 9 & $\begin{array}{l}9.5 \\
9.3\end{array}$ & $\begin{array}{l}51 \\
51\end{array}$ & $\begin{array}{l}166 \\
167\end{array}$ & $\begin{array}{l}46 \\
44\end{array}$ & $\begin{array}{l}-160 \\
-170\end{array}$ & $\begin{array}{l}40 \\
40\end{array}$ & $\begin{array}{l}170 \\
170\end{array}$ & $\begin{array}{l}237 \\
244\end{array}$ & $\frac{1}{1}$ & 126 & 134 & 134 & 142 & 115 & 1.047 & 0.87 \\
\hline 11 & 3 & 9.6 & & 191 & $\begin{array}{l}449 \\
49\end{array}$ & $\begin{array}{l}-170 \\
-160\end{array}$ & $\begin{array}{l}40 \\
-30\end{array}$ & 190 & 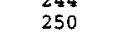 & $\frac{1}{1}$ & $\begin{array}{l}140 \\
102\end{array}$ & 134 & 134 & 142 & 145 & $\begin{array}{l}1.429 \\
0.556\end{array}$ & 0.87 \\
\hline 12 & 8 & $\begin{array}{l}9.5 \\
9.7\end{array}$ & $\begin{array}{l}34 \\
24\end{array}$ & $\begin{array}{l}191 \\
207\end{array}$ & $\begin{array}{l}48 \\
48\end{array}$ & $\begin{array}{l}-160 \\
-180\end{array}$ & $\begin{array}{l}-30 \\
-90\end{array}$ & $\begin{array}{l}180 \\
220\end{array}$ & $\begin{array}{l}243 \\
298\end{array}$ & $\frac{1}{2}$ & $\begin{array}{l}108 \\
120\end{array}$ & 110 & 102 & 110 & 114 & 0.661 & 0.89 \\
\hline 13 & 1 & & T & 107 & & & 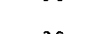 & & & & 131 & 129 & 136 & 128 & 139 & $\begin{array}{l}1.185 \\
\end{array}$ & 0.86 \\
\hline 14 & $\frac{1}{5}$ & $\begin{array}{l}9.8 \\
9.8\end{array}$ & $\begin{array}{l}30 \\
42\end{array}$ & $\begin{array}{l}197 \\
178\end{array}$ & $\begin{array}{l}51 \\
50\end{array}$ & $\begin{array}{l}-100 \\
-260\end{array}$ & $\begin{array}{r}-30 \\
10\end{array}$ & $\begin{array}{l}130 \\
310\end{array}$ & $\begin{array}{l}167 \\
405\end{array}$ & $\frac{1}{2}$ & $\begin{array}{r}98 \\
734\end{array}$ & & & & & 0.493 & 1.00 \\
\hline 15 & 7 & 9.6 & 44 & 176 & 49 & -260 & 20 & 300 & $\begin{array}{l}407 \\
397\end{array}$ & 2 & $\begin{array}{l}145 \\
145\end{array}$ & 146 & 149 & 141 & 155 & $\begin{array}{l}1.500 \\
1.589\end{array}$ & 0.86 \\
\hline 13 & & 9.7 & 53 & $\begin{array}{l}162 \\
162\end{array}$ & $\begin{array}{l}49 \\
48\end{array}$ & $\begin{array}{l}-190 \\
-190\end{array}$ & $\begin{array}{l}60 \\
60\end{array}$ & 230 & 304 & 2 & 132 & & & & & 1.204 & \\
\hline 16 & 1 & 10.0 & 1 & 239 & 42 & -90 & $\begin{array}{r}00 \\
-150\end{array}$ & 160 & $\begin{array}{l}297 \\
237\end{array}$ & $\begin{array}{l}2 \\
1\end{array}$ & $\begin{array}{r}146 \\
58\end{array}$ & 147 & 140 & 155 & 158 & $\begin{array}{l}1.618 \\
0.102\end{array}$ & $\begin{array}{l}0.84 \\
1.00\end{array}$ \\
\hline 17 & 2 & 10.0 & 13 & 225 & 49 & -110 & -110 & 180 & 238 & 1 & 78 & 78 & 86 & 78 & 86 & 0.248 & 1.00 \\
\hline 18 & 3 & 10.1 & 70 & 139 & 41 & -70 & 60 & 80 & 122 & 1 & 92 & 18 & 86 & 18 & 86 & 0.408 & 0.91 \\
\hline 19 & & & 66 & 144 & 43 & -70 & 50 & 80 & 117 & 1 & 102 & 99 & 109 & 99 & 113 & 0.551 & 0.81 \\
\hline $\begin{array}{l}19 \\
20\end{array}$ & $\begin{array}{l}1 \\
1\end{array}$ & $\begin{array}{l}10.2 \\
10.3\end{array}$ & $\begin{array}{r}14 \\
-16\end{array}$ & $\begin{array}{l}225 \\
260\end{array}$ & $\begin{array}{l}52 \\
33\end{array}$ & $\begin{array}{l}-100 \\
-60\end{array}$ & $\begin{array}{l}-100 \\
-330\end{array}$ & $\begin{array}{l}180 \\
220\end{array}$ & $\begin{array}{l}229 \\
401\end{array}$ & $\frac{1}{2}$ & $\begin{array}{r}80 \\
156\end{array}$ & & & & & 0.268 & 1.00 \\
\hline 21 & 6 & 10.4 & 15 & 225 & 56 & $\begin{array}{l}-00 \\
-160\end{array}$ & $\begin{array}{l}-350 \\
-160\end{array}$ & 330 & 400 & 2 & $\begin{array}{l}156 \\
180\end{array}$ & & & & & $\begin{array}{l}1.988 \\
3.054\end{array}$ & \\
\hline $\begin{array}{l}22 \\
23\end{array}$ & $\frac{1}{7}$ & $\begin{array}{l}10.8 \\
10.9\end{array}$ & $\begin{array}{r}3 \\
62\end{array}$ & $\begin{array}{l}248 \\
143\end{array}$ & 52 & $\begin{array}{r}-40 \\
-200\end{array}$ & -100 & 140 & 177 & 1 & $\begin{array}{r}189 \\
68\end{array}$ & 185 & 194 & 190 & 199 & $\begin{array}{l}3.543 \\
0.165\end{array}$ & $\begin{array}{l}0.90 \\
1.00\end{array}$ \\
\hline & & & & 143 & 50 & -200 & 150 & 300 & 391 & 2 & $\begin{array}{l}76 \\
92\end{array}$ & 101 & 92 & 94 & 108 & $\begin{array}{l}0.230 \\
0.406\end{array}$ & 0.70 \\
\hline $\begin{array}{l}24 \\
25\end{array}$ & $\begin{array}{l}1 \\
5\end{array}$ & $\begin{array}{l}11.0 \\
11.0\end{array}$ & $\begin{array}{r}-19 \\
28\end{array}$ & $\begin{array}{l}270 \\
205\end{array}$ & $\begin{array}{l}36 \\
66\end{array}$ & $\begin{array}{r}0 \\
-130\end{array}$ & $\begin{array}{r}-110 \\
-60\end{array}$ & $\begin{array}{r}80 \\
320\end{array}$ & $\begin{array}{l}136 \\
351\end{array}$ & $\begin{array}{l}2 \\
2\end{array}$ & & & & & & $\begin{array}{l}0.333 \\
1.204\end{array}$ & 1.00 \\
\hline & & 11.0 & $\begin{array}{l}20 \\
29\end{array}$ & 201 & $\begin{array}{l}00 \\
66\end{array}$ & $\begin{array}{l}-130 \\
-130\end{array}$ & $\begin{array}{l}-60 \\
-50\end{array}$ & $\begin{array}{l}320 \\
320\end{array}$ & $\begin{array}{l}351 \\
349\end{array}$ & $\begin{array}{l}2 \\
2\end{array}$ & $\begin{array}{l}132 \\
142\end{array}$ & 132 & 148 & 142 & 150 & $\begin{array}{l}1.204 \\
1.499\end{array}$ & 0.88 \\
\hline 26 & 1 & 11.0 & 68 & 137 & 46 & -190 & 180 & 270 & 376 & 2 & 64 & & & & & 0.137 & \\
\hline 27 & 1 & & 4 & 254 & 57 & -60 & -210 & 340 & 404 & 2 & 132 & & & & & & 00 \\
\hline $\begin{array}{l}28 \\
29\end{array}$ & $\begin{array}{l}1 \\
4\end{array}$ & 1 & 11 & 243 & 62 & -50 & -100 & 210 & 238 & 1 & 56 & & & & & 0.092 & 1.00 \\
\hline 29 & 4 & $\begin{array}{l}11.1 \\
11.1\end{array}$ & $\begin{array}{l}15 \\
12\end{array}$ & $\begin{array}{l}236 \\
240\end{array}$ & $\begin{array}{l}64 \\
62\end{array}$ & $\begin{array}{l}-40 \\
-40\end{array}$ & $\begin{array}{l}-60 \\
-70\end{array}$ & $\begin{array}{l}150 \\
150\end{array}$ & $\begin{array}{l}166 \\
170\end{array}$ & $\begin{array}{l}1 \\
1\end{array}$ & $\begin{array}{l}72 \\
80\end{array}$ & 80 & 89 & 80 & 89 & $\begin{array}{l}0.3 \\
0.2\end{array}$ & 0.81 \\
\hline $\begin{array}{l}30 \\
31\end{array}$ & $\begin{array}{r}1 \\
11\end{array}$ & $\begin{array}{l}11.2 \\
11.4\end{array}$ & $\begin{array}{r}55 \\
5\end{array}$ & $\begin{array}{l}147 \\
257\end{array}$ & $\begin{array}{l}57 \\
60\end{array}$ & $\begin{array}{r}-170 \\
-20\end{array}$ & $\begin{array}{r}110 \\
-90\end{array}$ & 310 & 370 & 2 & $\begin{array}{l}76 \\
72\end{array}$ & & & & & 0.195 & 1.00 \\
\hline & & & 7 & 256 & 63 & $\begin{array}{l}-20 \\
-20\end{array}$ & -80 & $\begin{array}{l}160 \\
160\end{array}$ & $\begin{array}{l}180 \\
180\end{array}$ & 1 & 96 & 87 & 126 & 80 & 130 & 0.464 & 0.55 \\
\hline 32 & 2 & 11.4 & 23 & 220 & 70 & -60 & -50 & 210 & 224 & 1 & 72 & & & & & 0.195 & \\
\hline 33 & 5 & $\begin{array}{l}11.4 \\
11.6\end{array}$ & $\begin{array}{l}23 \\
15\end{array}$ & $\begin{array}{l}220 \\
246\end{array}$ & $\begin{array}{l}70 \\
70\end{array}$ & $\begin{array}{l}-60 \\
-40\end{array}$ & $\begin{array}{l}-50 \\
-90\end{array}$ & 220 & 233 & $\frac{1}{2}$ & $\begin{array}{l}76 \\
74\end{array}$ & 82 & 72 & 82 & 86 & 0.234 & 0.84 \\
\hline & & 11.6 & & & & -40 & -90 & 270 & 287 & 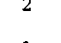 & $\begin{array}{l}14 \\
83\end{array}$ & 89 & 84 & 82 & 89 & $\begin{array}{l}0.212 \\
0.304\end{array}$ & 0.83 \\
\hline 34 & 3 & $\begin{array}{l}11.8 \\
11.9\end{array}$ & $\begin{array}{l}3 \\
5\end{array}$ & $\begin{array}{l}270 \\
270\end{array}$ & $\begin{array}{l}62 \\
65\end{array}$ & $\begin{array}{l}0 \\
0\end{array}$ & $\begin{array}{l}-90 \\
-80\end{array}$ & $\begin{array}{l}170 \\
170\end{array}$ & $\begin{array}{l}192 \\
188\end{array}$ & $\begin{array}{l}1 \\
1\end{array}$ & $\begin{array}{l}76 \\
82\end{array}$ & 83 & 83 & 76 & 87 & 0285 & 87 \\
\hline 35 & 1 & 1. & -28 & 289 & 33 & 110 & $\begin{array}{r}-00 \\
-320\end{array}$ & 220 & $\begin{array}{l}100 \\
404\end{array}$ & $\frac{1}{2}$ & $\begin{array}{r}204 \\
204\end{array}$ & & & & & 4.4 & \\
\hline 36 & $\frac{1}{3}$ & & 22 & 233 & 77 & & -40 & 220 & 2 & 1 & 66 & & & & & & 1.00 \\
\hline 37 & 3 & 11.9 & 67 & 131 & 49 & -120 & 140 & 210 & 279 & 2 & 68 & & & & & 0.1 & 0 \\
\hline 38 & 4 & $\begin{array}{l}11.6 \\
12.0\end{array}$ & $\begin{array}{l}68 \\
69\end{array}$ & $\begin{array}{l}133 \\
129\end{array}$ & $\begin{array}{l}48 \\
47\end{array}$ & $\begin{array}{l}-130 \\
-170\end{array}$ & $\begin{array}{l}140 \\
210\end{array}$ & $\begin{array}{l}210 \\
290\end{array}$ & $\begin{array}{l}284 \\
396\end{array}$ & 2 & $\begin{array}{l}74 \\
66\end{array}$ & 76 & 68 & 76 & 80 & 0.2 & 0.85 \\
\hline 39 & & & -13 & 288 & 48 & 20 & & $\begin{array}{r}290 \\
70\end{array}$ & 396 & 2 & $\begin{array}{l}60 \\
78 \\
78\end{array}$ & 75 & 84 & 66 & 86 & 0.2 & 0.77 \\
\hline 40 & 4 & 12.1 & 22 & 236 & $\begin{array}{l}80 \\
79\end{array}$ & -20 & -30 & 190 & $\begin{array}{r}34 \\
193\end{array}$ & 1 & $\begin{array}{l}66 \\
66\end{array}$ & & & & & & \\
\hline 41 & 1 & 12.2 & 38 & 1 & 7 & -60 & 30 & 300 & 307 & 2 & 74 & 74 & 66 & 76 & 78 & 0.2 & 0.85 \\
\hline 42 & 1 & & -13 & 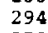 & 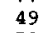 & 50 & -1 & $\begin{array}{l}140 \\
140\end{array}$ & & $\begin{array}{l}2 \\
1\end{array}$ & $\begin{array}{l}150 \\
100\end{array}$ & & & & & & \\
\hline 43 & 1 & 12.4 & 11 & $\therefore$ & 13 & 10 & -60 & 20 & & 1 & 62 & & & & & 0. & \\
\hline $\begin{array}{l}44 \\
45\end{array}$ & $\frac{1}{2}$ & & 22 & 252 & 83 & -10 & -30 & 250 & & 1 & & & & & & & 1.00 \\
\hline & 2 & 12.5 & & 294 & 58 & 70 & -160 & 280 & $3=$ & 2 & $\begin{array}{l}126 \\
130\end{array}$ & 126 & 136 & 126 & 136 & & 0.93 \\
\hline 46 & 5 & 12.5 & 37 & 146 & 79 & -30 & 20 & 190 & 19 & 1 & 74 & & & & & 0.2 & \\
\hline 47 & 8 & & & & . & & 10 & 190 & 19 & 1 & 11 & 102 & 84 & 82 & 105 & & 0.70 \\
\hline 41 & 8 & 12.6 & -12 & 299 & 50 & 60 & -110 & 150 & 195 & 1 & 10 & 120 & 122 & 114 & 125 & & 0.86 \\
\hline 48 & 27 & 12.6 & $\begin{array}{l}-2 \\
-7\end{array}$ & $\begin{array}{r}295 \\
295\end{array}$ & 60 & 70 & $\begin{array}{l}-150 \\
-150\end{array}$ & 290 & $\begin{array}{l}334 \\
343\end{array}$ & 2 & & & & & & & \\
\hline 49 & 3 & $\begin{array}{l}12.5 \\
12.7\end{array}$ & $\begin{array}{l}-1 \\
-6\end{array}$ & $\begin{array}{l}295 \\
301\end{array}$ & 56 & $\begin{array}{r}70 \\
110\end{array}$ & $\begin{array}{l}-150 \\
-180\end{array}$ & $\begin{array}{l}300 \\
310\end{array}$ & $\begin{array}{l}\begin{array}{l}343 \\
375\end{array} \\
375\end{array}$ & $\begin{array}{l}2 \\
2\end{array}$ & $\begin{array}{l}1 \\
1\end{array}$ & 186 & 161 & 168 & 204 & 1.3 & 0.68 \\
\hline 50 & 1 & & 5 & & 69 & & & & & & 144 & 144 & 144 & 146 & 149 & & 0.93 \\
\hline 51 & 30 & & $\begin{array}{l}5 \\
41 \\
\end{array}$ & & 7 & & $\begin{array}{r}-80 \\
70\end{array}$ & $\begin{array}{l}24 \\
32\end{array}$ & $\begin{array}{l}25 \\
33 \\
-12\end{array}$ & $\begin{array}{l}\frac{1}{2} \\
\text { L }\end{array}$ & 16 & & & & & & 1.00 \\
\hline & & & & & & -40 & & 3 & & & & 182 & 216 & 200 & 220 & & 0.75 \\
\hline $\begin{array}{l}52 \\
53\end{array}$ & $\begin{array}{l}1 \\
5\end{array}$ & 12.8 & $\begin{array}{l}45 \\
45\end{array}$ & 121 & $\begin{array}{l}72 \\
72\end{array}$ & -60 & 100 & $\begin{array}{l}360 \\
380\end{array}$ & 400 & 2 & & & & & & 2 & 1.00 \\
\hline & & & & & & & & & & & $\begin{array}{l}168 \\
178\end{array}$ & 192 & 174 & 168 & 192 & 2. & 0. \\
\hline $\begin{array}{l}34 \\
55\end{array}$ & $\begin{array}{l}1 \\
1\end{array}$ & & $\overline{1}$ & 30 & 5 & $\begin{array}{r}440 \\
50\end{array}$ & $\begin{array}{l}-180 \\
-50\end{array}$ & $\begin{array}{l}33 \\
40\end{array}$ & 40 & 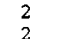 & 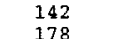 & & & & & & \\
\hline 56 & 8 & & 24 & & 8 & 10 & 0 & & & & & & & & & & \\
\hline & & & 1 & 33 & 7 & & -10 & & & 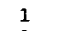 & 128 & 128 & 143 & 118 & 149 & 1.091 & 0 \\
\hline 57 & 1 & & 20 & & 8 & & & & & & & & & & & & \\
\hline $\begin{array}{l}58 \\
59\end{array}$ & $\begin{array}{r}1 \\
12\end{array}$ & & $\begin{array}{l}-12 \\
-12\end{array}$ & 313 & $\begin{array}{l}50 \\
50\end{array}$ & $\begin{array}{l}170 \\
180\end{array}$ & $\begin{array}{l}-180 \\
-180\end{array}$ & $\begin{array}{l}25 \\
30 \\
30\end{array}$ & & 2 & $\begin{array}{l}142 \\
152\end{array}$ & & & & & 18 & 1. \\
\hline & & & -11 & & S & & & 30 & & 2 & $\begin{array}{l}172 \\
172\end{array}$ & 178 & 183 & 159 & 190 & $\begin{array}{l}\frac{1.8}{2.6} \\
2.6\end{array}$ & \\
\hline 60 & 1 & & 42 & 10 & 7 & -20 & 1 & 3 & 365 & 2 & 160 & & & & & 2. & 1.00 \\
\hline 61 & 1 & & 6 & & 67 & 130 & -90 & 37 & 40 & 2 & 164 & & & & & 2.310 & 1.00 \\
\hline 62 & 2 & 13.4 & 16 & 338 & 76 & 50 & -20 & 220 & 226 & 1 & $\begin{array}{l}84 \\
88\end{array}$ & 93 & 93 & 84 & 97 & $\begin{array}{l}0.310 \\
0.358\end{array}$ & 0.87 \\
\hline
\end{tabular}


Table 4. continued

\begin{tabular}{|c|c|c|c|c|c|c|c|c|c|c|c|c|c|c|c|c|c|}
\hline No. & $n_{e s}$ & $\begin{array}{c}\alpha \\
\alpha_{c} \\
{[h]}\end{array}$ & $\begin{array}{c}\delta \\
\delta_{c} \\
{\left[{ }^{\circ}\right]}\end{array}$ & $\begin{array}{l}1 \\
10 \\
10\end{array}$ & $\begin{array}{l}b \\
b_{c} \\
{[0]}\end{array}$ & $\begin{array}{l}x \\
x_{c} \\
\end{array}$ & $\begin{array}{c}y \\
y_{\mathrm{c}} \\
h^{-1} \mathrm{Mpc}\end{array}$ & $\begin{array}{l}z_{c} \\
z_{c}\end{array}$ & $\begin{array}{c}r \\
r_{c} \\
{\left[h^{-1} \mathrm{Mpc}\right]}\end{array}$ & $\begin{array}{l}\mathrm{SV} \\
\mathrm{SV}_{\mathrm{c}}\end{array}$ & $\begin{array}{c}D \\
D_{\mathrm{e}} \\
{\left[\boldsymbol{h}^{-1} \mathrm{Mpc}\right]}\end{array}$ & $\begin{array}{c}D_{x} \\
\quad[h \\
\end{array}$ & $\begin{array}{c}D_{y} \\
-1 \quad \mathrm{Mpc} \\
\end{array}$ & $D_{z}$ & $D_{\max }$ & $\begin{array}{c}V \\
V_{\uparrow} \\
{\left[10^{6} h^{-3} \mathrm{Mpc}^{3}\right]}\end{array}$ & $\begin{array}{l}s \\
s\end{array}$ \\
\hline $\begin{array}{l}63 \\
64\end{array}$ & $\begin{array}{l}1 \\
1\end{array}$ & $\begin{array}{l}13.5 \\
13.7\end{array}$ & $\begin{array}{r}9 \\
-22\end{array}$ & $\begin{array}{l}333 \\
319\end{array}$ & $\begin{array}{l}70 \\
39\end{array}$ & $\begin{array}{r}60 \\
150\end{array}$ & $\begin{array}{r}-30 \\
-130\end{array}$ & $\begin{array}{l}180 \\
160\end{array}$ & $\begin{array}{l}192 \\
255\end{array}$ & $\frac{1}{2}$ & $\begin{array}{l}74 \\
86\end{array}$ & & & & & $\begin{array}{l}0.212 \\
0.333 \\
0.373\end{array}$ & $\begin{array}{l}1.00 \\
1.00\end{array}$ \\
\hline & & & & & & & & & & & $\begin{array}{l}138 \\
144\end{array}$ & 147 & 147 & 138 & 151 & $\begin{array}{l}1.376 \\
1.560\end{array}$ & 0.91 \\
\hline 66 & 5 & $\begin{array}{l}13.8 \\
13.6\end{array}$ & $\begin{array}{r}-9 \\
-11\end{array}$ & $\begin{array}{l}326 \\
323\end{array}$ & $\begin{array}{l}50 \\
49\end{array}$ & $\begin{array}{l}210 \\
200\end{array}$ & $\begin{array}{l}-140 \\
-150\end{array}$ & $\begin{array}{l}300 \\
290\end{array}$ & $\begin{array}{l}392 \\
383\end{array}$ & $\begin{array}{l}2 \\
2\end{array}$ & $\begin{array}{l}150 \\
160\end{array}$ & 158 & 165 & 165 & 169 & $\begin{array}{l}1.767 \\
2.146\end{array}$ & 0.89 \\
\hline $\begin{array}{l}67 \\
68\end{array}$ & $\begin{array}{l}1 \\
2\end{array}$ & $\begin{array}{l}13.8 \\
14.0\end{array}$ & $\begin{array}{l}57 \\
58\end{array}$ & $\begin{array}{l}108 \\
106\end{array}$ & $\begin{array}{l}58 \\
57\end{array}$ & $\begin{array}{l}-60 \\
-40\end{array}$ & $\begin{array}{l}190 \\
140\end{array}$ & $\begin{array}{l}320 \\
220\end{array}$ & $\begin{array}{l}377 \\
264\end{array}$ & $\begin{array}{l}2 \\
1\end{array}$ & $\begin{array}{r}50 \\
100\end{array}$ & & & & & $\begin{array}{l}0.065 \\
0.524\end{array}$ & 1.00 \\
\hline 69 & 2 & 14.1 & 59 & 105 & 55 & -60 & 220 & 320 & 393 & 2 & $\begin{array}{r}105 \\
56\end{array}$ & 109 & 109 & 100 & 113 & $\begin{array}{l}0.608 \\
0.092\end{array}$ & 0.88 \\
\hline 70 & 37 & 14.2 & -6 & 338 & 50 & 200 & -80 & 260 & 338 & 2 & $\begin{array}{r}58 \\
164\end{array}$ & 56 & 56 & 65 & 65 & $\begin{array}{l}0.102 \\
2.310\end{array}$ & 0.86 \\
\hline 71 & 4 & 14 & 1 & $\begin{array}{r}348 \\
345\end{array}$ & 55 & $\begin{array}{l}190 \\
170\end{array}$ & $\begin{array}{l}-40 \\
-30\end{array}$ & $\begin{array}{l}280 \\
170\end{array}$ & $\begin{array}{l}340 \\
341 \\
205\end{array}$ & 2 & $\begin{array}{l}104 \\
212\end{array}$ & 192 & 248 & 229 & 270 & 4.966 & 0.61 \\
\hline 72 & 7 & 14.3 & 0 & 346 & 54 & 120 & $\begin{array}{l}-30 \\
-30\end{array}$ & 170 & $\begin{array}{l}205 \\
210\end{array}$ & 1 & $\begin{array}{l}84 \\
94\end{array}$ & 102 & 92 & 99 & 109 & $\begin{array}{l}0.310 \\
0.441\end{array}$ & 0.77 \\
\hline 12 & 7 & 14.4 & 13 & 6 & 63 & 100 & 10 & 200 & 224 & 1 & 94 & 94 & 106 & 119 & 120 & $\begin{array}{l}0.435 \\
0.649\end{array}$ & \\
\hline $\begin{array}{l}73 \\
74\end{array}$ & $\begin{array}{l}1 \\
1\end{array}$ & $\begin{array}{l}14.5 \\
14.6\end{array}$ & $\begin{array}{r}-10 \\
37\end{array}$ & $\begin{array}{r}340 \\
63\end{array}$ & $\begin{array}{l}44 \\
65\end{array}$ & $\begin{array}{r}270 \\
40\end{array}$ & $\begin{array}{r}-100 \\
80\end{array}$ & $\begin{array}{l}280 \\
190\end{array}$ & $\begin{array}{l}402 \\
210\end{array}$ & $\begin{array}{l}2 \\
1\end{array}$ & $\begin{array}{r}148 \\
88\end{array}$ & & 100 & & & $\begin{array}{l}1.697 \\
0.357\end{array}$ & $\begin{array}{l}1.00 \\
1.00\end{array}$ \\
\hline 75 & 7 & 14.7 & 62 & 103 & 50 & -20 & 90 & 110 & 144 & 1 & 108 & & & & & 0.660 & \\
\hline 76 & 1 & & 60 & 104 & 53 & $\begin{array}{r}-20 \\
-100\end{array}$ & 80 & 11 & 137 & 1 & 123 & 123 & 131 & 123 & 138 & 0.986 & 0.78 \\
\hline 77 & 18 & $\begin{array}{l}14.6 \\
15.1\end{array}$ & 42 & 71 & $\begin{array}{l}44 \\
58\end{array}$ & $\begin{array}{r}-100 \\
70\end{array}$ & 200 & $\begin{array}{l}280 \\
340\end{array}$ & 402 & 2 & $\begin{array}{l}108 \\
152\end{array}$ & & & & & $\begin{array}{l}0.660 \\
1.839\end{array}$ & 1.00 \\
\hline 78 & 2 & & $\begin{array}{l}44 \\
46\end{array}$ & $\begin{array}{l}73 \\
76\end{array}$ & 58 & $\begin{array}{l}60 \\
30\end{array}$ & 200 & 330 & 391 & 2 & 173 & 181 & 175 & 161 & 186 & 2.696 & 0.82 \\
\hline & & 15.3 & 44 & 72 & $\begin{array}{l}20 \\
55\end{array}$ & $\begin{array}{l}30 \\
40\end{array}$ & $\begin{array}{l}120 \\
120\end{array}$ & $\begin{array}{l}18 \\
18\end{array}$ & $\begin{aligned} 218 \\
220\end{aligned}$ & $\begin{array}{l}1 \\
1\end{array}$ & $\begin{array}{l}74 \\
78\end{array}$ & 84 & 74 & 74 & 84 & $\begin{array}{l}0.212 \\
0.248\end{array}$ & 0.88 \\
\hline $\begin{array}{l}79 \\
80\end{array}$ & 1 & $\begin{array}{l}15.3 \\
15.4\end{array}$ & $\begin{array}{l}25 \\
59\end{array}$ & $\begin{array}{l}37 \\
95\end{array}$ & 57 & 120 & $\begin{array}{r}90 \\
250\end{array}$ & $\begin{array}{l}230 \\
280\end{array}$ & $\begin{array}{r}275 \\
376 \\
376\end{array}$ & 2 & $\begin{array}{r}80 \\
\end{array}$ & & & & & 0.268 & 1.00 \\
\hline 81 & 10 & $\begin{array}{l}15.4 \\
15.7\end{array}$ & $\begin{array}{l}59 \\
57\end{array}$ & $\begin{array}{l}95 \\
90\end{array}$ & $\begin{array}{l}48 \\
47\end{array}$ & $\begin{array}{r}-20 \\
0\end{array}$ & $\begin{array}{l}250 \\
180\end{array}$ & $\begin{array}{l}280 \\
190\end{array}$ & $\begin{array}{l}360 \\
262\end{array}$ & 1 & $\begin{array}{l}122 \\
112\end{array}$ & & & & & 0.736 & 1.00 \\
\hline 82 & 3 & 15.8 & 34 & 54 & 50 & 150 & 210 & 310 & 403 & 2 & $\begin{array}{l}135 \\
122\end{array}$ & 146 & 147 & 118 & 163 & $\begin{array}{l}1.292 \\
0.951\end{array}$ & 0.69 \\
\hline 83 & 2 & 15.9 & 73 & 108 & 38 & -60 & 180 & 150 & 242 & 2 & $\begin{array}{r}127 \\
86\end{array}$ & 130 & 128 & 128 & 133 & $\begin{array}{l}1.080 \\
0.333\end{array}$ & 0.92 \\
\hline 84 & 7 & 10.5 & & 100 & 50 & -00 & 100 & 100 & $2 * 2$ & 2 & 90 & 86 & 95 & 86 & 95 & $\begin{array}{l}0.382 \\
0.32\end{array}$ & 0.91 \\
\hline & r & $\begin{array}{l}16.0 \\
16.1\end{array}$ & $\begin{array}{l}40 \\
38\end{array}$ & $\begin{array}{l}63 \\
61\end{array}$ & $\begin{array}{l}48 \\
48\end{array}$ & $\begin{array}{l}100 \\
110\end{array}$ & $\begin{array}{l}200 \\
200\end{array}$ & $\begin{array}{l}250 \\
250\end{array}$ & & $\begin{array}{l}2 \\
2\end{array}$ & $\begin{array}{l}136 \\
149\end{array}$ & 165 & 141 & 143 & 166 & $\begin{array}{l}1.3 \\
1.7\end{array}$ & 0.82 \\
\hline 85 & 3 & 16.1 & -1 & 11 & 34 & 310 & 60 & 210 & 37 & 2 & $\begin{array}{l}172 \\
178\end{array}$ & 179 & 172 & 180 & 181 & $\frac{2}{2}$. & 0.95 \\
\hline 86 & 6 & $\begin{array}{l}16.1 \\
16.2\end{array}$ & $\begin{array}{l}40 \\
40\end{array}$ & $\begin{array}{l}63 \\
65\end{array}$ & $\begin{array}{l}47 \\
46\end{array}$ & $\begin{array}{l}100 \\
100\end{array}$ & $\begin{array}{l}200 \\
210\end{array}$ & $\begin{array}{l}240 \\
240\end{array}$ & $\begin{array}{r}328 \\
334\end{array}$ & $\begin{array}{l}2 \\
2\end{array}$ & $\begin{array}{l}136 \\
146\end{array}$ & 148 & 153 & 136 & 156 & & 0.87 \\
\hline 87 & 4 & 16.3 & 42 & 67 & 45 & 90 & 210 & 230 & 324 & 2 & $\begin{array}{l}132 \\
141\end{array}$ & 138 & 148 & 138 & 149 & $\begin{array}{l}1.204 \\
1.467\end{array}$ & 0.89 \\
\hline 88 & 2 & $\begin{array}{l}16.3 \\
16.3\end{array}$ & $\begin{array}{l}44 \\
42\end{array}$ & $\begin{array}{l}70 \\
67\end{array}$ & 45 & 5 & 140 & 150 & & 1 & $\begin{array}{l}62 \\
70\end{array}$ & 72 & 72 & 72 & 79 & 0.178 & 0.78 \\
\hline 89 & 4 & & 5 & 20 & 35 & 270 & $\begin{array}{l}100 \\
100\end{array}$ & & & 2 & 174 & & & & & & \\
\hline 90 & 1 & & ${ }_{10}^{6}$ & $\begin{array}{l}22 \\
27\end{array}$ & & 27 & 114 & & & & 18 & 184 & 194 & 184 & 196 & & $\begin{array}{l}0.89 \\
1.00\end{array}$ \\
\hline 91 & 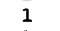 & & 13 & 32 & 3. & 26 & 1 & 20 & & 2 & 174 & & & & & 8 & 1.00 \\
\hline 92 & 17 & & 66 & 90 & 3 & -3 & & & & & & & & & & & \\
\hline 93 & 1 & & 67 & 99 & 7 & -4 & 2 & & & 2 & $\begin{array}{l}171 \\
106\end{array}$ & 170 & 187 & 170 & 196 & & 0.74 \\
\hline 94 & 3 & & 49 & 77 & 35 & 50 & $8+0$ & & 26 & 2 & + & & & & & & \\
\hline 95 & 6 & 17.3 & 52 & $\begin{array}{l}79 \\
94\end{array}$ & 35 & $\begin{array}{r}40 \\
-20\end{array}$ & 280 & 190 & 339 & $\begin{array}{l}2 \\
2\end{array}$ & 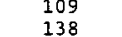 & 110 & 109 & 109 & 116 & $\begin{array}{l}0.672 \\
1.376\end{array}$ & 0.86 \\
\hline & & 11.3 & & 94 & 24 & -20 & 280 & 190 & & & $\begin{array}{l}154 \\
\end{array}$ & 157 & 156 & 148 & 165 & 1.916 & 0.84 \\
\hline
\end{tabular}

distributions (Fig. 8) can be rotated and examined from an arbitrary view point on a computer screen. The crosssections of the 3-D void distribution (Fig. 9) are shown jointly with the distribution of the corresponding tracers in slices of a thickness equal to the grid constant $k=$ $10 h^{-1}$ Mpc.

The 3-D distribution of the voids in Fig. 8 (left panel) suggests a void-filled Universe with closely packed and intersecting voids. However, the cross-sections of the 3-D distribution in Fig. 9 show that the large voids may be separated by large zones of enhanced density of the tracing objects. Such a zone is best outlined in the $y=0 \mathrm{Mpc}$ cross-section of the distribution of the $R \geq 1 \mathrm{~A} / \mathrm{ACO}$ clusters for $z=200-250 h^{-1} \mathrm{Mpc}$ (Fig. 9a), but it can be identified in the other cross-sections, as well as in the adjacent cuts for $y \neq 0$ and $x \neq 0$, although in the direction along the $y$ axis (Fig. 9, right panel) it is smaller and not well outlined. This feature is probably due to the presence of a large orthogonal structure at this distance similar to the Great Wall (Tully 1986, 1987).

\section{Analysis of the void catalogues}

\subsection{Automated comparison of the void catalogues}

The generation of a large number of void catalogues by varying the sample, the search method, or the void selection criterion has evoked the necessity for an easy and objective way to compare the results. AVSAS offers such a possibility, applicable also to the comparison with voids from previous investigations.

The comparison of two void catalogues is based on the comparison of the positions and dimensions of the CS of the voids. The criterion for coincidence of two voids from different catalogues is $\Delta r<\max \left(d_{1}, d_{2}\right)$, where $\Delta r$ is the distance between the centres of the compared CS, and $d_{1}, d_{2}$ are their radii. This criterion must be satisfied for at least one pair of coinciding CS. A rigorous criterion for void coincidence may be chosen by setting $\Delta r=0$. The results from the comparison are output as cross-identification tables with the coinciding voids, and 


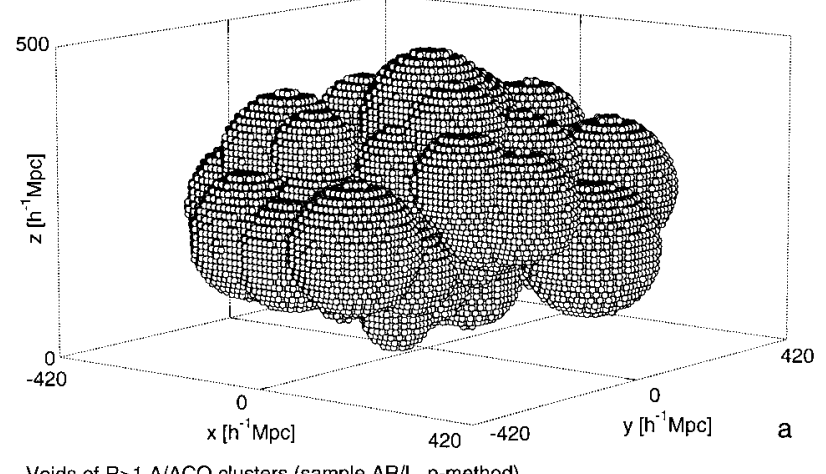

Voids of $R \geq 1$ AVACO clusters (sample $A R / L, p$-method)

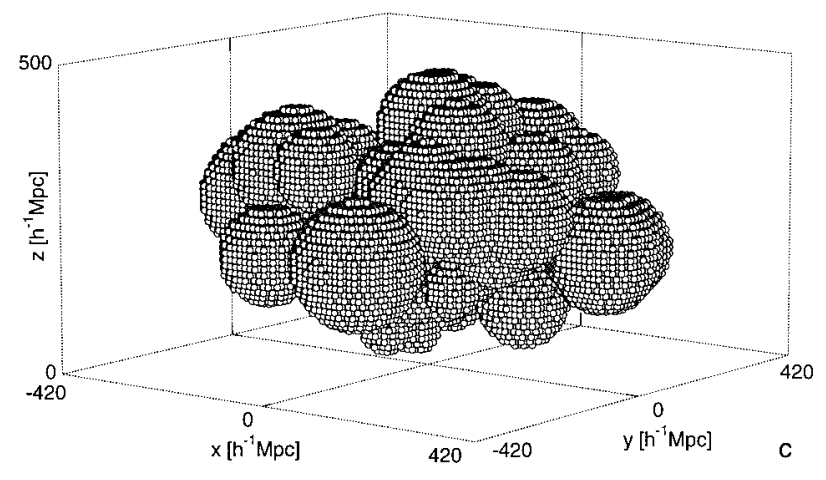

Voids of $R \geq 0$ A/ACO clusters (sample A/L, p-method)
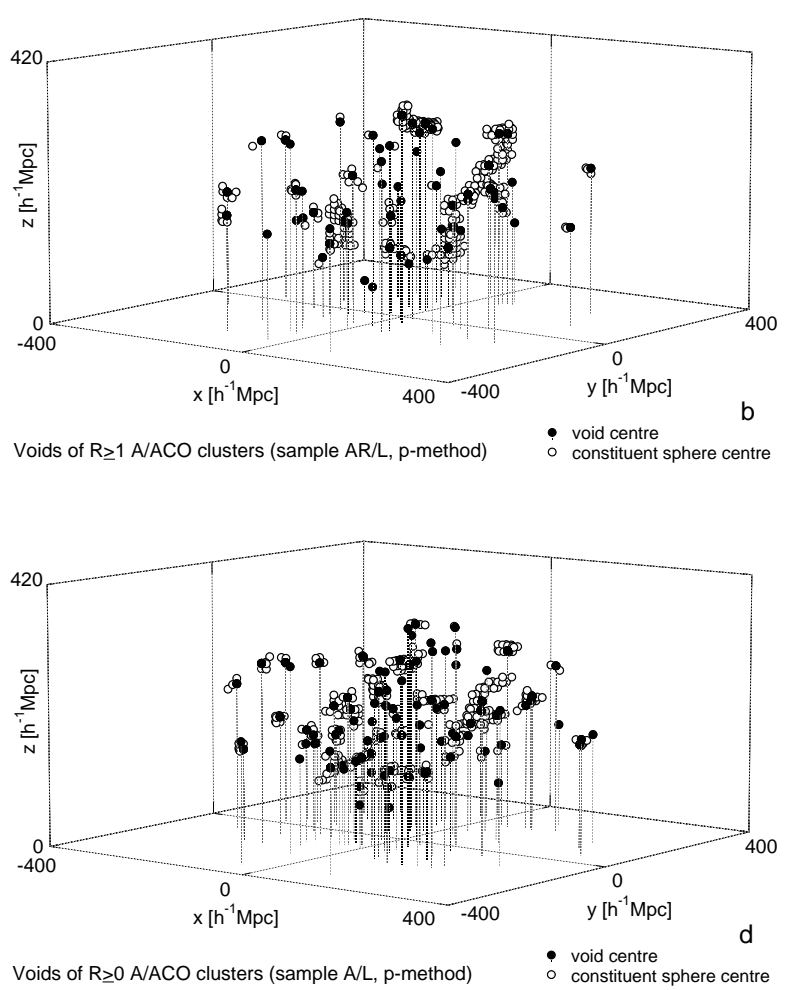

Fig. 8. Spatial distribution of the voids (left panel) and of the void and constituent sphere centres (right panel) from the catalogues in Tables 3 and 4: a and b) voids of $R \geq 1 \mathrm{~A} / \mathrm{ACO}$ clusters (catalogue AR/Lp), $\mathbf{c}$ and $\mathbf{d}$ ) voids of $R \geq 0 \mathrm{~A} / \mathrm{ACO}$ clusters (catalogue A/Lp). Coordinate system and view point are the same as in Fig. 5

as detailed lists of the coinciding CS with the differences in their positions and dimensions.

We have applied both the rigorous and loose criteria to compare the void catalogues listed in Table 2 . Table 5 contains the results of the comparison of the two catalogues $\mathrm{AR} / \mathrm{Lp}$ and A/Lp given in Tables 3 and 4, respectively. The numbers in Table 5 marked by an asterisk correspond to cases satisfying the rigorous criterion $\Delta r=0$. As it is seen in Table 5 most of the voids of $R \geq 1$ clusters (90\%) can be identified with voids of $R \geq 0$ clusters.

We have compared also our void catalogues with voids known from previous investigations. To do this we have composed a compilation of voids in the NGH, hereafter referred to as $\mathrm{VC}$, containing 39 voids of clusters of galaxies from the following sources: (1) Bahcall \& Soneira (1982b) - one void of $R \geq 1$ Abell clusters; (2) Batuski \& Burns (1985) - 22 voids of Abell clusters; (3) Einasto et al. (1994) -16 voids of A/ACO clusters. We shall use the abbreviations $\mathrm{BS}, \mathrm{BB}$, and $\mathrm{E}$ for sources 1-3, respectively.

The compilation VC occupies a volume considerably smaller than the volume occupied by our void catalogues: the void centres reach a limiting distance of about $250 h^{-1}$ $\mathrm{Mpc}$. The dimensions of the voids of clusters from VC correspond roughly to our criterion for void selection $D_{\min }=$ $50 h^{-1} \mathrm{Mpc}$.
Before the comparison, the voids from VC with nonspherical shapes have been reduced to the largest empty spheres which can be nested in them, with the exception of the huge BS void which, because of its extended form, has been approximated with 21 non-overlapping equal spheres with diameters of $60 h^{-1} \mathrm{Mpc}$.

The results of the comparison of the void catalogues $\mathrm{AR} / \mathrm{Lp}$ and A/Lp (Tables 3 and 4 ) with the voids from the compilation VC are given as identification lists in Table 6 , where the first column contains the serial numbers (from Tables 3 and 4) of the voids identified in VC, and the second column contains the identifications from $\mathrm{VC}$, presented with their original numbers from the corresponding literature sources, preceded by the source abbreviation. The comparison shows that about $70 \%$ of the voids of clusters from $\mathrm{VC}$ can be identified in at least one of the two void catalogues. On the other hand, $35-40 \%$ of the voids in these catalogues, most of them in the nearer subvolume $V 1$, are identified in VC. The rest $>60 \%$ of the voids lie predominantly beyond subvolume $V 1$, i.e. in regions not covered by the compilation VC. We can conclude that about $20 \%$ of the voids detected by us in volume $V 1$ and $90 \%$ of the voids outside volume $V 1$ are newly discovered voids or void candidates. The reality of the latter needs to be confirmed by future complete redshift surveys. 

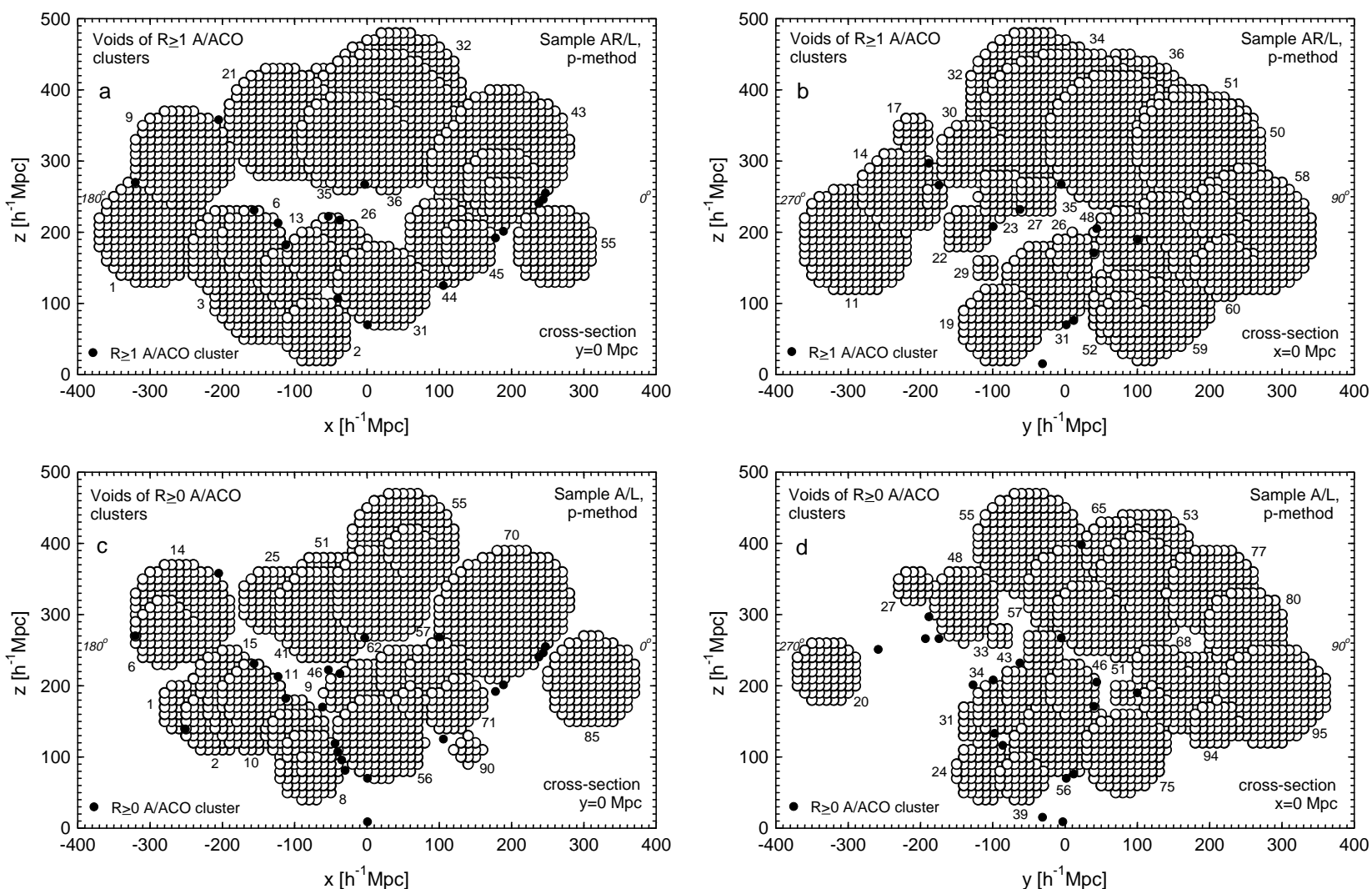

Fig. 9. Central cross-sections in the $x-z$ plane (left panel) and $y-z$ plane (right panel) of the joint spatial distribution of voids and tracers: a-d) same void catalogues as in Fig. 8. Voids are marked with their serial numbers from Tables 3 and 4
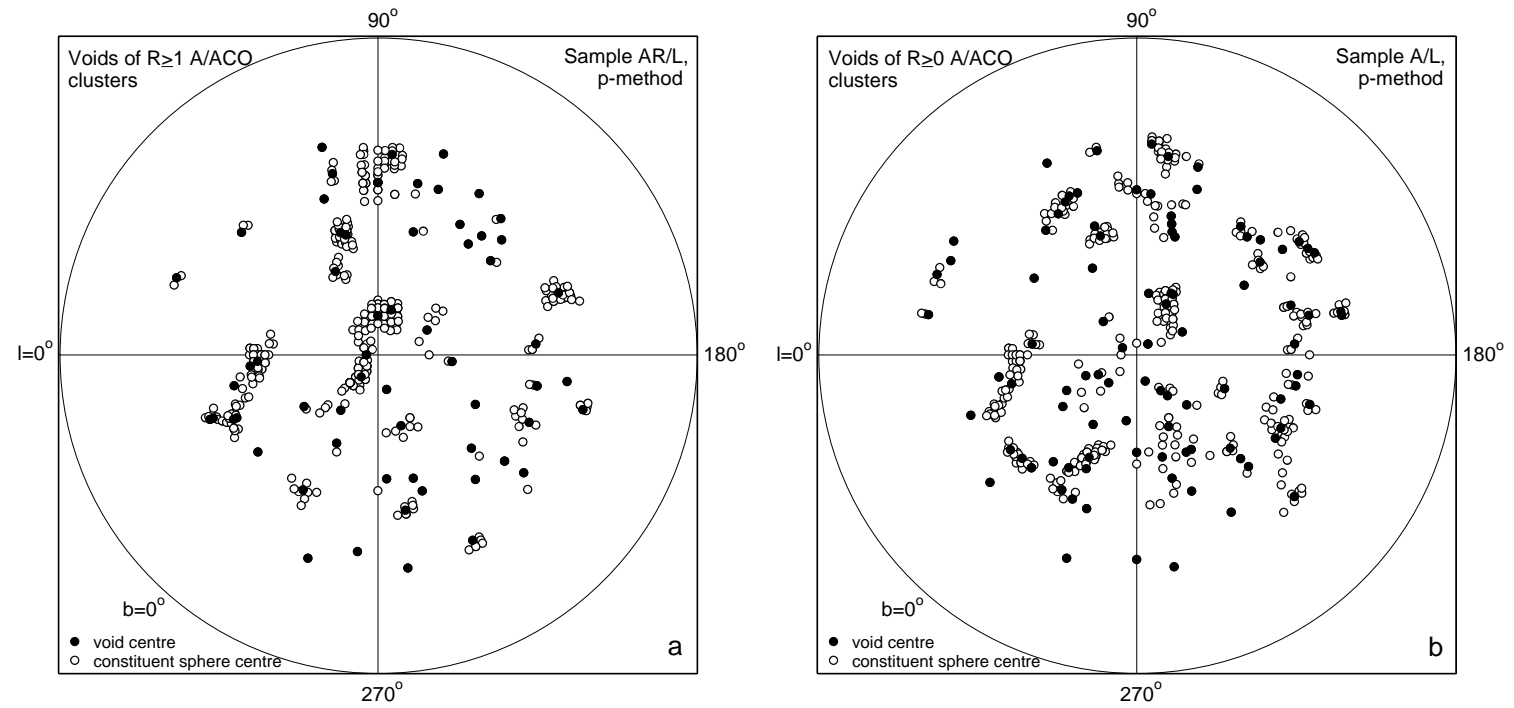

Fig. 10. Surface distribution of the void and constituent sphere centres in a Lambert equal-area projection centred on the NGP: a-b) same void catalogues as in Fig. 8 
Table 5. Identification of the voids of $R \geq 1 \mathrm{~A} / \mathrm{ACO}$ clusters (catalogue $\mathrm{AR} / \mathrm{Lp}$ ) with voids of $R \geq 0$ clusters (catalogue $\mathrm{A} / \mathrm{Lp})$

\begin{tabular}{|c|c|c|c|c|c|}
\hline$A R / L p$ & $\mathrm{~A} / \mathrm{Lp}$ & AR/Lp & $\mathrm{A} / \mathrm{I}$ & & \\
\hline 1 & 6 & 31 & $56^{*}$ & & \\
\hline 2 & 8 & 32 & $55^{*}$ & 57 & \\
\hline 3 & $10 \quad 15$ & 33 & & & \\
\hline 4 & $9 *$ & 34 & 55 & 57 & 60 \\
\hline 5 & $4 \quad 20$ & 35 & 53 & & \\
\hline 6 & $11^{*}$ & 36 & 53 & 60 & 65 \\
\hline 7 & 9* & 37 & 75 & & \\
\hline 8 & 19 & 38 & $61^{*}$ & & \\
\hline 9 & $14^{*}$ & 39 & 59 & & \\
\hline 10 & 17 & 40 & $68^{*}$ & 81 & \\
\hline 11 & 20 & 41 & $70^{*}$ & & \\
\hline 12 & $21^{*}$ & 42 & $70^{*}$ & & \\
\hline 13 & 9 & 43 & $70^{*}$ & & \\
\hline 14 & & 44 & $71^{*}$ & $72 *$ & \\
\hline 15 & & 45 & 71 & & \\
\hline 16 & $26^{*}$ & 46 & $73^{*}$ & & \\
\hline 17 & $27^{*}$ & 47 & 73 & & \\
\hline 18 & 38 & 48 & $74^{*}$ & & \\
\hline 19 & 24 & 49 & $76^{*}$ & & \\
\hline 20 & 23 & 50 & 77 & & \\
\hline 21 & 25 & 51 & $77 *$ & & \\
\hline 22 & & 52 & 75 & & \\
\hline 23 & $33^{*}$ & 53 & 87 & & \\
\hline 24 & $35^{*}$ & 54 & 91 & & \\
\hline 25 & 37 & 55 & 89 & & \\
\hline 26 & 46 & 56 & 86 & 87 & 94 \\
\hline 27 & 44 & 57 & 83 & & \\
\hline 28 & & 58 & $92 *$ & 95 & \\
\hline 29 & $47^{*}$ & 59 & 75 & 88 & \\
\hline 30 & $48^{*}$ & 60 & 94 & & \\
\hline
\end{tabular}

\subsection{Kolmogorov-Smirnov test}

We have applied the two-sample Kolmogorov-Smirnov test in an attempt to estimate the statistical significance of the large voids in the distribution of clusters of galaxies. A similar approach for the voids of galaxies has been used by Ryden \& Turner (1984).

Since the statistic we use for comparison of the observed distribution of clusters with random distributions - the values of the $d$-field - is very sensitive to the sample completeness, we have restricted the test to appropriate subsamples of the samples of $R \geq 1 \mathrm{~A} / \mathrm{ACO}$ clusters.

The observed sample used in the test is a subsample from $\mathrm{AR} / \mathrm{L}$ limited to volume $V 1\left(b \geq+40^{\circ}, z \leq 0.09\right)$ with 128 clusters. It is compared to a random sample generated with the same number of objects in the same volume. The number of points (grid nodes) of the $d$-field in volume $V 1$ is 14736 .

For each run of the test the maximum value of the test statistic $D$ has been compared with the critical value $D_{\alpha}$ for three values of the significance level $\alpha: 0.1,0.05$, and 0.01 . The result for a series of 50 generations of random samples is: the null hypothesis (observed sample and random sample are from the same population) is rejected for all significance levels.
Table 6. Identification of the voids of $R \geq 1$ and $R \geq 0$ A/ACO clusters (catalogues $\mathrm{AR} / \mathrm{Lp}$ and $\mathrm{A} / \mathrm{Lp}$ ) with voids known from previous studies

\begin{tabular}{|c|c|c|c|c|c|c|}
\hline \multirow{2}{*}{$\begin{array}{c}\begin{array}{c}\text { Void } \\
\text { Catalog }\end{array} \\
\text { AR/Lp }\end{array}$} & \multicolumn{3}{|c|}{ Identifications } & \multirow{2}{*}{$\begin{array}{c}\text { Void } \\
\text { Catalog }\end{array}$} & \multicolumn{2}{|c|}{ Identifications } \\
\hline & & & & & E10 & BB09 \\
\hline 2 & BB08 & & & 11 & E12 & \\
\hline 3 & E10 & BB09 & & 13 & BB11 & \\
\hline 4 & E11 & & & 15 & E10 & \\
\hline 6 & E12 & & & 16 & BB10 & \\
\hline 7 & E11 & E12 & BB11 & 18 & BS & \\
\hline 8 & BS & & & 22 & E14 & \\
\hline 13 & E16 & & & 29 & E14 & BB13 \\
\hline 26 & BB16 & & & 31 & E14 & BB 13 \\
\hline 28 & BB21 & & & 32 & E16 & \\
\hline 29 & E17 & & & 34 & BB13 & \\
\hline 31 & E18 & E19 & & 40 & BB16 & \\
\hline 37 & E21 & & & 42 & E17 & \\
\hline 40 & E22 & & & 46 & BB 16 & \\
\hline 44 & BB20 & E20 & & 47 & E17 & \\
\hline 45 & BB20 & & & 56 & E18 & E19 \\
\hline 48 & E23 & BB22 & & 62 & BB19 & \\
\hline 52 & E21 & & & 63 & BB20 & \\
\hline 53 & BB24 & & & 64 & BB20 & \\
\hline 56 & BB24 & & & 68 & E22 & \\
\hline 57 & BB21 & & & 71 & $\mathrm{BB} 20$ & \\
\hline 59 & $\mathrm{E} 21$ & BB26 & & 72 & E20 & \\
\hline \multirow[t]{2}{*}{60} & BB24 & & & 74 & E23 & \\
\hline & & & & 75 & E21 & \\
\hline $\mathbf{A} / \mathbf{L p}$ & & & & 78 & BB22 & \\
\hline 1 & E10 & & & 81 & E22 & \\
\hline 2 & E10 & & & 83 & BB21 & \\
\hline 3 & BS & & & 88 & BB24 & \\
\hline 8 & BS & & & 90 & BB23 & \\
\hline 9 & E11 & E12 & BB11 & & & \\
\hline
\end{tabular}

The relative frequency distributions of the values of the $d$-field (the nearest-neighbour distances) of the observed and random samples for one run of the Kolmogorov-Smirnov test are compared in Fig. 11. The excess of large voids in the observed distribution in comparison with the random distribution is well manifested in this figure.

One could argue that the definite result of the Kolmogorov-Smirnov test is due to the incompleteness of the observed sample. Therefore, we have repeated the test with a more "clean" subsample limited by $b \geq$ $+50^{\circ}$ and $0.0133 \leq z \leq 0.08$, with 76 clusters of galaxies and 6712 grid nodes. As a result from 50 generations of random samples the null hypothesis has been rejected in this case, too.

We conclude that if our samples are not seriously affected by incompleteness, the observed voids in the distribution of $R \geq 1 \mathrm{~A} / \mathrm{ACO}$ clusters are not likely to be random fluctuations. This conclusion is in disagreement with the results of Otto et al. (1986) who have suggested a mechanism of void formation based on the interpretation of rich clusters as rare events in the primordial Gaussian density fluctuations. Our result could possibly indicate that the distribution of rich clusters might be described by a statistic more complicated than a simple Gaussian, as suggested by Plionis et al. (1992). 


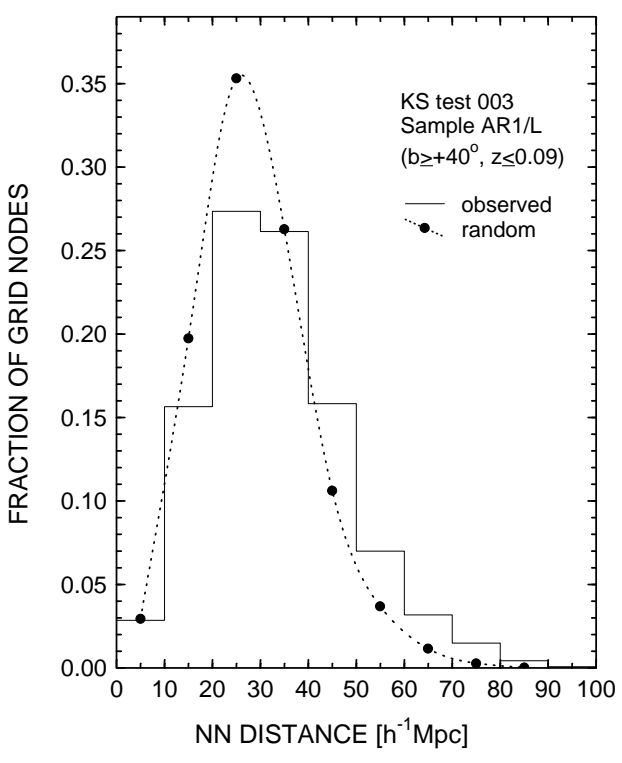

Fig. 11. Comparison of the relative frequency distributions of the distance field values for the observed sample and a random sample generated in one run of the Kolmogorov-Smirnov test. A spline fit is used for the random distribution

\subsection{Statistical analysis of the void catalogues}

The generated large number of void catalogues (Sect. 4) allows for various statistical investigations of the voids, and first of all, for the determination of the mean void characteristics describing the void dimension, volume, and shape.

We have first calculated for all the 16 catalogues generated with the medium-compact grouping option (see Table 2) the mean and median values for the diameters of the largest CS and the equivalent void diameters, the volumes of the largest CS and the total void volumes, as well as the void sphericities, separately for volumes $V 1$ and $V 2$.

The difference between the mean dimensions for volumes $V 1$ and $V 2$ is very large - about $30-35 \%$ for all types of tracers with measured redshifts -, due mainly to the incompleteness in volume $V 2$, and probably partly to the real presence of larger voids in this much larger volume. This difference reduces to $10-15 \%$ when A/ACO clusters with estimated redshifts are used in addition to the clusters with measured redshifts (catalogues Nos. 2, 4, $6,8$, and $10,12,14,16)$. It can be explained as a combined effect of large errors in the estimated cluster distances and the real presence of larger voids in $V 2$ compared with $V 1$.

The comparison of the mean dimensions for different search methods ( $p$ or $\nu$ ) shows that the $\nu$-method gives systematically higher values. Contrary to this, the voids based on different sources of redshift data ( $\mathrm{L}$ or $\mathrm{N}$ ) do not show significant systematic differences in their mean dimensions.

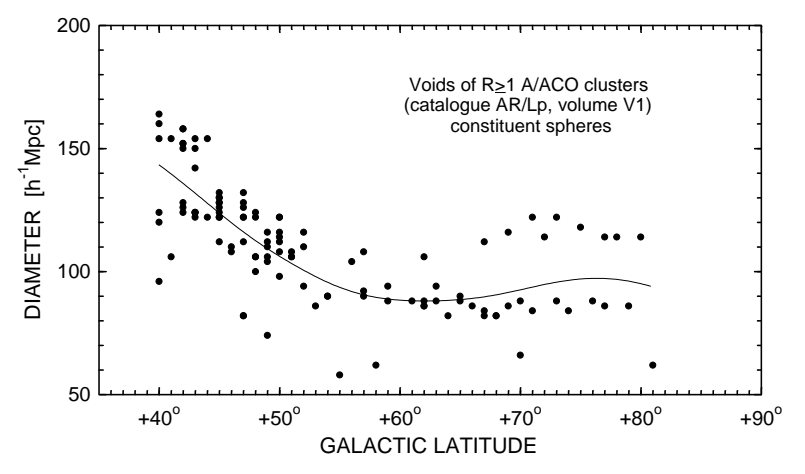

Fig. 12. Diameter of constituent sphere versus galactic latitude for the voids of $R \geq 1 \mathrm{~A} / \mathrm{ACO}$ clusters (catalogue AR/Lp) in volume $V 1$. The line is a least squares fit

To study better the dependence of the mean void dimensions on the completeness of the samples of objects used we have constructed plots of the type diameter distance and diameter - galactic latitude for all void catalogues using the diameters and positions of the centres of the constituent void spheres. Such a plot for catalogue AR/Lp concerning the voids of $R \geq 1 \mathrm{~A} / \mathrm{ACO}$ clusters in volume $V 1$ is presented in Fig. 12 which shows a well defined dependence of the diameters of the CS in volume $V 1$ on the galactic latitude as an apparent presence of larger voids in the latitude zone $+40^{\circ}-+50^{\circ}$. This effect is most probably due to observational selection caused by the galactic obscuration. A similar dependence on the galactic latitude has been found for the other void catalogues, too. Therefore, we conclude that better estimates of the mean void characteristics will be obtained for a subvolume $V 1 \mathrm{~A}$ of volume $V 1$ with $b \geq+50^{\circ}$, in spite of the substantial decrease of the number of voids used for statistics.

A similar check of the dependence of the CS diameters on the distance has shown that the dimensions of the voids in volume $V 1$ are not significantly affected by the observational selection due to the distance.

As seen from Table 2 in Sect. 4, we have generated four void catalogues for each of the four tracer types (AR, $\mathrm{ARE}, \mathrm{A}, \mathrm{AE})$, corresponding to the four combinations of redshift data source and search method: $\mathrm{Lp}, \mathrm{L} \nu, \mathrm{Np}, \mathrm{N} \nu$. Since we do not give priority to any of these combinations, we use all of them to obtain estimates of the mean void characteristics, assuming the corresponding four void catalogues to be independent realizations of the void distribution. Such an averaging over the source of data and search method increases the number of voids used for statistics, decreasing in this way the statistical errors.

The results of the estimation of the mean void characteristics - dimension, volume and shape (sphericity) - are presented in Table 7 with the following columns: Col. (1) - serial numbers of the void catalogues used for the calculation of the mean characteristics (see Table 2); Col. (2) 
- tracer type (see Table 2); Col. (3) - sample volume: $V 1 \mathrm{~A}$ defined by $b \geq+50^{\circ}$ and $z \leq 0.09, V 2 \mathrm{~A}$ defined by $b \geq+50^{\circ}$ and $z \leq 0.14$; Col. (4) - number of voids (sum of the number of voids in the catalogues listed in Col. (1)); Col. (5) - dimension type: $D$ is the diameter of the largest constituent sphere of the void, $D_{\mathrm{e}}$ is the equivalent void diameter (see Sect. 3.2); Col. (6) - mean value of the dimension; Col. (7) - error of the mean for significance level $\alpha=0.05$ (95\% confidence interval): $\epsilon=t_{\alpha_{n}} \sigma_{n} / \sqrt{n}$, where $t_{\alpha_{n}}$ is Student's distribution, $\sigma_{n}$ is the standard deviation, and $n$ is the number of voids; Col. (8) - standard deviation; Col. (9) - median; Col. (10) - volume type: $V$ is the volume of the largest CS of the void, $V_{\mathrm{T}}$ is the total void volume; Cols. (11)-(14) - same as Cols. (6)-(9), but for the void volume; Cols. (15)-(18) - same as Cols. (7)-(9), but for the void sphericity.

Histograms of the void diameters $\left(D\right.$ and $\left.D_{\mathrm{e}}\right)$ for the four samples of voids used to calculate the mean void characteristics (see Table 7) are given in Fig. 13.

We consider as most representative the estimates of the mean void characteristics in Table 7 for the voids of $R \geq 1 \mathrm{~A} / \mathrm{ACO}$ clusters in sample volume $V 1 \mathrm{~A}$, since they are based on most complete samples of the tracing objects. The other estimates in Table 7 are affected to a larger extent by sample incompleteness, and/or by large errors of the estimated redshifts. It is seen that for tracer types ARE and AE (i.e. samples of clusters with measured or estimated redshifts) the estimated dimensions in volume $V 2 \mathrm{~A}$ are $10-15 \%$ higher than in $V 1 \mathrm{~A}$.

We consider the equivalent diameter $D_{\mathrm{e}}$ as a more representative estimate of the void dimensions than $D$, since it takes into account the total void volume. Thus, the best estimate for the mean dimension of the voids of $R \geq 1 \mathrm{~A} / \mathrm{ACO}$ clusters is $D_{\mathrm{e}}=105.0 \pm 5.6 h^{-1} \mathrm{Mpc}$, and for the voids of $R \geq 0 \mathrm{~A} / \mathrm{ACO}$ clusters we have $D_{\mathrm{e}}=87.2 \pm 4.1 h^{-1} \mathrm{Mpc}$ (see Table 7 ). The corresponding total void volumes are $V_{\mathrm{T}}=0.667 \pm 0.10210^{6} h^{-3} \mathrm{Mpc}^{3}$ and $V_{\mathrm{T}}=0.400 \pm 0.06010^{6} h^{-3} \mathrm{Mpc}^{3}$.

It is seen from the last two columns of Table 7 that the voids have quite spherical shapes independent of the tracer type. This result is in agreement with the qualitative estimate of the shapes of the voids of clusters as "remarkably spherical" by Batuski \& Burns (1985). The mean sphericity for the voids of $R \geq 1 \mathrm{~A} / \mathrm{ACO}$ clusters is $0.88 \pm 0.03$, and for the voids of $R \geq 0 \mathrm{~A} / \mathrm{ACO}$ clusters it is $0.91 \pm 0.02$, i.e. the deviation from an ideal sphere is about $10 \%$. The estimates of the mean sphericity are influenced to a certain extent by the choice in the search procedure of a comparatively high value $D_{\min }=50 \mathrm{~h}^{-1}$ $\mathrm{Mpc}$ for the minimum void dimension.

Our results for the mean dimensions of the voids of clusters are in good agreement with those of Einasto et al. (1997). Using a sample of $1304 \mathrm{~A} / \mathrm{ACO}$ clusters in both galactic hemispheres to a limiting distance $z=0.12,1 / 3$ of which with estimated distances, they have obtained for the median diameters of the largest empty spheres estimates of $90 h^{-1} \mathrm{Mpc}$ for the voids of $R \geq 0 \mathrm{~A} / \mathrm{ACO}$ clusters, $100 h^{-1} \mathrm{Mpc}$ for the voids of clusters belonging to superclusters, and $110 h^{-1} \mathrm{Mpc}$ for the voids of clusters belonging to rich superclusters. Taking into consideration the tracer type, the sample volume, and the type of dimension, most appropriate for the comparison with their results are our estimates for the median diameters of the largest CS (dimension type $D$ ) for tracer types $\mathrm{AE}$ and ARE in sample volume $V 2 \mathrm{~A}$. (Note, however, the difference between our tracer type ARE and the corresponding tracer types in Einasto et al. (1997) based on the membership of clusters in superclusters.) We have for these cases in Table 7 median diameters, respectively, $92.0 h^{-1}$ Mpc against their value $90 h^{-1} \mathrm{Mpc}$, and $106.0 h^{-1} \mathrm{Mpc}$ against their values 100 and $110 h^{-1}$ Mpc. However, if the comparison is made with our best estimates, which, as already explained, correspond to volume $V 1 \mathrm{~A}$, and not to $V 2$ A, i.e. with median diameters $80.0 h^{-1} \mathrm{Mpc}$ for tracer type A, and $94.0 h^{-1} \mathrm{Mpc}$ for tracer type AR (see Table 7) then we see that our estimates are lower by $5-10 \%$. We suppose that one reason for the larger median diameters of Einasto et al. (1997) may be an unremoved effect of the galactic obscuration due to the use of voids situated at low galactic latitudes.

It is also interesting to compare our results with theoretical predictions for the size of voids of clusters. Frisch et al. (1995) have obtained for the mean diameters of the largest empty spheres of the voids of rich clusters $(R \geq 0)$ for three different models the following estimates: $91.3 \pm 11.6 h^{-1} \mathrm{Mpc}$ for a two-power law model, $82.6 \pm$ $12.5 h^{-1}$ Mpc for a CDM model with density parameter $\Omega=1$, and $90.5 \pm 14.4 h^{-1} \mathrm{Mpc}$ for a low-density CDM model with $\Omega=0.2$. These values have been calculated with the Bahcall \& Cen (1993) estimate $\bar{n}=$ $13.510^{-6} h^{3} \mathrm{Mpc}^{-3}$ for the mean number density of the $R \geq 0$ clusters with which our samples of $R \geq 0$ clusters are in good agreement (see Sect. 2.1). Our estimate $82.3 \pm 3.1 h^{-1} \mathrm{Mpc}$ in Table 7 (for tracer type A, sample volume $V 1 \mathrm{~A}$, and dimension type $D$ ) is in best agreement with the high-density CDM model with $\Omega=1$. However, the uncertainties of the model estimates are too large in order to make a definite discrimination between the three models.

\section{Void population and void shell}

By definition the voids identified in the present study are regions completely devoid of a certain type of objects. They, however, may be populated by other types of objects of lower richness class.

Studies of the voids in the distribution of galaxies like the large void in Boötes with a population of $\sim 60$ galaxies discovered so far (Szomoru et al. 1996), do not confirm the theoretical predictions for the presence of a significant population of dwarf galaxies or of giant, unevolved, low 
Table 7. Mean void characteristics

\begin{tabular}{|c|c|c|c|c|c|c|c|c|c|c|c|c|c|c|c|c|c|}
\hline \multirow[b]{2}{*}{$\begin{array}{c}\text { Void Catalog } \\
\text { No. }\end{array}$} & \multirow[b]{2}{*}{$\begin{array}{c}\text { Tracer } \\
\text { type }\end{array}$} & \multirow[b]{2}{*}{$\begin{array}{l}\text { Sample } \\
\text { volume }\end{array}$} & \multirow[b]{2}{*}{$n$} & \multicolumn{5}{|c|}{ DIMENSION } & \multicolumn{5}{|c|}{ VOLUME } & \multicolumn{4}{|c|}{ SPHERICITY } \\
\hline & & & & \multicolumn{3}{|c|}{$\begin{array}{c}\text { Type MEAN } \pm \epsilon \\
{\left[h^{-1} \mathrm{Mpc}\right]}\end{array}$} & $\sigma$ & $\begin{array}{l}\text { MEDIAN } \\
{\left[h^{-1} \mathrm{Mpc}\right]}\end{array}$ & \multicolumn{3}{|c|}{$\begin{array}{c}\text { Type MEAN } \pm \in \\
{\left[10^{6} h^{-3} \mathrm{Mpc}^{3}\right]}\end{array}$} & \multicolumn{2}{|c|}{$\begin{array}{c}\sigma \quad \text { MEDIAN } \\
\quad\left[10^{6} h^{-3} \mathrm{Mpc}^{3}\right]\end{array}$} & MEAN & \multirow[t]{2}{*}{$\pm \epsilon$} & \multirow[t]{2}{*}{$\sigma$} & MEDIAN \\
\hline \multirow[t]{2}{*}{$1,3,5,7$} & $\mathrm{AR}$ & V1A & 49 & $D$ & 96.8 & 4.4 & 15.4 & 94.0 & $v$ & 0.510 & 0.065 & 0.225 & 0.435 & & & & \\
\hline & & & & $D_{\mathbf{e}}$ & 105.0 & 5.6 & 19.4 & 105.7 & $V_{T}$ & 0.667 & 0.102 & 0.356 & 0.619 & 0.88 & 0.03 & 0.12 & 0.92 \\
\hline \multirow[t]{2}{*}{$2,4,6,8$} & ARE & $\mathrm{V} 2 \mathrm{~A}$ & 191 & $D$ & 109.4 & 3.8 & 26.6 & 106.0 & V & 0.808 & 0.082 & 0.573 & 0.624 & & & & \\
\hline & & & & $D_{\mathrm{e}}$ & 118.7 & 4.7 & 32.9 & 112.6 & $V_{\mathrm{T}}$ & 1.086 & 0.130 & 0.913 & 0.748 & 0.88 & 0.02 & 0.11 & 0.89 \\
\hline \multirow[t]{2}{*}{$9,11,13,15$} & A & V1A & 89 & $D$ & 82.3 & 3.1 & 14.8 & 80.0 & $V$ & 0.321 & 0.036 & 0.172 & 0.268 & & & & \\
\hline & & & & $D_{\mathrm{e}}$ & 87.2 & 4.1 & 19.4 & 83.1 & $V_{\mathrm{T}}$ & 0.400 & 0.060 & 0.284 & 0.301 & 0.91 & 0.02 & 0.12 & 1.00 \\
\hline \multirow[t]{2}{*}{$10,12,14,16$} & $\mathrm{AE}$ & $\mathrm{V} 2 \mathrm{~A}$ & 271 & $D$ & 92.1 & 2.5 & 21.3 & 92.0 & $V$ & 0.477 & 0.040 & 0.334 & 0.408 & & & & \\
\hline & & & & $D_{\mathrm{e}}$ & 99.0 & 3.2 & 27.2 & 95.8 & $V_{T}$ & 0.629 & 0.066 & 0.550 & 0.461 & 0.90 & 0.01 & 0.11 & 0.91 \\
\hline
\end{tabular}
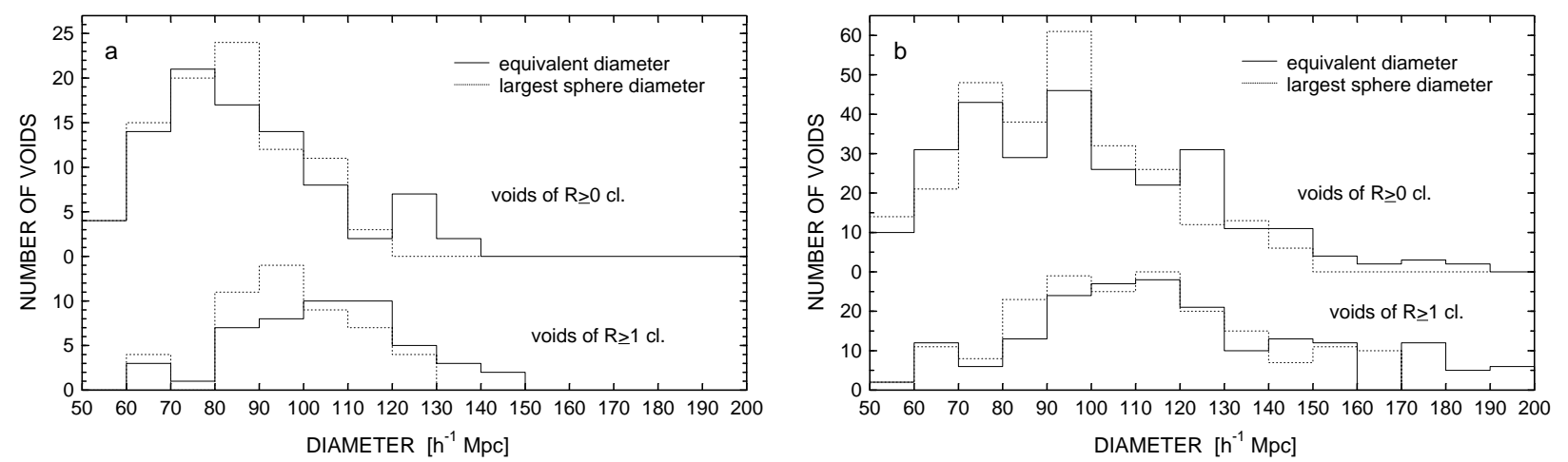

Fig. 13. Number versus diameter (equivalent and of the largest empty sphere) for voids of $R \geq 1$ and $R \geq 0 \mathrm{~A} / \mathrm{ACO}$ clusters a) with measured redshifts (volume $V 1 \mathrm{~A}$ ), and b) with measured or estimated redshifts (volume $V 2 \mathrm{~A}$ )

surface brightness galaxies in the voids. Voids are rather inhabited by galaxies which are similar to field galaxies of the same morphological type. They are not uniformly distributed in the voids but form filament or sheet-like structures which surround smaller voids (see e.g. Szomoru et al. 1996; Weistrop et al. 1995; Cruzen et al. 1997; Sage et al. 1997). This picture is in agreement with the idea of a void hierarchy. The latter has been studied in more detail and on a higher hierarchical level by Lindner et al. (1995, 1996) in the region of the Northern Local Supervoid. In an earlier investigation of the $40 h^{-1}$ Mpc Pisces-Cetus void (Burns et al. 1988) in the distribution of Abell clusters Willick et al. (1990) find that it contains filaments of galaxies which surround subvoids with sizes of $25-30 h^{-1} \mathrm{Mpc}$.

Our study of the population in the voids of galaxy clusters is based on a joint treatment of the catalogues of voids (Sect. 4, Table 2) and samples of objects (clusters, groups, galaxies) extracted from the compilations CL, CN, and CG (see Sect. 2.1), by using AVSAS. AVSAS offers possibilities for: (1) complete identification of the population of a chosen type (sample) in each void of a chosen catalogue, (2) identification of the objects surrounding a void in a shell of chosen thickness, (3) construction of the radial distribution of the void and shell population (void profiles), (4) construction of the spatial distribution of the void and shell population. Let us note that by "shell" we shall understand a zone of a chosen thickness around a void which strictly follows the void shape. Thus, the shell may have a very complicated shape if the void is composed of a large number of CS. The shell has a simple spherical form only in the case of a single-sphere void.

Since voids in our void catalogues may overlap we permit a given object to populate more than one void if it is in the overlapping zone. The same is naturally true for the shell when it divides two neighbouring voids.

Because of the large number of void catalogues and voids a complete study of the population of the individual voids is only possible in a separate investigation. Here we shall adduce only first results concerning the population in the voids of $R \geq 1 \mathrm{~A} / \mathrm{ACO}$ clusters. Let us also note that because of the lower completeness limits of the samples of poorer clusters, groups, and galaxies in comparison with the samples of rich A/ACO clusters, the results in the present section should be considered as preliminary.

The joint processing in the volume defined by $b \geq$ $+20^{\circ}$ and $z \leq 0.16$ of (1) the void catalogue AR/Lp (60 voids, see Table 3), a sample of 1312 clusters and groups extracted from the compilation CL, and a sample of 19879 galaxies extracted from the compilation CG (see Sect. 2.1), and (2) the void catalogue AR/Np (65 voids, 
see Table 2), a sample of 1309 clusters and groups extracted from the compilation $\mathrm{CN}$, and the same sample of galaxies as in (1), has led to the complete identification of the void population of poorer clusters (i.e. $R=0 \mathrm{~A} / \mathrm{ACO}$ and non-A/ACO), groups, and galaxies, as well as of the shell population $(R \geq 1 \mathrm{~A} / \mathrm{ACO}$ clusters, poorer clusters, groups, and galaxies), for each void of the two catalogues. A shell thickness of $10 h^{-1} \mathrm{Mpc}$ has been chosen. This is twice the typical thickness of the walls of the voids of galaxies (e.g., de Lapparent et al. 1991; Doroshkevich et al. 1996). The results from the identification procedure are output as lists of the void and shell populations of the individual voids, and as tables with the numbers of objects of different types in each void and its shell, and the number densities of the void populations. These data for the void catalogue AR/Lp are given in Table 8 where Col. (2) contains the distance from the observer to the centre of the largest void sphere (see Table 3), Cols. (3) and (4) contain the number and density of the void population of poorer clusters and groups, Cols. (5) and (6) contain the number and density of the population of galaxies in the voids, and Cols. (7), (8), and (9) contain the number of $R \geq 1 \mathrm{~A} / \mathrm{ACO}$ clusters forming the void shell, the number of poorer clusters and groups in the shell, and the number of galaxies in the shell, respectively. A similar table has been obtained for void catalogue AR/Np. Table 8 contains only the voids whose centres lie in the near subvolume $V 1$, because of the very high incompleteness outside of it. Even this volume, however, is deeper than the completeness limits of the populations of poorer clusters, groups, and galaxies. Therefore, several of the nearest $\left(r \lesssim 150 h^{-1} \mathrm{Mpc}\right)$ voids show much higher number densities compared to the rest of the voids.

As seen from Table 8 we have divided roughly the void population into two categories: (1) poorer clusters (all types of clusters except the $R \geq 1 \mathrm{~A} / \mathrm{ACO}$ ) and groups, and (2) galaxies. For the shell population we add to these two categories the $R \geq 1 \mathrm{~A} / \mathrm{ACO}$ clusters. Our grounds for not processing the population of groups separately from the poorer clusters are (1) the comparatively small number of groups with measured redshifts at the distances of the voids of rich clusters, and (2) the uncertainty in classifying a poor concentration of galaxies as "poor cluster" or "group" (see e.g. Noonan 1973; Ledlow et al. 1996).

Because of the incompleteness problem the data in Table 8 cannot be used to obtain a reliable estimate of the mean number density of the void population. We may try to get rough estimates based on the nearest voids. From voids Nos. 31, 37, 52 (Table 8) and two additional voids (Nos. 35, 57) from the void catalogue $\mathrm{AR} / \mathrm{Np}$ we obtain the mean number densities of the void population of poorer clusters and groups $\sim 4010^{-6} h^{3} \mathrm{Mpc}^{-3}$, and $\sim 110^{-3} h^{3} \mathrm{Mpc}^{-3}$ for the population of galaxies. (We do not use the nearest void No. 2 in Table 8 because of the exceptionally high number density of its population.) These values are much lower than the known estimates
Table 8. Numbers and densities of the void populations and numbers of the shell populations for the voids of $R \geq 1 \mathrm{~A} / \mathrm{ACO}$ clusters (catalogue AR/Lp, volume $V 1$ )

\begin{tabular}{|c|c|c|c|c|c|c|c|c|}
\hline \multirow[b]{2}{*}{$\begin{array}{l}\text { Void } \\
\text { No. }\end{array}$} & \multirow[b]{2}{*}{$\begin{array}{c}r \\
{\left[h^{-1} \mathrm{Mpc}\right]}\end{array}$} & \multicolumn{4}{|c|}{ VOID } & \multicolumn{3}{|c|}{ SHELL } \\
\hline & & $n_{\mathrm{cg}}$ & $\begin{array}{l}\text { Density } \\
\left.0^{-6} h^{3} \mathrm{Mpc}^{-3}\right]\end{array}$ & $n_{\text {gal }}$ & $\begin{array}{c}\text { Density } \\
{\left[10^{-3} h^{3} \mathrm{Mpc}^{-3}\right]}\end{array}$ & $n_{R \geq 1}$ & $n_{\mathrm{cg}}$ & $n_{\text {gal }}$ \\
\hline 2 & 93 & 69 & 149.7 & 1265 & 2.744 & 7 & 53 & 1046 \\
\hline 3 & 248 & 27 & 6.2 & 363 & 0.083 & 23 & 12 & 291 \\
\hline 4 & 169 & 5 & 6.9 & 104 & 0.144 & 8 & 10 & 244 \\
\hline 6 & 243 & 3 & 3.9 & 38 & 0.049 & 11 & 1 & 62 \\
\hline 7 & 185 & 11 & 9.1 & 290 & 0.240 & 9 & 14 & 172 \\
\hline 8 & 231 & 1 & 2.9 & 18 & 0.053 & 6 & 3 & 49 \\
\hline 10 & 251 & 1 & 4.9 & 10 & 0.049 & 7 & 4 & 31 \\
\hline 13 & 192 & 10 & 16.2 & 100 & 0.162 & 9 & 1 & 159 \\
\hline 22 & 247 & 3 & 19.1 & 14 & 0.089 & 8 & 7 & 23 \\
\hline 26 & 213 & 8 & 10.6 & 197 & 0.261 & 17 & 5 & 242 \\
\hline 27 & 253 & 2 & 16.3 & 6 & 0.049 & 6 & 2 & 50 \\
\hline 28 & 237 & 3 & 8.8 & 13 & 0.038 & 6 & 3 & 46 \\
\hline 29 & 195 & 6 & 5.3 & 217 & 0.192 & 12 & 20 & 251 \\
\hline 31 & 125 & 79 & 62.6 & 2183 & 1.730 & 16 & 57 & 2061 \\
\hline 37 & 124 & 26 & 28.1 & 556 & 0.601 & 5 & 24 & 493 \\
\hline 40 & 264 & 1 & 1.3 & 58 & 0.078 & 10 & 4 & 66 \\
\hline 44 & 197 & 7 & 7.7 & 155 & 0.169 & 17 & 7 & 184 \\
\hline 45 & 230 & 1 & 2.2 & 9 & 0.020 & 9 & 2 & 148 \\
\hline 48 & 232 & 6 & 9.4 & 149 & 0.233 & 24 & 5 & 409 \\
\hline 52 & 129 & 32 & 33.7 & 639 & 0.673 & 5 & 28 & 579 \\
\hline
\end{tabular}

of the mean spatial number density for the two types of population. E.g., Frisch et al. (1995) give for the mean density of the near Zwicky clusters (which are only a part of the total population of poorer clusters and groups in our voids) the value $75.610^{-6} h^{3} \mathrm{Mpc}^{-3}$, and Lin et al. (1996) estimate the mean number density of the galaxies with absolute magnitude $M \leq-17.5+5 \log h$ from the Las Campanas Redshift Survey as $\bar{n}=0.029 h^{3} \mathrm{Mpc}^{-3}$. If our estimates are not strongly affected by the observational selection we may conclude that the voids of $R \geq$ $1 \mathrm{~A} / \mathrm{ACO}$ clusters are also regions with underdensities of the poorer clusters and groups, and of the galaxies.

One of the important questions concerning the void population is how it is distributed inside the void. As a first step we have studied the radial distribution of the number density of the population, i.e. the void density profiles.

Profiles of voids observed in the distribution of galaxies have been obtained so far by Dey et al. (1990), Szomoru et al. (1996), and Lindner et al. (1996). Here, an attempt is made to construct radial profiles of voids in the distribution of clusters of galaxies.

The void radial profiles have been obtained from the population number densities in concentric spheres, centred in the void (on the centroid of all CS), increasing their radius by $\Delta d=5 h^{-1} \mathrm{Mpc}$, i.e. the profiles are cumulative functions. This approach has the disadvantage that it does not take into account the void shape if it is different from spherical. That leads to errors in the calculated densities, which are larger if the void sphericity is smaller. As a result, the zone of the void profile corresponding to the void shell appears artificially extended and flattened. Another 

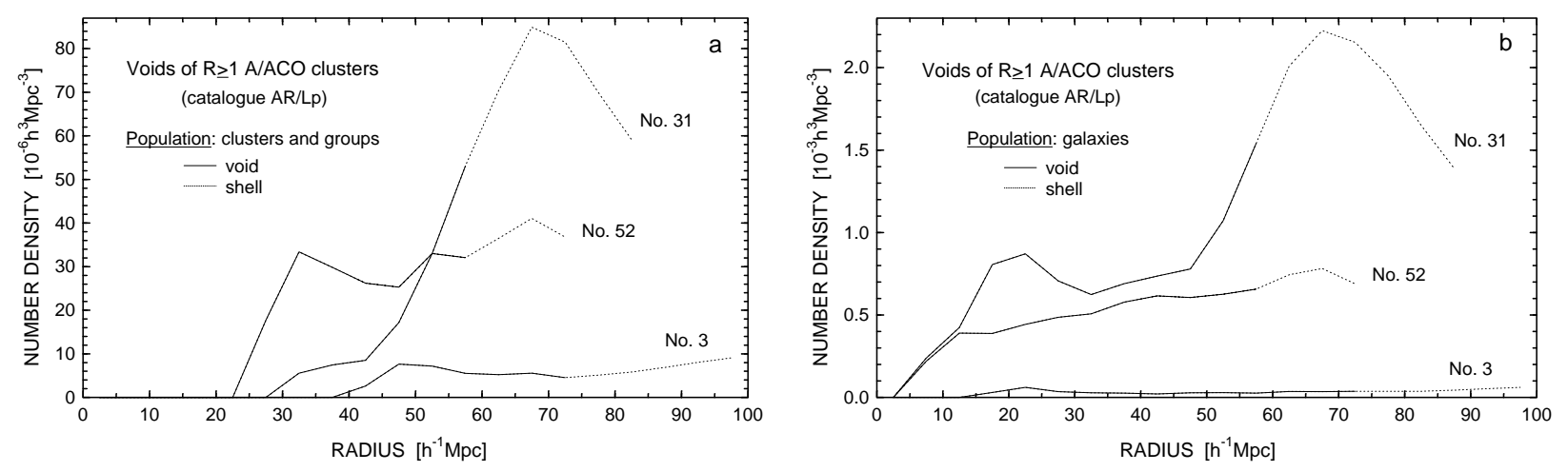

Fig. 14. Radial density profiles of voids of $R \geq 1 \mathrm{~A} / \mathrm{ACO}$ clusters (catalogue AR/Lp, see Table 3) for the population of a) clusters and groups, and $\mathbf{b}$ ) galaxies

disadvantage is that near the void centre the density is averaged over very small volumes.

The radial density profiles of three voids of $R \geq 1$ $\mathrm{A} / \mathrm{ACO}$ clusters selected from the void catalogue AR/Lp (Nos. 3, 31, 52 - see Tables 3 and 8) are shown in Figs. 14a and $b$ for the population of clusters and groups, and of galaxies, respectively. It is seen that the profiles of the more distant void No. 3 are shallower than those of the nearer voids Nos. 31 and 52, probably due to the growing incompleteness with distance. Let us note that the inadequate completeness of the population in most of the voids in volume $V 1$ makes difficult the construction of reliable mean radial profiles from the profiles of the individual voids.

The profile of void No. 31 for the population of clusters and groups shows a deep void with a dense shell. Its population of 79 poorer clusters and groups is concentrated near the void shell, which is formed by $16 R \geq 1 \mathrm{~A} / \mathrm{ACO}$ clusters and contains additionally 57 poorer clusters and groups. The maximum density of the shell is at radius $65-70 h^{-1} \mathrm{Mpc}$ and corresponds to the equivalent diameter $D_{\mathrm{e}}=134 h^{-1} \mathrm{Mpc}$ of the void (see Table 3 ). The central part of the void with diameter $\sim 60 h^{-1} \mathrm{Mpc}$ is completely empty of poorer clusters and groups. However, from the profile of the same void for the population of galaxies (Fig. 14b) it is seen that this central part contains a population of galaxies, penetrating deep into the void. The local maximum of the profile at $\sim 20 h^{-1} \mathrm{Mpc}$ is an indication for the presence of a fine substructure in the void (concentrations and filaments of galaxies) and probably represents the shell of a subvoid of galaxies. This, however, should be confirmed by investigating the 3-D distribution of the void population.

Void No. 52 shows a somewhat different profile from void No. 31. It is shelf-like with the density of the void shell (composed of $5 R \geq 1 \mathrm{~A} / \mathrm{ACO}$ clusters, 28 poorer clusters and groups, 579 galaxies) being not much different from the density of the void population (32 poorer clusters and groups, 639 galaxies). The population of poorer clusters and groups penetrates deep into the void but leaves com- pletely empty a subvoid of nearly $50 h^{-1}$ Mpc (Fig. 14a), which as seen from Fig. 14b is populated by galaxies almost to the void centre.

The density profiles in Figs. 14a and b, as well as density profiles of some other voids that we have studied, suggest that the voids of $R \geq 1 \mathrm{~A} / \mathrm{ACO}$ clusters contain smaller voids of poorer clusters and groups which contain still smaller voids or underdense regions in the distribution of galaxies. This suggestion supports the model of a void hierarchy.

A further, more complete study of the void substructure is possible by direct analysis of the $3-\mathrm{D}$ distribution of the void population. We intend to consider this problem in a separate paper.

\section{Summary}

We have presented in this paper the so far most complete mapping of the large $\left(\geq 50 h^{-1} \mathrm{Mpc}\right)$ voids of clusters of galaxies in the Northern Galactic Hemisphere for $b \geq$ $+30^{\circ}$ to a limiting distance of $420 h^{-1} \mathrm{Mpc}$. Abell/ACO clusters with spectroscopically measured redshifts, as well as with estimated redshifts, have been used as tracers of the large-scale structure, combining the data from four different redshift sources. The analysis of the spatial number density has shown that the $R \geq 1 \mathrm{~A} / \mathrm{ACO}$ clusters can form complete samples to a limiting distance of $\sim 270 h^{-1}$ Mpc, while the samples of $R \geq 0$ clusters are incomplete even in this nearer distance range.

Eight observational samples have been composed and processed for two types of tracers: (1) $R \geq 1 \mathrm{~A} / \mathrm{ACO}$ clusters, and (2) $R \geq 0 \mathrm{~A} / \mathrm{ACO}$ clusters, varying for each tracer type the priority of the source of redshift data (LEB or NED), and varying, as well, the redshift type (measured redshifts versus measured or estimated redshifts).

The observational samples have been processed with the help of an Automated Void Search and Analysis System (AVSAS). The void-search algorithm in AVSAS is based on an identification of the local maxima in 
the nearest-neighbour distance field, defined on a regular grid. Two alternative methods, the $p$-method and the $\nu$-method, are used to identify the local maxima. The first one searches the maxima by direct comparisons of the distance field values, and the second one uses for this purpose the standard deviation of the field values. The number of detected local maximum points depends on the choice of an appropriate value for a parameter $p$ for the first method, respectively $\nu$ for the second method. Next, the search algorithm groups the local maximum points according to their mutual positions on the basis of a choice between three possible options for grouping compact, medium-compact, and loose. Each group of local maximum points identifies a separate void, which is approximated by a system of overlapping empty spheres. The approximation of a void by more than one sphere (the largest empty sphere) allows for a more complete description of the void properties - dimension, volume and shape.

The tests of the void-search procedure have shown that it can be optimized by choosing such values of the parameters $p$ and $\nu$ which maximize the number of detected voids for a minimum degree of overlap of the neighbouring voids.

Choosing a grid constant $k=10 h^{-1} \mathrm{Mpc}$ and a minimum dimension for the large voids of $D_{\min }=50 \mathrm{~h}^{-1}$ $\mathrm{Mpc}$, we have processed the 8 observational samples with both search methods ( $p$ and $\nu$ ) and with all three grouping options (compact, medium-compact, loose), producing in this way 48 void catalogues. However, in this paper only the 16 catalogues corresponding to the medium-compact grouping option have been used to analyse further the void properties.

The generated void catalogues, compared to similar wide-field studies, contain a larger number of voids out to larger distances, described by more parameters (e.g. equivalent diameter, total volume, sphericity). They can be used as identification lists of the voids of clusters in the Northern Galactic Hemisphere, for studies of individual voids, as well as for statistical studies.

The examination of visualizations of the 3-D distribution of the voids from different void catalogues suggests a void-filled Universe with closely packed and intersecting voids. 2-D cross-sections of the 3-D distribution, however, indicate that the large voids may be separated by large zones of enhanced density of the tracing objects.

The automated comparison of the void catalogues corresponding to tracers of different richness class has shown that most of the voids in the distribution of the $R \geq 1$ $\mathrm{A} / \mathrm{ACO}$ clusters can be identified in the distribution of the $R \geq 0$ clusters.

The comparison of our void catalogues with compilations of voids known from other studies has shown that about $20 \%$ of the voids in the nearer and more complete volume $V 1(z \leq 0.09)$ and $90 \%$ of the more distant voids are newly discovered voids or void candidates. Their real- ity must be confirmed by future complete redshift surveys of clusters of galaxies.

The statistical significance of the voids of $R \geq 1$ A/ACO clusters has been verified by a two-sample Kolmogorov-Smirnov test. The results from the test reject the hypothesis that the large voids of rich clusters are random fluctuations.

We have processed statistically the void catalogues in order to derive mean characteristics (mean and median values) for the void dimensions, volumes, and sphericities. Analyzing the dependence of the diameters of the void constituent spheres on distance and on galactic latitude, we have found that homogeneous samples of voids of $R \geq$ $1 \mathrm{~A} / \mathrm{ACO}$ clusters not affected significantly by the observational selection should be extracted from a volume limited by $b \geq+50^{\circ}$ and $z \leq 0.09$. We suggest that our estimates of the equivalent void diameters of the $R \geq 1$ and $R \geq 0 \mathrm{~A} / \mathrm{ACO}$ clusters $D_{\mathrm{e}}=105.0 \pm 5.6 h^{-1} \mathrm{Mpc}$, and $D_{\mathrm{e}}=87.2 \pm 4.1 h^{-1} \mathrm{Mpc}$, respectively, are improved estimates of the dimensions of large voids. We have obtained for the first time mean estimates of the volumes and sphericities of the voids of clusters. The high value of the mean sphericity $(\sim 0.9)$ shows that the voids of clusters have quite spherical shapes.

Jointly processing the void catalogues and samples of objects of different type, we have identified the populations of clusters, groups, and galaxies in the voids of $R \geq 1$ $\mathrm{A} / \mathrm{ACO}$ clusters, as well as in the void shells. The estimates of the mean number densities of the void populations show that the voids of $R \geq 1 \mathrm{~A} / \mathrm{ACO}$ clusters are also underdense regions in the distribution of the poorer clusters and groups, and in the distribution of galaxies. We have constructed radial density profiles of the voids of $R \geq 1 \mathrm{~A} / \mathrm{ACO}$ clusters which suggest that they contain smaller voids of poorer clusters and groups, and still smaller voids of galaxies, in agreement with the concept for a void hierarchy.

Acknowledgements. The author is very grateful to David Batuski and his co-authors for providing the data of the MX Northern Abell Cluster Redshift Survey in advance of publication, as well as to Viktor Lebedev and Irina Lebedeva for the use of the updated version of their Compilation of Clusters of Galaxies with Published Redshifts. Thanks are due to Heinz Andernach for providing redshift data for the Abell/ACO clusters. His remarks on the redshifts used in this study were very helpful for improving the data. Thanks are due to Rudolf Dümmler and Gotthard Richter for useful comments on part of the results. The author wishes to thank Milcho Tsvetkov for the use of the computer complex of the Wide-Field Plate Database, as well as Katya Tsvetkova and Evgeni Semkov for help in the preparation of the manuscript. Thanks are due to the anonymous referee for a number of helpful suggestions. This research has made use of the NASA/IPAC extragalactic database (NED) which is operated by the Jet Propulsion Laboratory, California Institute of Technology, under contract with the National Aeronautics and Space Administration. 
The present study was partly supported by the National Science Fund of Bulgaria under Grant F-650/1996.

\section{References}

Abell G.O., 1958, ApJS 3, 211

Abell G.O., Corwin H.G.Jr., Olowin R.P., 1989, ApJS 70, 1 (ACO)

Aikio J., Mähönen P., 1998, ApJ 497, 534

Albert C.E., White R.A., Morgan W.W., 1977, ApJ 211, 309

Andernach H., 1997 (private communication)

Andernach H., Tago E., 1998, in: Müller V. et al. (eds.) Large Scale Structure: Tracks and Traces. World Scientific Press, Singapore, p. 147

Bahcall N.A., Cen R., 1993, ApJ 407, L49

Bahcall N.A., Soneira R.M., 1982a, ApJ 258, L17

Bahcall N.A., Soneira R.M., 1982b, ApJ 262, 419

Batuski D.J., Burns J.O., 1985, AJ 90, 1413

Batuski D.J., Burns J.O., Newberry M.V., Hill J.M., Deeg H.J., Laubscher B.E., Elston R.J., 1991, AJ 101, 1983

Batuski D., Slinglend K., Hill J.M., Haase S., Miller C., Michaud K., 1998, in: McLean B. et al. (eds.) Proc. IAU Symp. 179, New Horizons from Multiwavelength Sky Surveys. Kluwer, Dordrecht, p. 344

Broadhurst T.J., Ellis R.S., Koo D.C., Szalay A.S., 1990, Nat 343, 726

Burns J.O., Moody J.W., Brodie J.P., Batuski D.J., 1988, ApJ 335,542

Ciardullo R., Ford H., Bartko F., Harms R., 1983, ApJ 273, 24

Cruzen S.T., Weistrop D., Hoopes C.G., 1997, AJ 113, 1983

de Lapparent V., Geller M.J., Huchra J.P., 1991, ApJ 369, 273

Dey A., Strauss M.A., Huchra J., 1990, AJ 99, 463

Doroshkevich A.G., et al., 1996, MNRAS 283, 1281

Dubinski J., da Costa N., Goldwirth D.S., Lecar M., Piran T., 1993, ApJ 410, 458

Einasto J., Corwin H.G., Huchra J., Miller R.H., Tarenghi M., 1983, in: West R. (ed.) Highlights of Astronomy. Reidel, Dordrecht, Vol. 6, p. 757

Einasto J., Einasto M., Gramann M., 1989, MNRAS 238, 155

Einasto M., Einasto J., Tago E., Dalton G.B., Andernach H., 1994, MNRAS 269, 301

Einasto M., Tago E., Jaaniste J., Einasto J., Andernach H., 1997, A\&AS 123, 119

El-Ad H., Piran T., 1997, ApJ 491, 421

El-Ad H., Piran T., da Costa L.N., 1996, ApJ 462, L13

El-Ad H., Piran T., da Costa L.N., 1997, MNRAS 287, 790

Frisch P., Einasto J., Einasto M., Freudling W., Fricke K.J., Gramann M., Saar V., Toomet O., 1995, A\&A 296, 611

Geller M.J., Huchra J.P., 1983, ApJS 52, 61

Gioia I.M., Maccacaro T., Schild R.E., Wolter A., Stocke J.T., Morris S.I., Henry J.P., 1990, ApJS 72, 567

Gregory S.A., Thompson L.A., 1978, ApJ 222, 784

Gunn J.E., Hoessel J.G., Oke J.B., 1986, ApJ 306, 30

Hickson P., 1982, ApJ 255, 382

Hopp U., Materne J., 1985, A\&AS 61, 93

Huchra J.P., Geller M.J., 1982, ApJ 257, 423

Huchra J.P., Geller M.J., Clemens C.M., Tokarz S.P., Michel A., 1992, Bull. Inf. CDS 41, 31

Jõeveer M., Einasto J., 1978, in: Longair M.S. and Einasto J. (eds.) Proc. IAU Symp. 79, The Large Scale Structure of the Universe. Reidel, Dordrecht, p. 241
Kalinkov M., Stavrev K., Kuneva I., 1985, Astron. Nachr. 306, 283

Kirshner R.P., Oemler A. Jr., Schechter P.L., Shectman S.A., 1981, ApJ 248, L57

Lebedev V.S., Lebedeva I.A., 1991, Astrophys. Invest. 31, 91

Ledlow M.J., Loken C., Burns J.O., Hill J.M., White R.A., 1996, AJ 112, 388

Lin H., Kirshner R.P., Shectman S.A., Landy S.D., Oemler A., Tucker D.L., Schechter P.L., 1996, ApJ 464, 60

Lindner U., Einasto J., Einasto M., Freudling W., Fricke K., Tago E., 1995, A\&A 301, 329

Lindner U., Einasto M., Einasto J., Freudling W., Fricke K., Lipovetsky V., Pustilnik S., Izotov Y., Richter G., 1996, A\&A 314,1

Madore B.F., Helou G., Corwin H.G., Schmitz M., Wu X., Bennett J., 1992, ASP Conf. Ser. 25, 47

Morgan W.W., Kayser S., White R.A., 1975, ApJ 199, 545

Noonan T.W., 1973, AJ 78, 26

Otto S., Politzer H.D., Preskill J., Wise M.B., 1986, ApJ 304, 62

Peacock J.A., West M.J., 1992, MNRAS 259, 494

Plionis M., Valdarnini R., Jing Y.P., 1992, ApJ 398, 12

Ramella M., Geller M.J., Huchra J.P., 1989, ApJ 344, 57

Ryden B.S., Turner E.L., 1984, ApJ 287, L59

Sage L.J., Weistrop D., Cruzen S., Kömpe C., 1997, AJ 114, 1753

Scaramella R., Zamorani G., Vettolani G., Chincarini G., 1991, AJ 101, 342

Shectman S.A., 1985, ApJS 57, 77

Slinglend K., Batuski D., Miller C., Haase S., Michaud K., Hill J.M., 1998, ApJS 115, 1

Stavrev K.Y., 1990a, Publ. Astron. Dept. Eötvös Univ. 10, 115

Stavrev K.Y., 1990b, in: Jaschek C. and Murtagh F. (eds.) Errors, Bias and Uncertainties in Astronomy. Cambridge Univ. Press, Cambridge, p. 411

Stavrev K.Y., 1990c, in: 12th European Regional Astronomy Meeting of the IAU, Davos, Switzerland, Abstract Book, p. V-17

Stavrev K.Y., 1991, Institute of Math. Statistics Bull. 20, 275

Stavrev K.Y., 1998, in: McLean B. et al. (eds.) Proc. IAU Symp. 179, New Horizons from Multiwavelength Sky Surveys. Kluwer, Dordrecht, p. 332

Szomoru A., van Gorkom J.H., Gregg M.D., Strauss M.A., 1996, AJ 111, 2150

Tifft W.G., Gregory S.A., 1976, ApJ 205, 696

Tifft W.G., Gregory S.A., 1988, AJ 95, 651

Tully R.B., 1986, ApJ 303, 25

Tully R.B., 1987, ApJ 323, 1

Voges W., 1992, in: MacGillivray H.T. and Thomson E.B. (eds.) Digitised Optical Sky Surveys. Kluwer, Dordrecht, p. 453

Weistrop D., Hintzen P., Liu C., Lowenthal J., Cheng K.-P., Oliversen R., Brown L., Woodgate B., 1995, AJ 109, 981 van de Weigaert R., van Kampen E., 1993, MNRAS 263, 481 Willick J.A., Brodie J.P., Bowyer S., 1990, ApJ 355, 393

Zwicky F., Herzog E., Wild P., Karpowicz M., Cowal C., 1961-1968, Catalog of Galaxies and Clusters of Galaxies, Vols. 1-6, California Institute of Technology, Pasadena 